\begin{tabular}{|c|c|c|}
\hline \multirow{3}{*}{$\begin{array}{l}\text { ITC 4/46 } \\
\text { Journal of Information Technology } \\
\text { and Control } \\
\text { Vol. 46/ No. 4 / 2017 } \\
\text { pp. 566-604 } \\
\text { DOI 10.5755/j01.itc.46.4.18215 } \\
\text { ๑ Kaunas University of Technology }\end{array}$} & \multicolumn{2}{|c|}{$\begin{array}{l}\text { On the Importance of the Artificial Bee Colony } \\
\text { Control Parameter 'Limit' }\end{array}$} \\
\hline & Received 2017/05/21 & Accepted after revision $2017 / 10 / 23$ \\
\hline & \multicolumn{2}{|c|}{ rrossef http://dx.doi.org/10.5755/j01.itc.46.4.18215 } \\
\hline
\end{tabular}

\title{
On the Importance of \\ the Artificial Bee Colony Control Parameter 'Limit'
}

\section{Niki Veček}

University of Maribor, Faculty of Electrical Engineering and Computer Science, Koroška cesta 46, 2000 Maribor, Slovenia, e-mail: niki.vecek@gmail.com

\section{Shih-Hsi Liu}

California State University, Fresno, Department of Computer Science, 2576 E San Ramon Ave., Fresno, CA, USA, e-mail:shliu@mail.fresnostate.edu

\section{Matej Črepinšek, Marjan Mernik}

University of Maribor, Faculty of Electrical Engineering and Computer Science, Koroška cesta 46, 2000 Maribor, Slovenia, e-mail:matej.crepinsek@um.si,marjan.mernik@um.si

Corresponding author: niki.vecek@gmail.com

Artificial Bee Colony (ABC) is a successful meta-heuristic algorithm that has been greatly utilised by researchers. Through our practical experience of $A B C$, we have noticed that the recommended formula 'limit' $=n_{e}{ }^{*} D$ may not be the best choice for different problems. In this work, a set of experiments using horizontal and vertical approaches has been designed and executed with the aim of observing the effect of 'limit' on ABC. The results have been statistical analysed using Null Hypothesis Significance Testing (NHST) as well as the Chess Rating System for Evolutionary Algorithms (CRS4EAs), which is a novel approach for comparing meta-heuristic algorithms. It is shown that the recommended formula is not the best setting for different problems and approaches. Hence, the control parameter 'limit' should be tuned or controlled. The other important result of this study is to show that CRS4EAs is comparable but also shows benefits over NHST.

KEYWORDS: ABC, control parameter setting, sensitivity analysis, significance testing, chess rating system for evolutionary algorithms. 


\section{Introduction}

Comparisons between different meta-heuristic algorithms [5] are inevitably necessary within the field of Evolutionary Computation (EC). Although the scientific testing [20] approach, the aim of which is to learn about which kinds of problems and why one algorithm performs better, is preferred over the horse racing approach [12], [23], [45], the aim of which is to outperform other algorithms, the latter approach still prevails during current EC experimental practices. However, even in the scientific testing approach, simply understanding parameter interactions and placing emphasis on the analysis of robustness may not be enough if an algorithm under investigation performs badly. Hence, there is still a need for comparing the performances of the algorithms under investigation using the currently best available algorithms [9].

This paper deals with the Artificial Bee Colony (ABC) algorithm [25], [26], [39], which is a swarm intelligence algorithm that accomplishes optimisation tasks through social cooperation among bees (i.e., individuals) - employed bees exploit food source and share food source information to onlooker bees; onlooker bees probabilistically choose and exploit food source based on the provided information; and scout bees explore new food source when current ones are exhausted. ABC exhibits remarkable balance between exploitation and exploration [8] (raw data for experiments presented in this paper are available in [47]). This balance between exploitation (employed bee phase and onlooker bee phase) and exploration (scout bee phase) is controlled by population size $(S N)$ and 'limit', respectively. The formula 'limit' $=n_{e}{ }^{*} D$ ('limit' is the threshold for determining whether a scout bee should be introduced or not, $n_{e}$ is the number of employed bees, $D$ is the dimension of a problem) was recommended in a very influential paper [26]. As $A B C$ is a very successful algorithm, it has been used extensively over recent years [28], [29]. The suggested formula for setting the 'limit' control parameter is indeed mostly used (e.g., [2]). We came across only a few studies where the 'limit' was set at a certain fixed number (e.g., 10 in [38], [55], 30 in [53], 40 in [56], 50 in [44], 100 in [22], [52], 200 in [34], [57]), or better where the 'limit' was tuned [35]. When experimenting using $A B C$ we have noticed its sensitiveness to 'limit' control parameter and that its relationship between population size $\left(S N=2 * n_{e}\right)$ and the dimension of a problem $(D)$ is not straightforward. However, this was just our speculation driven by practical experience with ABC. Hence, we decided to perform extensive statistical analysis of $A B C$ and support it by stronger conclusions, using the Null Hypothesis Significance Testing (NHST) [41] and Chess Rating System for Evolutionary Algorithms (CRS4EAs) [48]. For finding the significant differences with NHST, the Wilcoxon's test [51] was a more appropriate test with the post-hoc analysis supported by the Holm's test [19]. Both the Wilcoxon's test and CRS4EAs compare the results pairwisely but whilst the Wilcoxon's comparison concentrates only on $1 \times k$ comparison, the comparison in CRS4EAs allows $k \times k$ comparison and the detections of significant differences amongst all algorithms. Note that when attempting to apply statistical $k \times k$ comparison, the more appropriate test would be the Friedman test [13], [14]. However, as the number of problems is really small and the goal was to analyse different 'limit' settings regarding different problems, the Friedman test could not be taken into consideration [50]. Hence, the choice of Wilcoxon's test with post-hoc Holm's test is shown as an appropriate one. Even though CRS4EAs allows $k \times k$ comparison and Wilcoxon's test allows only $1 \times k$ comparison, the analysis of CRS4EAs was applied as $1 \times k$ comparison, as well as assuring that both methods are applied equally. Our results show that ABC's performance is very sensitive to a control parameter 'limit', which is often independent regarding the population size. Whilst the 'limit' depends on dimension $D$, it is much more dependent on the problem under investigation. Although, the characteristics of a problem might drastically change when changing dimension $D$ and can become a completely different problem (e.g., an optimisation function becomes multi-modal instead of uni-modal or vice verse, and the fitness-distance correlation is changed from high to low correlation or vice verse [7]). Hence, dimension $D$ can be seen as part of a problem as well.

The main contributions of this paper are:

- Sensitivity analysis is applied for the first time on $A B C$ control parameters using vertical and horizontal approaches showing that control parameter $S N$ is much more robust than control 
parameter 'limit', which must be carefully set for the best results;

_ An example of how from sensitivity analysis one might conclude that a suggested formula for setting control parameters is not most appropriate; Deep statistical investigations about setting ABC control parameter 'limit' as a full factorial design using NHST and CRS4EAs showing that the recommended formula for setting the control parameter 'limit' regarding population size $S N$ and the dimension of the problem $D$ is not the best for every problem and approach;

- For the first time, it is shown that even the control parameter 'limit' depends on the available maximum number of fitness evaluations, and that $\mathrm{ABC}$ convergence using the suggested formula is not amongst the fastest; and

_ First application of CRS4EAs as $1 \times k$ comparison showing its applicability and suitability as a feasible replacement of NHST.

The main conclusion from this study is that $A B C$ does not always perform best when under the setting 'limit' $=n_{e}{ }^{*} \mathrm{D}$. Hence, the 'limit' control parameter should be tuned or controlled.

However, such a conclusion should not come as a surprise in EC and confirms already established knowledge within the meta-heuristic field. Namely, fixed formulae for setting a control parameter usually lead to poor performances when applying to different problems. However, a systematic mapping study from [39] shows that this formula is indeed very frequently used indicating that still many researchers believe that some fixed formulae can be a robust choice. Our speculation is that this dichotomy between theory and practice exists due to lack of $A B C$ studies showing that such a parameter setting is not the best. In this respect, our work can be seen as remedying this situation for $A B C$. There should be no excuse not to perform tuning on control parameter 'limit' anymore. The other important conclusion from this study is that CRS4EAs is comparable with NHST but CRS4EAs also showed many benefits during experimentation where a greater number of experiments needed to be conducted. When executing one tournament in CRS4EAs, all the necessary data for analysis are obtained and calculated, whilst for NHST there are always additional tests required. Having so many different situations and approaches, the results analysed by CRS4EAs are far quicker and easier than with NHST.

The paper is organized as follows. Section 2 describes the conducted experiment in detail. This section is divided into three major parts: in Section 2.1 the sensitivity analysis is conducted for one optimisation problem; in Section 2.2 the results of experiment are analysed with NHST and the results reported regarding the different approaches; in Section 2.3 the results of the experiment are analysed with CRS4EAs and results are again reported regarding the different approaches. Section 3 displays the results of tuning the parameters of $A B C$ on different dimensionalities of one optimisation problem. Section 4 discusses other similar researches as presented in the past. Lastly, Section 5 concludes the paper. All the algorithms, figures and tables are also placed online at https://lpm. feri.um.si/research/abc/.

\section{Experiment}

The amount of exploration [8] of $\mathrm{ABC}$ is controlled by the control parameter 'limit'. ABC is exploring the search space more often when the 'limit' is set at a small number, and vice versa by exploiting the search space when the 'limit' is set to a higher number (Algorithm 1). The amount of exploration and exploitation depends on the problem and even on the evolution stage [8]. Hence, it is difficult to quantify. The formula 'limit' = $n_{e}{ }^{*} \mathrm{D}[26]$ suggests that higher-dimensional problems require less exploration (higher dimension increases 'limit', which in turn decreases exploration), and that bigger population size increases exploitation, which is indeed correct for ABC. However, the relationship between population size and the needed amount of exploration is unclear, as well as the fact that higher-dimensional problems might require more exploration. Overall, the suggested formula was not intuitive for us and we decided to further explore the relationships between population size $S N\left(S N=2 * n_{e}\right)$, dimension $D$, and control parameter 'limit'. Our experiment was divided into two parts. In the first part, the importance of $S N, D$, and 'limit' to ABC was investigated by performing sensitivity analysis [33], which showed that indeed the most influential one amongst the aforementioned factors is 'limit'. In the second part of the experiment, 
emphasis was given to the $\mathrm{ABC}$ control parameter 'limit', where different settings were statistically analysed by NHST and CRS4EAs.

During the experiment, we used the same benchmark functions as in the original ABC work [26]. Although this benchmark suite contained only five numerical benchmark functions: (1) multi-modal, non-separable Schaffer function $f_{1}$, (2) uni-modal, separable Sphere function $f_{2}$, (3) multi-modal, non-separable Griewank function $f_{3}$, (4) multi-modal, separable Rastrigin function $f_{4}$, and (5) uni-modal, non-separable Rosenbrock function $f_{5}$, it was enough to arrive at appropriate conclusions. Even this small benchmark suite confirmed our hypothesis and there was no need to perform the experiment on more comprehensive benchmarks. On the other hand, whenever a statistical formula is suggested, it should be tested on comprehensive sets of benchmarks that can really support it on a vast number of different optimisation problems. For example, Piotrowski in [43] suggested that both the problems, minimisation and maximisation should be used on the same benchmark functions since a good performance of a meta-heuristic algorithm on the minimisation of some function does not also guarantee a good performance on the maximisation of the same function, and vice versa.

We extended Karaboga's experiment [26] by performing a full factorial design on this benchmark suite using the following factors and their values: $S N=\{24,50,100\}$, $D=\{2,5,10,30,50\}$, and 'limit' $=\{0,100,250,500,750$, $1000,1250,1500, \infty\}$. Hence, altogether there were $3 * 5$ * $9=135$ different combinations tested using 100 independent runs, whilst using both vertical and horizontal approaches [18] when performing the experiments.

In the first case, known also as 'the fixed-cost approach', we measured the quality of a solution reached by a pre-defined number of fitness evaluations $(100,000$ and 250,000 fitness evaluations for each combination). In the second case, also known as 'the fixed-target approach', we measured the number of fitness evaluations needed to find a (sub-)optimal solution $\left(10^{-6}\right.$ and $10^{-12}$ ). The horizontal approach would have stopped the algorithm if a (sub-)optimal solution could not be found over 1,000,000 fitness evaluations.

\subsection{Sensitivity Analysis}

In this subsection, the results of the first part of the experiment are presented showing the importance of $S N, D$, and 'limit' to the performance of ABC. Sensitiv- ity analysis [33] is shown only for $f_{1}$ due to its similarity of results on $f_{2}-f_{5}$. The other reason is that the emphasis of this study was given to the second part of the experiment, where different settings of 'limit' were statistically analysed, and in the third part where the results were analysed using a novel method for pairwise comparison, CRS4EAs.

The aim of sensitivity analysis was to show the robustness of a meta-heuristic algorithm against different settings of control parameters. By performing a sensitivity analysis, we could find those control parameters (if any) that are very sensitive, as well of those (if any) which are very robust. In the former case, a proper setting of a control parameter is crucial for obtaining good performance of a meta-heuristic algorithm, whilst in the latter case, similar performance can be achieved regardless of the different settings of such non-sensitive control parameters. An obvious question may arise as to why the dimensionality of problem $D$ was included within our sensitivity analysis as a factor as it is not a control parameter but $D$ should be considered as part of the optimisation problem? As the formula 'limit' $=n_{e}{ }^{*} D[26]$ suggested a particular correlation between 'limit' and two other variables: population size and dimensionality of a problem, such a correlation should probably be indicated by sensitivity analysis as well. If at least one of these factors is insensitive, then the suggested formula [26] might not capture the relationships amongst the factors too well. As shown in the continuation, this was indeed the case.

In Tables 1(a) and 1(b), the experimental results of $f_{1}$ are presented when using the vertical approach with 100,000 and 250,000 maximum number of fitness evaluations (MaxFEs in the tables appeared later), respectively. In Tables 2 (a) and 2(b), the experimental results of $f_{1}$ are presented when using the horizontal approach in order to find a (sub-)optimal solution at $10^{-6}$ and at $10^{-12}$, respectively. The best results are highlighted by a light grey colour.

The difference between Tables 1(a) and 1(b) shows that 250,000 fitness evaluations were almost always enough for $f_{1}$ to find the exact solution; except for high dimensions $D=30$ and $D=50$, or when 'limit' $=0$ (high exploration) and 'limit' $=\infty$ (no exploration). The Karaboga's setting of 'limit' $L_{k}$ was always the better performer regarding the mean value when 250,000 fitness evaluations were available (Table 1(b)), whilst when only 100,000 were available (Table $1(a)$ ), the 
Algoritm 1: The pseudo-code of algorithm $A B C$

Data: Set the control parameters of the $\mathrm{ABC}$ algorithm

$S N$ : Population size

limit: Maximum number of trials for abandoning a source

$M F E$ : Maximum number of fitness evaluations

begin

//Initialization;

num_eval $\longleftarrow 0$;

for $s=1$ to $S N$ do

$X(s) \longleftarrow$ random solution by Eq. 1 [26];

$f_{s} \longleftarrow f(X(s))$;

trial $(s) \longleftarrow 0$;
num_eval ++ ;

end

repeat

//Employed Bees Phase;

for $s=1$ to $S N$ do

$x^{\prime} \longleftarrow$ a new solution produced by Eq. 2 [26];

$f\left(x^{\prime}\right) \longleftarrow$ evaluate new solution;

num_eval ++ ;

if $f\left(x^{\prime}\right)<f_{s}$ then

$X(s) \longleftarrow x^{\prime} ; f_{s} \longleftarrow f\left(x^{\prime}\right) ; \operatorname{trial}(s) \longleftarrow 0 ;$

else

$\mid \operatorname{trial}(s) \leftarrow \operatorname{trial}(s)+1$;

end

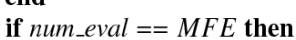

| Memorize the best solution achieved so far and exit main repeat; end

end

Calculate the probability values $p_{i}$ for the solutions using fitness values by Eqs. 3 and 4 [26];

//Onlooker bee phase;

$s \longleftarrow 1 ; t \longleftarrow 1$;

repeat

$r \longleftarrow \operatorname{rand}(0,1)$

if $r<p(s)$ then

$t \longleftarrow t+1$

$x^{\prime} \longleftarrow$ a new solution produced by Eq. $2[26]$;

$f\left(x^{\prime}\right) \longleftarrow$ evaluate new solution;

num_eval ++ ;

if $f\left(x^{\prime}\right)<f_{s}$ then

$X(s) \longleftarrow x^{\prime} ; f_{s} \longleftarrow f\left(x^{\prime}\right) ; \operatorname{trial}(s) \longleftarrow 0 ;$

else

$\operatorname{trial}(s) \longleftarrow \operatorname{trial}(s)+1$;

end

if num_eval $==M F E$ then

Memorize the best solution achieved so far and exit main repeat; end

end

$s \longleftarrow(s \bmod S N)+1$

until $t=S N$;

//Scout Bee Phase;

$m i \longleftarrow\{s: \operatorname{trial}(s)=\max ($ trial $)\}$

if $\operatorname{trial}(m i)>=$ limit then

$X(m i) \longleftarrow$ random solution by Eq. $1[26]$;

$f_{m i} \longleftarrow f(X(m i))$;

num_eval ++;

trial $($ mi $) \longleftarrow 0$;

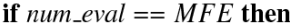

| Memorize the best solution achieved so far and exit main repeat;

end

Memorize the best solution achieved so far;

until num_eval $=M F E$; 
Table 1

Mean values (Mean) and standard deviation values (SD) for the vertical approach to problem $f_{1}$

\begin{tabular}{|c|c|c|c|c|c|c|c|c|c|c|c|}
\hline & & $L_{K}$ & $L_{0}$ & $L_{100}$ & $I_{250}$ & $L_{500}$ & $I_{750}$ & $L_{1000}$ & $L_{1250}$ & $L_{1 !}$ & $T_{x_{x}}$ \\
\hline $\mathrm{N}=24$ & Mean & $00 \mathrm{E}+00$ & $2.04 \mathrm{E}-15$ & $0.00 \mathrm{E}+00$ & $0.00 \mathrm{E}+00$ & 5E-19 & $0.00 \mathrm{E}+1$ & $5 \mathrm{E}-$ & $1.67 \mathrm{E}-18$ & $22 \mathrm{E}-1$ & $\overline{0}$ \\
\hline$=2$ & SD & 00 & $5.26 \mathrm{E}-15$ & $0.00 \mathrm{E}+00$ & $0.00 \mathrm{E}+00$ & $2 \mathrm{E}-18$ & $0.00 \mathrm{~F}$ & & $9.47 \mathrm{E}$ & $1.09 \mathrm{~F}$ & $1.42 \mathrm{E}-17$ \\
\hline $\mathrm{v}=24$ & Mean & $8 \mathrm{E}-14$ & $7.39 \mathrm{E}-06$ & $0.00 \mathrm{E}+00$ & $0.00 \mathrm{E}+00$ & 5E-19 & $0.00 \mathrm{E}+$ & $.35 \mathrm{E}-18$ & $1.67 \mathrm{E}-18$ & $2.22 \mathrm{E}-18$ & $.11 \mathrm{E}-18$ \\
\hline$=5$ & SD & $9 \mathrm{E}-13$ & $9.29 \mathrm{E}-06$ & $0.00 \mathrm{E}+00$ & $0.00 \mathrm{E}+00$ & 52E-18 & & & $47 \mathrm{E}$ & $1.09 \mathrm{I}$ & $4.50 \mathrm{E}-17$ \\
\hline 24 & Mean & $22 \mathrm{E}-10$ & (0E-04 & 3E- & $0.00 \mathrm{E}$ & $3.01 \mathrm{~F}$ & $5.00 \mathrm{E}$ & $4.61 \mathrm{E}-$ & OE. & .15 & $17 \mathrm{E}-1$ \\
\hline 10 & SD & $99 \mathrm{E}-199$ & $6.51 \mathrm{E}-(2)$ & $1.14 \mathrm{E}-06$ & $0,00 \mathrm{E}+00$ & $47 \mathrm{E}-15$ & $4 . / 0 t$ & $5.10 \mathrm{E}-15$ & $1.61 \mathrm{E}-13$ & $1.04 \mathrm{E}-12$ & $1.16 \mathrm{E}-11$ \\
\hline$=24$ & Mean & $9 \mathrm{E}-06$ & $1.49 \mathrm{E}-02$ & $4.84 \mathrm{E}-103$ & 04 & $2.76 \mathrm{E}-06$ & $1.99 \mathrm{~F}$ & .15E-16 & 1.11E-06 & $1.43 \mathrm{E}-06$ & $4.89 \mathrm{E}-0 \mathrm{t}$ \\
\hline$=30$ & SD & $9 \mathrm{E}-05$ & $1.81 \mathrm{E}-02$ & $5.66 \mathrm{E}-03$ & $2.45 \mathrm{~F}$ & $8.12 \mathrm{E}-06$ & 1. & $3.00 \mathrm{~F}$ & 3.23E- -06 & $3,46 \mathrm{E}-1160$ & $42 \mathrm{E}-$ \\
\hline$=24$ & Mean & $5 E-04$ & 3.7 & $2.44 \mathrm{I}$ & $4.68 \mathrm{E}-03$ & 04 & 1.2 & 1.000 & $9.53 \mathrm{E}-105$ & 1.2 & $9.00 \mathrm{E}-0$ \\
\hline 50 & SD & 04 & 4.4 & $2.76 \mathrm{E}$ & $9.23 \mathrm{E}-03$ & 04 & 3.7 & & $2.43 \mathrm{E}-104$ & $3.22 \mathrm{E}-04$ & $2.11 \mathrm{E}-0$ \\
\hline $\mathrm{J}=50$ & Mean & +00 & $2.62 \mathrm{E}-15$ & $0.00 \mathrm{E}+00$ & $0.00 \mathrm{E}+00$ & $0.00 \mathrm{E}+00$ & 0.001 & $5 . .5 \mathrm{~F}$ & $1.67 \mathrm{t}$ & $0.00 \mathrm{E}+00$ & -18 \\
\hline 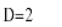 & SD & $\mathbb{E}+1$ & $8.06 \mathrm{E}-15$ & $0.00 \mathrm{E}+00$ & $0.00 \mathrm{E}+00$ & $0.00 \mathrm{E}+00$ & 0.00 & & $9.47 \mathrm{E}-18$ & $0.00 \mathrm{E}$ & 18 \\
\hline$=50$ & Mean & & 6.20 & $\mathrm{E}+00$ & 00 & & & & & & E-18 \\
\hline & & & 6.9 & 00 & & 18 & & 7.7 & 18 & 1.32 & $1.42 \mathrm{E}-17$ \\
\hline 50 & Mean & 8 & $5.01 \mathrm{E}$ & $64 \mathrm{E}-07$ & & & & & 1.7 & 1.39 & $82 \mathrm{E}-13$ \\
\hline 10 & SD & 7.7 & 04 & 7E-07 & & & & & $1.51 \mathrm{~F}$ & 1.36 & $8.64 \mathrm{E}-12$ \\
\hline$=50$ & Mean & $1.43 \mathrm{E}-06$ & $1.59 \mathrm{E}-02$ & $5.94 \mathrm{E}-03$ & $9.03 \mathrm{E}-05$ & 06 & 1.4 & 1.8 & 7.94 & 8.64 & $2.99 \mathrm{E}-0$ \\
\hline 30 & SD & $8 \mathrm{E}-06$ & $1.51 \mathrm{IE}-02$ & -03 & 2.33E-04 & .06 & $5.4 i$ & 7.02 & 1.94 & 2.39 & $1.84 \mathrm{E}-0$. \\
\hline $\mathrm{J}=50$ & Mean & 04 & $3.05 \mathrm{E}-02$ & $2.59 \mathrm{E}-02$ & $4,43 \mathrm{E}-03$ & $3.19 \mathrm{E}-04$ & 2.28 & $1.16 \mathrm{E}-04$ & 1.11 & 1.86 & $1.32 \mathrm{E}-0$ \\
\hline$=50$ & SD & 7E-04 & 3. & $1 \mathrm{E}-02$ & 6.48 & $56 \mathrm{E}-04$ & 8.67 & $2.80 \mathrm{E}-04$ & $2.57 \mathrm{E}-0$ & $7.94 \mathrm{E}-04$ & $4.33 \mathrm{E}-04$ \\
\hline$V=100$ & Mean & $00 \mathrm{E}+00$ & $5.90 \mathrm{E}-15$ & $0.00 \mathrm{E}+00$ & $0.00 \mathrm{E}+00$ & $0.00 \mathrm{E}+00$ & 0. & $.11 \mathrm{E}-18$ & $2.22 \mathrm{E}-18$ & $1.67 \mathrm{E}-18$ & 22E-18 \\
\hline$=2$ & SD & $0 \mathrm{E}+00$ & $2.55 \mathrm{E}-14$ & $0.00 \mathrm{E}+00$ & $0.00 \mathrm{E}+00$ & $0.00 \mathrm{E}+00$ & 0.001 & 7.77 & $1.09 \mathrm{E}-17$ & $9.47 \mathrm{E}-18$ & $19 \mathrm{E}-1$ \\
\hline $\mathrm{J}=100$ & Mean & 19 & $6.42 \mathrm{E}-06$ & +00 & & $0.00 \mathrm{~F}$ & & & 3.3 & $1.67 \mathrm{E}-18$ & $00 \mathrm{E}+$ \\
\hline$=$ & SD & $2 \mathrm{E}-18$ & $7.15 \mathrm{E}-06$ & $0.00 \mathrm{E}+00$ & $5.52 \mathrm{E}-18$ & $0.00 \mathrm{E}+00$ & 9.47 & $7.77 \mathrm{E}-18$ & $1.32 \mathrm{E}-17$ & 9.47E-18 & $0.00 \mathrm{E}+0$ \\
\hline $\mathrm{V}=100$ & Mean & $3 \mathrm{E}-17$ & $3.47 \mathrm{E}-04$ & $2.60 \mathrm{E}-07$ & $5.55 \mathrm{~F}$ & 3.83E-17 & 4.2 & 2.02 & $2.17 \mathrm{E}-14$ & $5.95 \mathrm{I}$ & 4.13E-1. \\
\hline$=10$ & SD & $5 \mathrm{E}-16$ & 4 & $9.61 \mathrm{E}-07$ & & & & & 1.461 & 5.88 & $2.61 \mathrm{E}$ \\
\hline$=100$ & Mea & $5-106$ & & 5.2 & & & & & $9.41 \mathrm{E}-0$ & $1.08 \mathrm{E}-06$ & $2.62 \mathrm{E}-0 \mathrm{t}$ \\
\hline 30 & SD & E-06 & 02 & -03 & & -05 & 4.67 & 4.13E-0. & $4.78 \mathrm{E}-06$ & $3.98 \mathrm{E}-06$ & $1.33 \mathrm{E}-0$. \\
\hline & & & & & & & & & $8.02 \mathrm{E}$ & $1.35 \mathrm{E}-04$ & $1.39 \mathrm{E}$ \\
\hline$=$ & SD & -04 & & -02 & & -04 & & & $E-1$ & 3.73E-04 & \\
\hline
\end{tabular}

(a) Vertical approach, $f_{1}$, MaxFEs $=100,000$

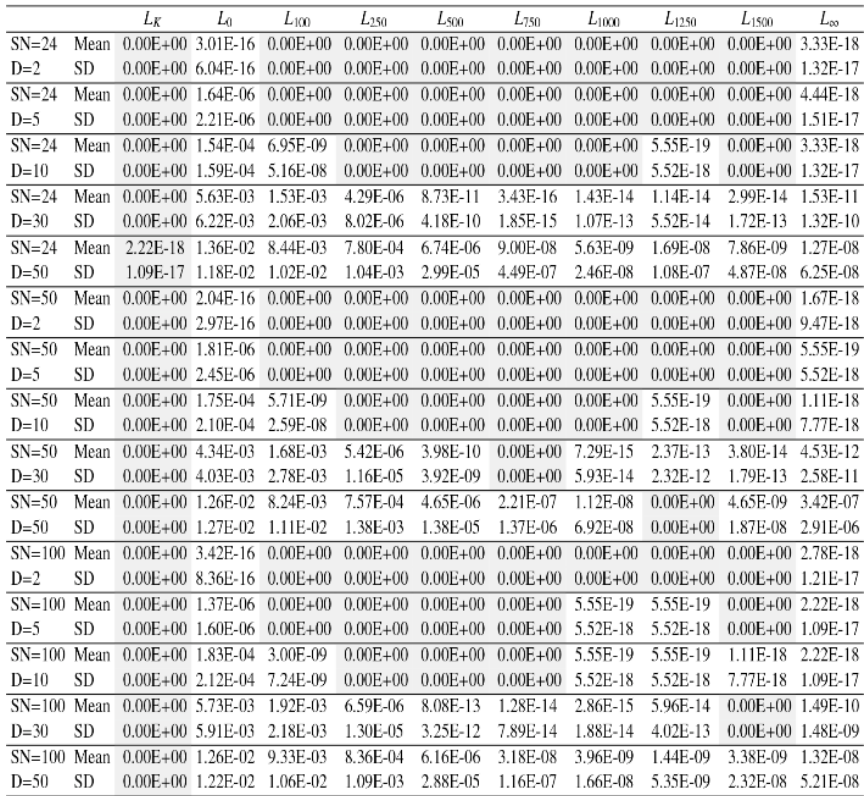

(b) Vertical approach, $f_{1}$, MaxFEs $=250,000$

\section{Table 2}

Mean values (Mean) and standard deviation values (SD) for the horizontal approach to $\operatorname{problem} f_{1}$

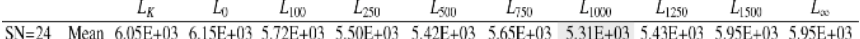

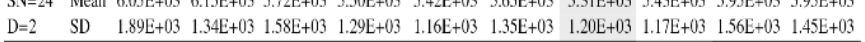

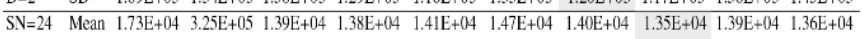
$\mathrm{D}=5 \quad \mathrm{SD} \quad 5.94 \mathrm{E}+03 \quad 3.00 \mathrm{E}+05 \quad 3.39 \mathrm{E}+03 \quad 3.39 \mathrm{E}+03 \quad 3.70 \mathrm{E}+03 \quad 3.66 \mathrm{E}+03 \quad 4.62 \mathrm{E}+03 \quad 3.73 \mathrm{E}+03 \quad 3.65 \mathrm{E}+03 \quad 3.26 \mathrm{E}+03$ $\begin{array}{llllllllllll}\mathrm{SN}=24 & \text { Mean } & 3.37 \mathrm{E}+04 & 9.80 \mathrm{E}+05 & 5.01 \mathrm{E}+04 & 2.70 \mathrm{E}+04 & 2.72 \mathrm{E}+04 & 2.70 \mathrm{E}+04 & 2.65 \mathrm{E}+04 & 2.72 \mathrm{E}+04 & 2.75 \mathrm{E}+04 & 2.75 \mathrm{E}+04\end{array}$

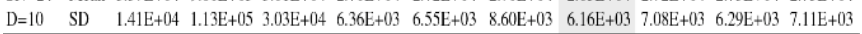
\begin{tabular}{llllllllllll}
\hline $\mathrm{SN}=24$ & Mean & $1.03 \mathrm{E}+05$ & $1.00 \mathrm{E}+06$ & $1.00 \mathrm{E}+06$ & $3.48 \mathrm{E}+05$ & $8.28 \mathrm{E}+04$ & $8.22 \mathrm{E}+04$ & $8.22 \mathrm{E}+04$ & $8.69 \mathrm{E}+04$ & $8.16 \mathrm{E}+04$ & $7.88 \mathrm{E}+04$
\end{tabular}

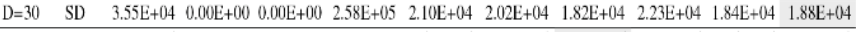
\begin{tabular}{llllllllllll}
\hline $\mathrm{SV}=24$ & Mean & $1.72 \mathrm{E}+05$ & $1.00 \mathrm{E}+06$ & $1.00 \mathrm{E}+06$ & $1.00 \mathrm{E}+06$ & $2.53 \mathrm{E}+05$ & $1.47 \mathrm{E}+05$ & $1.32 \mathrm{E}+05$ & $1.38 \mathrm{E}+05$ & $1.35 \mathrm{E}+05$ & $1.39 \mathrm{E}+05$
\end{tabular}

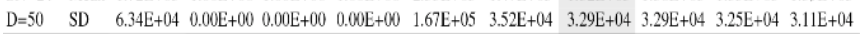

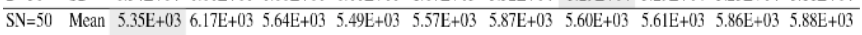

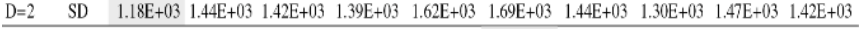
\begin{tabular}{llllllllllll}
\hline $\mathrm{SN}=50$ & Mean & $1.33 \mathrm{E}+04$ & $3.4 \mathrm{I} \mathrm{E}+05$ & $1.35 \mathrm{E}+04$ & $1.38 \mathrm{E}+04$ & $1.43 \mathrm{E}+04$ & $1.31 \mathrm{E}+04$ & $1.41 \mathrm{E}+04$ & $1.47 \mathrm{E}+04$ & $1.35 \mathrm{E}+04$ & $1.40 \mathrm{E}+04$
\end{tabular} \begin{tabular}{lllllllllllll}
$\mathrm{D}=5$ & $\mathrm{SD}$ & $3.14 \mathrm{E}+03$ & $3.15 \mathrm{E}+05$ & $3.57 \mathrm{E}+03$ & $3.10 \mathrm{E}+03$ & $3.29 \mathrm{E}+03$ & $3.05 \mathrm{E}+03$ & $4.11 \mathrm{E}+03$ & $3.92 \mathrm{E}+03$ & $3.15 \mathrm{E}+03$ & $3.53 \mathrm{E}+03$ \\
\hline
\end{tabular}

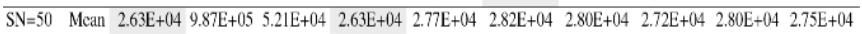

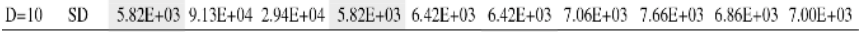
\begin{tabular}{lllllllllllll}
\hline $\mathrm{SV}=50$ & Mean & $7.89 \mathrm{E}+04$ & $1.00 \mathrm{E}+06$ & $9.90 \mathrm{E}+05$ & $4.08 \mathrm{E}+05$ & $8.48 \mathrm{E}+04$ & $7.89 \mathrm{E}+04$ & $8.17 \mathrm{E}+04$ & $8.10 \mathrm{E}+04$ & $8.26 \mathrm{E}+04$ & $7.95 \mathrm{E}+04$
\end{tabular}

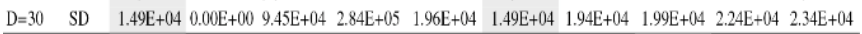

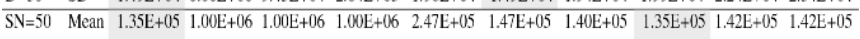

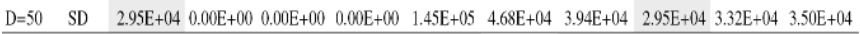

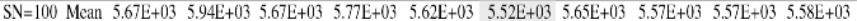

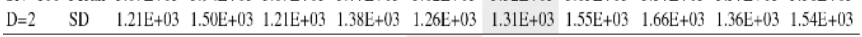
$\begin{array}{lllllllllllll}\mathrm{SN}=100 & \text { Mean } & 1.40 \mathrm{E}+04 & 2.93 \mathrm{E}+05 & 1.38 \mathrm{E}+04 & 1.40 \mathrm{E}+04 & 1.30 \mathrm{E}+04 & 1.40 \mathrm{E}+04 & 1.44 \mathrm{E}+04 & 1.38 \mathrm{E}+04 & 1.42 \mathrm{E}+04 & 1.37 \mathrm{E}+04\end{array}$ \begin{tabular}{llllllllllll}
$\mathrm{D}=5$ & $\mathrm{SD}$ & $3.80 \mathrm{E}+03$ & $2.80 \mathrm{E}+05$ & $3.29 \mathrm{E}+03$ & $3.80 \mathrm{E}+03$ & $2.83 \mathrm{E}+03$ & $3.45 \mathrm{E}+03$ & $3.43 \mathrm{E}+03$ & $3.29 \mathrm{E}+03$ & $3.30 \mathrm{E}+03$ & $2.74 \mathrm{E}+03$ \\
\hline
\end{tabular}

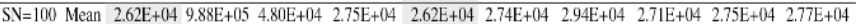
$\mathrm{D}=10 \quad \mathrm{SD} \quad 6.18 \mathrm{E}+03 \quad 8.88 \mathrm{E}+04 \quad 2.53 \mathrm{E}+04 \quad 6.19 \mathrm{E}+03 \quad 6.18 \mathrm{E}+03 \quad 6.86 \mathrm{E}+03 \quad 7.11 \mathrm{E}+03 \quad 8.25 \mathrm{E}+03 \quad 6.58 \mathrm{E}+03 \quad 7.14 \mathrm{E}+03$

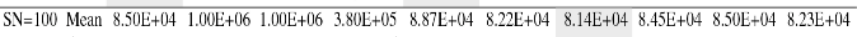
\begin{tabular}{llllllllllll}
$\mathrm{D}=30$ & $\mathrm{SD}$ & $2.12 \mathrm{E}+04$ & $0.00 \mathrm{E}+00$ & $0.00 \mathrm{E}+00$ & $2.66 \mathrm{E}+05$ & $1.93 \mathrm{E}+04$ & $1.77 \mathrm{E}+04$ & $1.82 \mathrm{E}+04$ & $1.99 \mathrm{E}+04$ & $2.12 \mathrm{E}+04$ & $1.99 \mathrm{E}+04$ \\
\hline
\end{tabular}

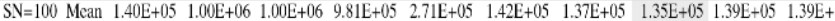

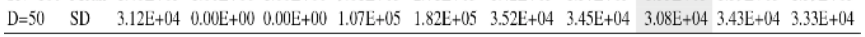

(a) Horizontal approach, $f_{1}$, (sub-)optimal solution $10^{-6}$

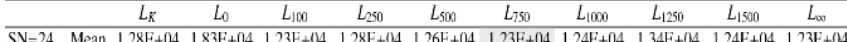
$\begin{array}{lllllllllllllll}\mathrm{D}=2 & \mathrm{SD} & 2.91 \mathrm{E}+03 & 7.27 \mathrm{E}+03 & 2.39 \mathrm{E}+03 & 3.04 \mathrm{E}+03 & 2.67 \mathrm{E}+03 & 2.22 \mathrm{E}+03 & 2.59 \mathrm{E}+03 & 2.98 \mathrm{E}+03 & 2.92 \mathrm{E}+03 & 2.27 \mathrm{E}+03\end{array}$

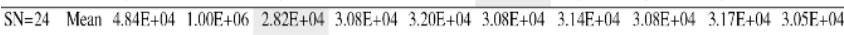

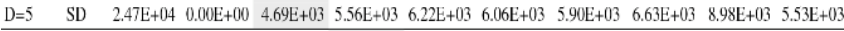

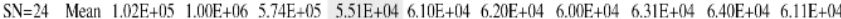
$\mathrm{D}=10 \quad$ SD $\quad 5.69 \mathrm{E}+04 \quad 0.00 \mathrm{E}+00 \quad 3.26 \mathrm{E}+05 \quad 9.39 \mathrm{E}+03 \quad 1.04 \mathrm{E}+04 \quad 1.37 \mathrm{E}+04 \quad 1.14 \mathrm{E}+04 \quad 1.40 \mathrm{E}+04 \quad 1.26 \mathrm{E}+04 \quad 1.30 \mathrm{E}+04$

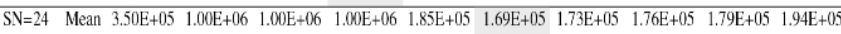
$\mathrm{D}=30 \quad \mathrm{SD} \quad 1.89 \mathrm{E}+05 \quad 0.00 \mathrm{E}+00 \quad 0.00 \mathrm{E}+00 \quad 0.00 \mathrm{E}+00 \quad 4.30 \mathrm{E}+04 \quad 2.19 \mathrm{E}+04 \quad 2.91 \mathrm{E}+04 \quad 2.72 \mathrm{E}+04 \quad 2.74 \mathrm{E}+04 \quad 4.12 \mathrm{E}+04$

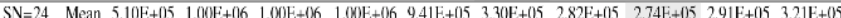
$\mathrm{D}=50 \quad$ SD $\quad 2.27 \mathrm{E}+05 \quad 0.00 \mathrm{E}+00 \quad 0.00 \mathrm{E}+00 \quad 0.00 \mathrm{E}+00 \quad 1.78 \mathrm{E}+05 \quad 8.88 \mathrm{E}+04 \quad 5.17 \mathrm{E}+04 \quad 4.00 \mathrm{E}+04 \quad 4.72 \mathrm{E}+04 \quad 6.06 \mathrm{E}+04$

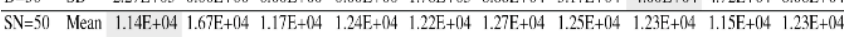
$\begin{array}{lllllllllllll}\mathrm{D}=2 & \mathrm{SD} & 1.66 \mathrm{E}+03 & 6.48 \mathrm{E}+03 & 1.77 \mathrm{E}+03 & 2.49 \mathrm{E}+03 & 2.20 \mathrm{E}+03 & 2.88 \mathrm{E}+03 & 2.53 \mathrm{E}+03 & 3.33 \mathrm{E}+03 & 2.43 \mathrm{E}+03 & 2.65 \mathrm{E}+03\end{array}$

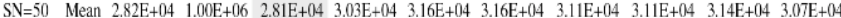
$\mathrm{D}=5 \quad \mathrm{SD} \quad 4.41 \mathrm{E}+03 \quad 0.00 \mathrm{E}+00 \quad 5.15 \mathrm{E}+03 \quad 4.50 \mathrm{E}+03 \quad 6.55 \mathrm{E}+03 \quad 8.22 \mathrm{E}+03 \quad 7.29 \mathrm{E}+03 \quad 6.42 \mathrm{E}+03 \quad 7.15 \mathrm{E}+03 \quad 5.96 \mathrm{E}+03$

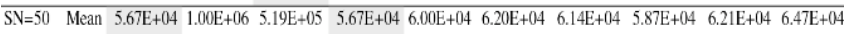
$\begin{array}{llllllllllllll}\mathrm{D}=10 & \text { SD } & 8.71 \mathrm{E}+03 & 0.00 \mathrm{E}+00 & 3.48 \mathrm{E}+05 & 8.71 \mathrm{E}+03 & 1.06 \mathrm{E}+04 & 1.43 \mathrm{E}+04 & 1.30 \mathrm{E}+04 & 1.35 \mathrm{E}+04 & 1.50 \mathrm{E}+04 & 1.58 \mathrm{E}+04\end{array}$ \begin{tabular}{llllllllllllllll}
\hline $\mathrm{SV}=50$ & Mean & $1.69 \mathrm{E}+0.0$ & $1.00 \mathrm{E}+06$ & $1.00 \mathrm{E}+06$ & $1.00 \mathrm{E}+06$ & $1.74 \mathrm{E}+05$ & $1.69 \mathrm{E}+05$ & $1.71 \mathrm{E}+05$ & $1.74 \mathrm{E}+05$ & $1.78 \mathrm{E}+05$ & $1.90 \mathrm{E}+0$.
\end{tabular} $\mathrm{D}=30 \quad \mathrm{SD} \quad 2.86 \mathrm{E}+04 \quad 0.00 \mathrm{E}+00 \quad 0.00 \mathrm{E}+00 \quad 0.00 \mathrm{E}+00 \quad 3.44 \mathrm{E}+04 \quad 2.86 \mathrm{E}+04 \quad 2.87 \mathrm{E}+04 \quad 3.26 \mathrm{E}+04 \quad 2.97 \mathrm{E}+04 \quad 4.69 \mathrm{E}+04$

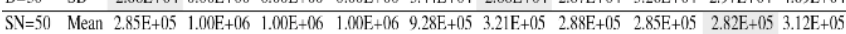
$\mathrm{D}=50 \quad$ SD $\quad 3.97 \mathrm{E}+04 \quad 0.00 \mathrm{E}+00 \quad 0.00 \mathrm{E}+00 \quad 0.00 \mathrm{E}+00 \quad 1.92 \mathrm{E}+05 \quad 8.33 \mathrm{E}+04 \quad 4.99 \mathrm{E}+04 \quad 3.97 \mathrm{E}+04 \quad 4.60 \mathrm{E}+04 \quad 7.02 \mathrm{E}+04$ $\mathrm{SV}=100 \quad$ Mean $1.19 \mathrm{E}+04 \quad 1.74 \mathrm{E}+04 \quad 1.19 \mathrm{E}+04 \quad 1.29 \mathrm{E}+04 \quad 1.30 \mathrm{E}+04 \quad 1.27 \mathrm{E}+04 \quad 1.24 \mathrm{E}+04 \quad 1.26 \mathrm{E}+04 \quad 1.24 \mathrm{E}+04 \quad 1.26 \mathrm{E}+04$ $\mathrm{D}=2 \quad \mathrm{SD} \quad 2.37 \mathrm{E}+03 \quad 6.45 \mathrm{E}+03 \quad 2.37 \mathrm{E}+03 \quad 2.68 \mathrm{E}+03 \quad 2.74 \mathrm{E}+03 \quad 4.08 \mathrm{E}+03 \quad 2.37 \mathrm{E}+03 \quad 2.75 \mathrm{E}+03 \quad 3.04 \mathrm{E}+03 \quad 2.74 \mathrm{E}+03$

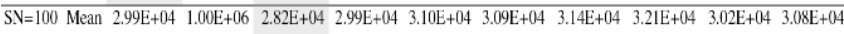
$\mathrm{D}=5 \quad \mathrm{SD} \quad 5.50 \mathrm{E}+03 \quad 0.00 \mathrm{E}+00 \quad 3.84 \mathrm{E}+03 \quad 5.50 \mathrm{E}+03 \quad 6.88 \mathrm{E}+03 \quad 5.87 \mathrm{E}+03 \quad 6.25 \mathrm{E}+03 \quad 7.16 \mathrm{E}+03 \quad 6.18 \mathrm{E}+03 \quad 5.56 \mathrm{E}+03$ $\mathrm{SV}=100$ Mean $\quad 5.85 \mathrm{E}+04 \quad 1.00 \mathrm{~F}+06 \quad 5.73 \mathrm{E}+05 \quad 5.62 \mathrm{~F}+04 \quad 5.85 \mathrm{E}+04 \quad 6.16 \mathrm{~F}+04 \quad 6.49 \mathrm{E}+04 \quad 6.17 \mathrm{E}+04 \quad 6.19 \mathrm{~F}+04 \quad 6.04 \mathrm{E}+0.4$ $\mathrm{D}=10 \quad \mathrm{SD} \quad 1.05 \mathrm{E}+04 \quad 0.00 \mathrm{E}+00 \quad 3.40 \mathrm{E}+05 \quad 8.70 \mathrm{E}+03 \quad 1.05 \mathrm{E}+04 \quad 1.33 \mathrm{E}+04 \quad 1.39 \mathrm{E}+04 \quad 1.07 \mathrm{E}+04 \quad 1.19 \mathrm{E}+04 \quad 1.47 \mathrm{E}+04$

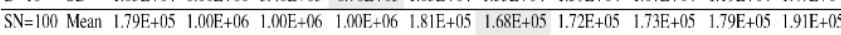
$\mathrm{D}=30 \quad$ SD $\quad 3.56 \mathrm{E}+04 \quad 0.00 \mathrm{E}+00 \quad 0.00 \mathrm{E}+00 \quad 0.00 \mathrm{E}+00 \quad 4.51 \mathrm{E}+04 \quad 2.62 \mathrm{E}+04 \quad 2.61 \mathrm{E}+04 \quad 3.49 \mathrm{E}+04 \quad 3.56 \mathrm{E}+04 \quad 5.18 \mathrm{E}+04$ $\begin{array}{lllllllllllllllll}\mathrm{SN}=100 & \text { Mean } & 3.02 \mathrm{E}+05 & 1.00 \mathrm{E}+06 & 1.00 \mathrm{E}+06 & 1.00 \mathrm{E}+06 & 9.38 \mathrm{E}+05 & 3.08 \mathrm{E}+05 & 2.84 \mathrm{E}+05 & 2.88 \mathrm{E}+05 & 2.86 \mathrm{E}+05 & 3.17 \mathrm{E}+0.5\end{array}$

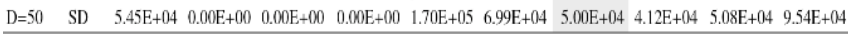


other fixed 'limit' values performed better; in most cases (8 out of 15), the better performing value being $L_{250}$. A meticulous reader may notice that those problems with higher dimensions always required more fitness evaluations in order to reach a sub-optimal solution (Tables 2(a) and 2(b)), which was an expected property. Less expectedly, the population size did not have a big influence on this property. For example, to reach $10^{-6}$, the following average numbers of fitness evaluations were needed at $L_{1000}: 5.65 \mathrm{E}+03(S N=$ $100, D=2), 5.60 \mathrm{E}+03(S N=50, D=2)$, and $5.31 \mathrm{E}+03$ $(S N=24, D=2)$, whilst 8.14E+04 $(S N=100, D=30)$, 8.17 $\mathrm{E}+04(S N=50, D=30)$, and 8.22E $+04(S N=24, D=$ 30 ). In order to reach $10^{-12}$, twice as many fitness evaluations were roughly needed compared to $10^{-6}$. Again, an increase in the number of fitness evaluations was expected, although the magnitude of the increase was hard to predict. From these tables, as well as based on the results for $f_{2}$ to $f_{5}$ (not shown in this paper), we noticed that setting the 'limit' was a difficult task. It can be observed that setting the control parameter 'limit' using the formula from [26] obtained good results only for the vertical approach with 250,000 fitness evaluations. If only one experiment were applied, the wrong conclusions could be drawn. In other cases, a clear winner was hard to discover (if it existed at all). However, we could not define a rule for setting 'limit' based on these results as the statistical significance had not yet been examined.

Figure 1 shows the sensitivity analyses for the (a) vertical approach with a maximum number of 100,000 fitness evaluations; (b) vertical approach with a maximum number of 250,000 fitness evaluations; (c) horizontal approach with (sub-)optimal solution $10^{-6}$; (d)

Figure 1

Sensitivity analyses of $f_{1}$ using horizontal and vertical approaches

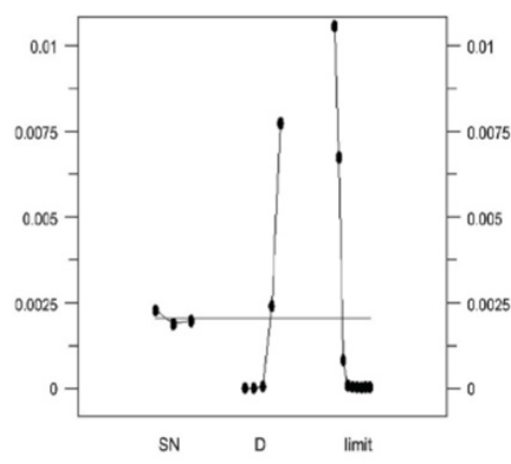

(a) Vertical approach, MaxFEs $=100,000$

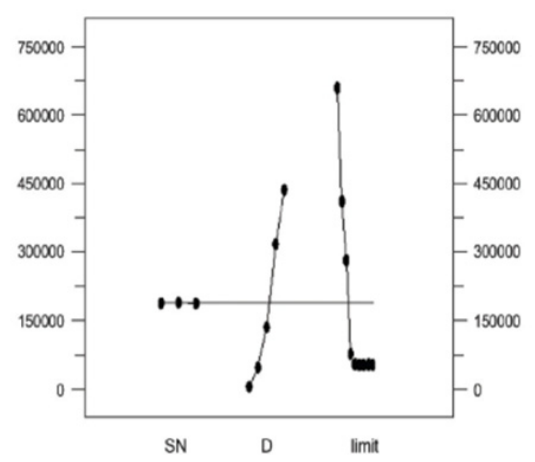

(c) Horizontal approach, $10^{-6}$

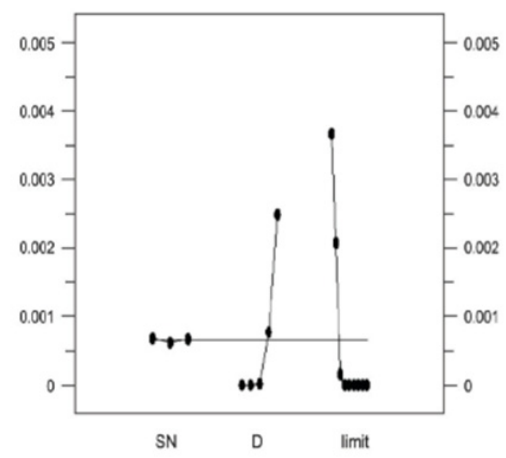

(b) Vertical approach, MaxFEs $=250,000$

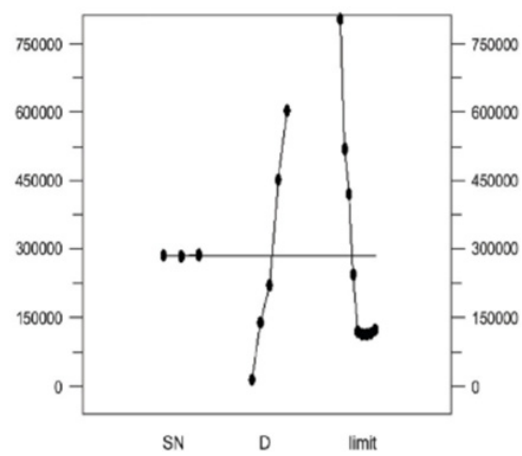

(d) Horizontal approach, $10^{-12}$ 
horizontal approach with (sub-)optimal solution $10^{-12}$. The X-axis represents the parameter settings of $S N(3$ settings: 100, 50, 24 from left to right), $D$ (5 settings: 2 , $5,10,30,50$ from left to right), and 'limit' (9 settings: $0,100,250,500,750,1000,1250,1500, \infty$ from left to right). The Y-axis represents the sensitivities of three parameters in terms of the average of better solutions found and the average number of fitness evaluations needed to reach (sub-)optimal solution amongst 100 runs for vertical and horizontal approaches, respectively.

As can be observed, $S N$ had minimal effect. Changing $S N$ amongst 24, 50, and 100 did not make too much difference. Conversely, 'limit' and $D$ played important roles when determining the performance of the $A B C$ algorithm. All the figures indicated that 'limit' was more sensitive than $D$ because, in terms of the Y-axis, the range of 'limit' was longer than $D$. Conversely, $D$ is not the $\mathrm{ABC}$ control parameter but the property of the problem. Hence, amongst ABC control parameters the size of the population $(S N)$ was much more robust than control parameter 'limit', indicating that much more emphasis should be given to properly setting it.

All four graphs in Figure 1 show remarkable similarities, and although they show that $A B C$ is very sensitive to 'limit', an important question is: "Are differences in setting 'limit' also statistically significant?" Hence, we performed NHST and CRS4EAs analyses on the obtained results. Furthermore, all four graphs in Figure 1 clearly indicate that there exists no linear relationship between 'limit', population size $S N$, and dimension $D$, as suggested by formula [26].

\subsection{Null Hypothesis Significance Testing}

Karaboga's suggestion of 'limit' value $L_{k}=n_{e}{ }^{*} D=$ $(S N / 2) * D[26]$ was compared to the set of fixed 'limit' values 'limit' $=\{0,100,250,500,750,1000,1250,1500$,

\section{Table 3}

Description of all four parts of the experiment

\begin{tabular}{llll}
\hline Section & Approach & Termination condition & Measurement \\
\hline Experiment I & vertical & number of fitness evaluations is 100,000 & quality of solution \\
Experiment 2 & vertical & number of fitness evaluations is 250,000 & quality of solution \\
Experiment 3 & horizontal & (sub-)optimal solution $10^{-6}$ & number of fitness evaluations \\
& & and maximum of fitness evaluations is 1,000,000 & \\
Experiment 4 & horizontal & (sub-)optimal solution $10^{-12}$ & number of fitness evaluations \\
& & and maximum of fitness evaluations is $1,000,000$ \\
\hline
\end{tabular}

$\infty\}$ for $S N=\{24,50,100\}$ and $D=\{2,5,10,30,50\}$. The whole experiment was divided into four sections (see Table 3). In the first two sections, we measured the quality of a solution reached by a pre-defined number of fitness evaluations $(100,000$ and 250,000), which is also known as the vertical or 'the fixed-cost' approach. In the other two sections, we measured the number of fitness evaluations needed to find a (sub-) optimal solution $\left(10^{-6}\right.$ and $\left.10^{-12}\right)$, which is also known as the horizontal or 'the fixed-target' approach. The horizontal approach would have stopped the algorithm if a (sub-)optimal solution could not be found over 1,000,000 fitness evaluations. The number of independent runs was in all cases $n=100$. By using the vertical approach only the quality of the final solution was taken into consideration but not the convergence. Fast convergence is also a desirable property of meta-heuristic algorithms, which can be captured using the horizontal approach. Convergence can also be analysed by using the vertical approach and additional Page's trend statistics, as shown in [10].

The obtained results (readers can find the raw data in [47]) were analysed using Null Hypothesis Significance Testing [41] for multiple comparisons. The non-parametric Wilcoxon's test [51] was used because the distribution of the data was unknown. In Wilcoxon's test, the results $L_{k}$ obtained over $n=100$ runs for particular settings $S N, D$ and problem $f_{i}$ were pairwisely compared to the results of another fixed 'limit' value obtained over $n=100$ runs for the same settings $S N, D$ and problem $f_{i}$. The differences between the corresponding outcomes were ranked and the $p$ value was calculated regarding to the sum of positive ranks (whenever $L_{k}$ was better) and the sum of negative ranks (whenever $L_{k}$ was worse). As several multiple Wilcoxon's tests were conducted on the same data and we wished to control the Type-IError, the post-hoc procedure known as the Holm test [19] was applied to each such comparison. In Holm's procedure, $p$ values (there is $k=9$ of them) obtained using Wilcoxon's test were ordered from the most significant (smallest $p$ value, i.e., $p_{1}$ ) to the least significant (largest $p$ value, i.e., $p_{k}$ ). $p_{1}$ was then compared to $\alpha /(k-1)$, and if it was smaller, the hypothesis (which states that $L_{k}$ and 'limit' setting linked to $p_{1}$ are equal) was rejected. $p_{2}$ was compared to $\alpha /(k-2), p_{3}$ to $\alpha /(k$ -3) and so on, until the value $j$ for which $p_{j}$ was not smaller than $\alpha /(k-j)$ was found. When such a $j$ was 
found, the procedure stopped and all the remaining hypotheses were retained. All the results from the presented experiments were analysed under a significance level of $\alpha=0.05$. The results of these analyses are presented in Tables 5-21. In each table, Karaboga's 'limit' value $L_{k}$ is compared to other fixed values of 'limit' $\left(L_{0}, L_{100}, L_{250}, L_{500}, L_{750}, L_{1000}, L_{1250}, L_{1500}, L_{\infty}\right) . L_{k}$ was either better $(>)$, equal $(=)$, or worse $(<)$ than any fixed value of 'limit'. The decision whether $L_{k}$ was better or worse depended on the sums of the positive and negative ranks from the Wilcoxon's test. Whenever the difference between the two values was significant under the Holm test, there is a star symbol (*) behind the 'limit' value. Whenever Karaboga's 'limit' value $L_{k}$ was worse than at least one other fixed 'limit' value, the cell in the table is highlighted in light grey colour. Since $L_{k}$ was different for different settings of $S N$ and $D$, its values are displayed in Table 4 .

\section{Table 4}

Values of 'limit' $L_{k}=(S N / 2)^{*} D$

\begin{tabular}{lllllllll}
\hline & $\mathrm{D}=2$ & $\mathbf{D}=5$ & $\mathbf{D}=\mathbf{1 0}$ & $\mathbf{D}=\mathbf{3 0}$ & $\mathrm{D}=\mathbf{5 0}$ & $\mathrm{D}=100$ & $\mathrm{D}=200$ & $\mathrm{D}=300$ \\
\hline $\mathrm{SN}=24$ & $L_{K}=24$ & $L_{K}=60$ & $L_{K}=120$ & $L_{K}=360$ & $L_{K}=600$ & $L_{K}=1200$ & $L_{K}=2400$ & $L_{K}=3600$ \\
$\mathrm{SN}=50$ & $L_{K}=50$ & $L_{K}=125$ & $L_{K}=250$ & $L_{K}=750$ & $L_{K}=1250$ & $L_{K}=2500$ & $L_{K}=5000$ & $L_{K}=7500$ \\
$\mathrm{SN}=100$ & $L_{K}=100$ & $L_{K}=250$ & $L_{K}=500$ & $L_{K}=1500$ & $L_{K}=2500$ & $L_{K}=5000$ & $L_{K}=10000$ & $L_{K}=15000$
\end{tabular}

\subsubsection{Experiment 1: Vertical Approach with MaxFEs $=100,00$}

Tables 5-9 show the differences found between $L_{k}$ and the other 9 fixed 'limit' values on all 5 optimisa- tion problems. While $L_{k}$ was in most cases better than some fixed 'limit' values, there were some values for which $L_{k}$ was worse, sometimes even significantly. In particular, for $f_{1}: S N=24$ and $D=5$ where $L_{k}$ was significantly worse than $L_{100}, L_{250}, L_{500}, L_{750}, L_{1000}, L_{1250}$, $L_{1500}, L_{\infty} ; S N=24$ and $\mathrm{D}=10$ where $L_{k}$ was significantly worse than $L_{250}, L_{500}, L_{750}, L_{1000}, L_{1250}, L_{1500} ; S N=100$ and $D=10$ where $L_{k}$ was significantly worse than $L_{250}$; $S N=24$ and $D=30$ where $L_{k}$ was significantly worse than $L_{500}, L_{750}, L_{1000}, L_{1250}, L_{1500}$. For $f_{5}: S N=24$ and $D=$ 5 where $L_{k}$ was significantly worse than $L_{100}, L_{250}, L_{500}$, $L_{750}, L_{1000}, L_{1250}, L_{1500}, L_{\infty} ; S N=50$ and $D=5$ where $L_{k}$ was significantly worse than $L_{500}, L_{750}, L_{1000}, L_{1250}, L_{1500} ; S N$ $=100$ and $D=5$ where $L_{k}$ was significantly worse than $L_{1500}$; $\mathrm{SN}=24$ and $D=10$ where $L_{k}$ was significantly worse than $L_{250}, L_{500}, L_{750}, L_{1000}, L_{1250}, L_{1500}, L_{\infty}$. Hence, $L_{k}$ had significantly better alternatives for problems $f_{1}$ and $f_{5}$, whilst for $f_{2}, f_{3}$, and $f_{4}$ the found differences were not significant. The differences between $L_{k}$ and some other fixed 'limit' values for $f_{1}$ were significant when the population size $S N$ equaled 24, and dimension $D$ equaled 5,10 , or 30 . So for this problem and small population size, $L_{k}$ would not be a better choice. For $f_{5}, L_{k}$ had significantly better alternatives whenever dimension $D$ equaled 5 , and for dimension $D=10$ and small population size $S N=24$. However, for all five problems, $L_{k}$ had better alternatives (however, these alternatives were not significantly better) when dimension $D$ was bigger (10, 30, or 50$)$ and population size $S N$ had different values.

Table 5

$f_{1}$, vertical approach, MaxFEs $=100,000$, NHST

\begin{tabular}{|c|c|c|c|c|c|c|}
\hline & & $\mathrm{SN}=24$ & & $\mathrm{SN}=50$ & & $\mathrm{SN}=100$ \\
\hline $\mathrm{D}=2$ & $\begin{array}{l}L_{K}> \\
L_{K}= \\
L_{K}<\end{array}$ & $\begin{array}{l}L_{0}^{*}, L_{500}, L_{1000}, L_{1250}, L_{1500}, L_{\infty} \\
L_{100}, L_{250}, L_{750}\end{array}$ & $\begin{array}{l}L_{K}> \\
L_{K}= \\
L_{K}<\end{array}$ & $\begin{array}{l}L_{0} *, L_{1000}, L_{1250}, L_{\infty} \\
L_{100}, L_{250}, L_{500}, L_{750}, L_{1500}\end{array}$ & $\begin{array}{l}L_{K}> \\
L_{K}= \\
L_{K}<\end{array}$ & $\begin{array}{l}L_{0} *, L_{1000}, L_{1250}, L_{1500}, L_{\infty} \\
L_{100}, L_{250}, L_{500}, L_{750}\end{array}$ \\
\hline \multirow[t]{2}{*}{$\mathrm{D}=5$} & $\begin{array}{l}L_{K}> \\
L_{K}=\end{array}$ & $L_{0} *$ & $\begin{array}{l}L_{K}> \\
L_{K}=\end{array}$ & $\begin{array}{l}L_{0} *, L_{500}, L_{750}, L_{1000}, L_{1250}, L_{1500}, L_{\infty} \\
L_{100}, L_{250}\end{array}$ & $\begin{array}{l}L_{K}> \\
L_{K}=\end{array}$ & $L_{0} *, L_{750}, L_{1000}, L_{1250}, L_{1500}$ \\
\hline & $L_{K}<$ & $\begin{array}{l}L_{100^{*},} L_{250^{*},} L_{500 *}, \quad L_{750 *}, L_{1000^{*}}, \\
L_{1250^{*}, L_{1500^{*},} L_{\infty} *}\end{array}$ & $L_{K}<$ & & $L_{K}<$ & $L_{100}, L_{250}, L_{500}, L_{\infty}$ \\
\hline \multirow{3}{*}{$\mathrm{D}=10$} & $L_{K}>$ & $L_{0} *, L_{100} *$ & $L_{K}>$ & $\begin{array}{l}L_{0} *, L_{100} *, L_{500}, L_{750} *, L_{1000} *, L_{1250} * \\
L_{1500 *, L_{\infty} *}\end{array}$ & $L_{K}>$ & $\begin{array}{l}L_{0} *, L_{100} *, L_{750} *, L_{1000}, L_{1500}, L_{1250}, \\
L_{\infty}\end{array}$ \\
\hline & $L_{K}=$ & & $L_{K}=$ & $L_{250}$ & $L_{K}=$ & $L_{500}$ \\
\hline & $L_{K}<$ & $\begin{array}{l}L_{250 *}, L_{500 *}, L_{750} *, L_{1000} *, L_{1250 *}, \\
L_{1500^{*},} L_{\infty} *\end{array}$ & $L_{K}<$ & & $L_{K}<$ & $L_{250 *}$ \\
\hline \multirow[t]{2}{*}{$\mathrm{D}=\mathbf{3 0}$} & $\begin{array}{l}L_{K}> \\
L_{K}=\end{array}$ & $L_{0} *, L_{100} *, L_{250} *$ & $\begin{array}{l}L_{K}> \\
L_{K}=\end{array}$ & $\begin{array}{l}L_{0} *, L_{100} *, L_{250} *, L_{500}, L_{1000} \\
L_{750}\end{array}$ & $\begin{array}{l}L_{K}> \\
L_{K}=\end{array}$ & $\begin{array}{l}L_{0} *, L_{100} *, L_{250} *, L_{500}, L_{1000}, L_{\infty} \\
L_{1500}\end{array}$ \\
\hline & $L_{K}<$ & $\begin{array}{l}L_{500^{*},} L_{750} *, L_{1000} *, L_{1250} *, L_{1500} * \\
L_{\infty} *\end{array}$ & $L_{K}<$ & $L_{1250}, L_{1500}, L_{\infty}$ & $L_{K}<$ & $L_{750}, L_{1250}$ \\
\hline \multirow[t]{2}{*}{$\mathrm{D}=50$} & $\begin{array}{l}L_{K}> \\
L_{K}=\end{array}$ & $L_{0} *, L_{100} *, L_{250} *, L_{500} *$ & $\begin{array}{l}L_{K}> \\
L_{K}=\end{array}$ & $\begin{array}{l}L_{0} *, L_{100} *, L_{250} *, L_{500} *, L_{1000} \\
L_{1250}\end{array}$ & $\begin{array}{l}L_{K}> \\
L_{K}=\end{array}$ & $L_{0} *, L_{100} *, L_{250} *, L_{500} *, L_{750}$ \\
\hline & $L_{K}<$ & $L_{750}, L_{1000}, L_{1250}, L_{1500}, L_{\infty}$ & $L_{K}<$ & $L_{750}, L_{1500}, L_{\infty}$ & $L_{K}<$ & $L_{1000}, L_{1250}, L_{1500}, L_{\infty}$ \\
\hline
\end{tabular}


Table 6

$f_{2}$, vertical approach, MaxFEs $=100,000$, NHST

\begin{tabular}{|c|c|c|c|c|c|c|}
\hline & & $\mathrm{SN}=24$ & & $\mathrm{SN}=50$ & & $\mathrm{SN}=100$ \\
\hline $\mathrm{D}=2$ & $\begin{array}{l}L_{K}> \\
L_{K}= \\
L_{K}<\end{array}$ & $\begin{array}{l}L_{0} *, L_{100}, L_{250} *, L_{500} *, L_{750} *, L_{1000} * \\
L_{1250} *, L_{1500} *, L_{\infty} *\end{array}$ & $\begin{array}{l}L_{K}> \\
L_{K}= \\
L_{K}<\end{array}$ & $\begin{array}{l}L_{0} *, L_{100}, L_{250} *, L_{500} *, L_{750} *, L_{1000} * \\
L_{1250} *, L_{1500} *, L_{\infty} *\end{array}$ & $\begin{array}{l}L_{K}> \\
L_{K}= \\
L_{K}<\end{array}$ & $\begin{array}{l}L_{0} *, L_{250} *, L_{500} *, L_{750} *, L_{1000} *, L_{1250} * \\
L_{1500} *, L_{\infty} * \\
L_{100}\end{array}$ \\
\hline $\mathrm{D}=\mathbf{5}$ & $\begin{array}{l}L_{K}> \\
L_{K}= \\
L_{K}<\end{array}$ & $\begin{array}{l}L_{0} *, L_{100}, L_{500}, L_{750} *, L_{1000}, L_{1250} * \\
L_{1500} *, L_{\infty} * \\
L_{250}\end{array}$ & $\begin{array}{l}L_{K}> \\
L_{K}= \\
L_{K}<\end{array}$ & $\begin{array}{l}L_{0} *, L_{100}, L_{250}, L_{500} *, L_{750} *, L_{1000} * \\
L_{1250} *, L_{1500} *, L_{\infty} *\end{array}$ & $\begin{array}{l}L_{K}> \\
L_{K}= \\
L_{K}<\end{array}$ & $\begin{array}{l}L_{0} *, L_{100} *, L_{500} *, L_{750} *, L_{1000} *, L_{1250} * \\
L_{1500} *, L_{\infty} * \\
L_{250}\end{array}$ \\
\hline$D=10$ & $\begin{array}{l}L_{K}= \\
L_{K}<\end{array}$ & $L_{0} *, L_{250}, L_{1000}, L_{1250}, L_{1500}$ & $\begin{array}{l}L_{K}= \\
L_{K}<\end{array}$ & $\begin{array}{l}L_{0} *, L_{100}, L_{500}, L_{750}, L_{1000}, L_{1250}, \\
L_{1500}, L_{\infty} \\
L_{250}\end{array}$ & $\begin{array}{l}L_{K}= \\
L_{K}<\end{array}$ & $L_{0} *, L_{250}, L_{750}, L_{1000}, L_{1250}, L_{\infty}$ \\
\hline $\mathrm{D}=\mathbf{3 0}$ & $\begin{array}{l}L_{K}> \\
L_{K}= \\
L_{K}<\end{array}$ & $\begin{array}{l}L_{0} *, L_{100}, L_{500}, L_{750}, L_{1000}, L_{1250}, L_{\infty} \\
L_{250}, L_{1500}\end{array}$ & $\begin{array}{l}L_{K}> \\
L_{K}= \\
L_{K}<\end{array}$ & $\begin{array}{l}L_{0} *, L_{250}, L_{500}, L_{\infty} \\
L_{750} \\
L_{100}, L_{1000}, L_{1250}, L_{1500}\end{array}$ & $\begin{array}{l}L_{K}> \\
L_{K}= \\
L_{K}<\end{array}$ & $\begin{array}{l}L_{0} *, L_{250}, L_{750}, L_{1250}, L_{\infty} \\
L_{1500} \\
L_{500}, L_{1000}\end{array}$ \\
\hline $\mathrm{D}=\mathbf{5 0}$ & $\begin{array}{l}L_{K}> \\
L_{K}= \\
L_{K}<\end{array}$ & $\begin{array}{l}L_{0} *, L_{1000} \\
L_{100}, L_{250}, L_{500}, L_{750}, L_{1250}, L_{1500}, L_{\infty}\end{array}$ & $\begin{array}{l}L_{K}> \\
L_{K}= \\
L_{K}<\end{array}$ & $\begin{array}{l}L_{0} *, L_{100}, L_{250}, L_{750}, L_{1000}, L_{1500}, L_{\infty} \\
L_{1250} \\
L_{500}\end{array}$ & $\begin{array}{l}L_{K}> \\
L_{K}= \\
L_{K}<\end{array}$ & $\begin{array}{l}L_{0} *, L_{100}, L_{250}, L_{1000}, L_{1500} \\
L_{500}, L_{750}, L_{1250}, L_{\infty}\end{array}$ \\
\hline
\end{tabular}

\section{Table 7}

$f_{3}$, vertical approach, MaxFEs $=100,000$, NHST

\begin{tabular}{|c|c|c|c|c|c|c|}
\hline & & $\mathrm{SN}=\mathbf{2 4}$ & & $\mathrm{SN}=50$ & & $\mathrm{SN}=100$ \\
\hline \multirow[b]{2}{*}{$\mathrm{D}=2$} & $L_{K}>$ & $L_{0} *$ & $L_{K}>$ & $L_{0} *$ & $L_{K}>$ & $L_{0} *$ \\
\hline & $L_{K}=$ & $\begin{array}{l}L_{100}, L_{250}, L_{500}, L_{750}, L_{1000}, L_{1250}, \\
L_{1500}, L_{\infty}\end{array}$ & $L_{K}=$ & $\begin{array}{l}L_{100}, L_{250}, L_{500}, L_{750}, L_{1000}, L_{1250}, \\
L_{1500}, L_{\infty}\end{array}$ & $L_{K}=$ & $\begin{array}{l}L_{100}, L_{250}, L_{500}, L_{750}, L_{1000}, L_{1250}, \\
L_{1500}, L_{\infty}\end{array}$ \\
\hline \multirow{3}{*}{$\mathrm{D}=5$} & $L_{K}>$ & $L_{0} *, L_{1250}, L_{1500}, L_{\infty}$ & $L_{K}>$ & $L_{0} *, L_{750}, L_{1250}, L_{1500}, L_{\infty}$ & $L_{K}>$ & $L_{0} *, L_{750}, L_{1250}, L_{1500}, L_{\infty}$ \\
\hline & $\begin{array}{l}L_{K}= \\
L_{K}<\end{array}$ & $L_{100}, L_{250}, L_{500}, L_{750}, L_{1000}$ & $\begin{array}{l}L_{K}= \\
L_{K}<\end{array}$ & $L_{100}, L_{250}, L_{500}, L_{1000}$ & $\begin{array}{l}L_{K}= \\
L_{K}<\end{array}$ & $L_{100}, L_{250}, L_{500}, L_{1000}$ \\
\hline & $L_{K}>$ & $\begin{array}{l}L_{0} *, L_{100}, L_{250}, L_{500}, L_{750}, L_{1000}, L_{1250}, \\
L_{1500}, L_{\infty}\end{array}$ & $L_{K}>$ & $L_{0} *, L_{500}$ & $L_{K}>$ & $L_{0} *, L_{750}$ \\
\hline \multirow[t]{2}{*}{$\mathrm{D}=\mathbf{1 0}$} & $L_{K}=$ & & $L_{K}=$ & $L_{250}, L_{750}, L_{1000}, L_{1500}$ & $L_{K}=$ & $L_{500}$ \\
\hline & $L_{K}<$ & & $L_{K}<$ & $L_{100}, L_{1250}, L_{\infty}$ & $L_{K}<$ & $L_{100}, L_{250}, L_{1000}, L_{1250}, L_{1500}, L_{\infty}$ \\
\hline \multirow[t]{2}{*}{$\mathrm{D}=\mathbf{3 0}$} & $\begin{array}{l}L_{K}> \\
L_{K}=\end{array}$ & $L_{0} *, L_{100}, L_{1500}$ & $\begin{array}{l}L_{K}> \\
L_{K}=\end{array}$ & $\begin{array}{l}L_{0} *, L_{250}, L_{500}, L_{1000}, L_{1250}, L_{\infty} \\
L_{750}\end{array}$ & $\begin{array}{l}L_{K}> \\
L_{K}=\end{array}$ & $\begin{array}{l}L_{0} *, L_{100}, L_{250}, L_{750} *, L_{1000}, L_{1250}, L_{\infty} \\
L_{1500}\end{array}$ \\
\hline & $L_{K}<$ & $L_{250}, L_{500}, L_{750}, L_{1000}, L_{1250}, L_{\infty}$ & $L_{K}<$ & $L_{100}, L_{1500}$ & $L_{K}<$ & $L_{500}$ \\
\hline \multirow[b]{2}{*}{$\mathrm{D}=\mathbf{5 0}$} & $L_{K}>$ & $L_{0} *, L_{100}, L_{750}, L_{1250}, L_{1500}$ & $L_{K}>$ & $\begin{array}{l}L_{0} *, L_{100}, L_{250}, L_{500}, L_{750}, L_{1000}, L_{1500}, \\
L_{\infty}\end{array}$ & $L_{K}>$ & $\begin{array}{l}L_{0} *, L_{100}, L_{250}, L_{500}, L_{1000}, L_{1250} \\
L_{1500}, L_{\infty}\end{array}$ \\
\hline & $L_{K}=$ & & $L_{K}=$ & $L_{1250}$ & $L_{K}=$ & \\
\hline
\end{tabular}

\section{Table 8}

$f_{4}$, vertical approach, MaxFEs $=100,000$, NHST

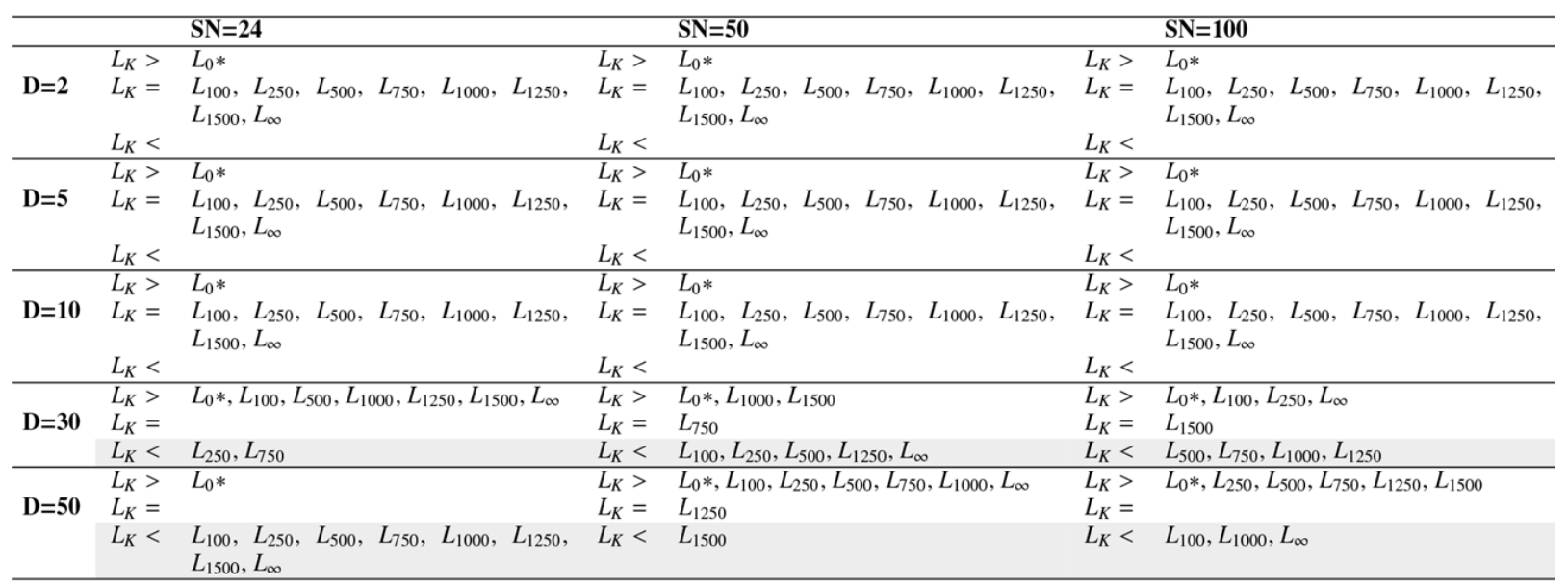




\section{Table 9}

$f_{5}$, vertical approach, MaxFEs $=100,000$, NHST

\begin{tabular}{|c|c|c|c|c|c|c|}
\hline & & $\mathrm{SN}=24$ & & $\mathrm{SN}=50$ & & $\mathrm{SN}=100$ \\
\hline$D=2$ & $\begin{array}{l}L_{K}> \\
L_{K}= \\
L_{K}<\end{array}$ & $\begin{array}{l}L_{0} *, L_{\infty} \\
L_{100}, L_{250}, L_{500}, L_{750}, L_{1000}, L_{1250}, \\
L_{1500}\end{array}$ & $\begin{array}{l}L_{K}> \\
L_{K}= \\
L_{K}<\end{array}$ & $\begin{array}{l}L_{0} *, L_{\infty} \\
L_{100}, L_{250}, L_{500}, L_{750}, L_{1000}, L_{1250}, \\
L_{1500}\end{array}$ & $\begin{array}{l}L_{K}> \\
L_{K}= \\
L_{K}<\end{array}$ & $\begin{array}{l}L_{0} *, L_{\infty} \\
L_{100}, L_{250}, L_{500}, L_{750}, L_{1000}, L_{1250}, \\
L_{1500}\end{array}$ \\
\hline$D=5$ & $\begin{array}{l}L_{K}> \\
L_{K}= \\
L_{K}<\end{array}$ & $\begin{array}{l}L_{0} *, L_{\infty} * \\
L_{100}, L_{250}, L_{500}, L_{750}, L_{1000}, L_{1250}, \\
L_{1500}\end{array}$ & $\begin{array}{l}L_{K}> \\
L_{K}= \\
L_{K}<\end{array}$ & $\begin{array}{l}L_{0} *, L_{\infty} \\
L_{100}, L_{250}, L_{500}, L_{750}, L_{1000}, L_{1250}, \\
L_{1500}\end{array}$ & $\begin{array}{l}L_{K}> \\
L_{K}= \\
L_{K}<\end{array}$ & $\begin{array}{l}L_{0} *, L_{1000}, L_{1250}, L_{\infty} \\
L_{100}, L_{250}, L_{500}, L_{750}, L_{1500}\end{array}$ \\
\hline$D=10$ & $\begin{array}{l}L_{K}> \\
L_{K}= \\
L_{K}<\end{array}$ & $\begin{array}{l}L_{0} *, L_{100} *, L_{1250}, L_{\infty} \\
L_{250}, L_{500}, L_{750}, L_{1000}, L_{1500}\end{array}$ & $\begin{array}{l}L_{K}> \\
L_{K}= \\
L_{K}<\end{array}$ & $\begin{array}{l}L_{0} *, L_{100} *, L_{1250}, L_{\infty} \\
L_{250}, L_{500}, L_{750}, L_{1000}, L_{1500}\end{array}$ & $\begin{array}{l}L_{K}> \\
L_{K}= \\
L_{K}<\end{array}$ & $\begin{array}{l}L_{0} *, L_{100} *, L_{1000}, L_{1250}, L_{1500}, L_{\infty} \\
L_{250}, L_{500}, L_{750}\end{array}$ \\
\hline$D=30$ & $\begin{array}{l}L_{K}> \\
L_{K}= \\
L_{K}<\end{array}$ & $\begin{array}{l}L_{0} *, L_{100} *, L_{250} *, L_{500} *, L_{750} *, L_{1000} * \\
L_{1250} *, L_{1500} *, L_{\infty} *\end{array}$ & $\begin{array}{l}L_{K}> \\
L_{K}= \\
L_{K}<\end{array}$ & $\begin{array}{l}L_{0} *, L_{100} *, L_{250} *, L_{500} *, L_{1000} *, L_{1250} * \\
L_{1500} *, L_{\infty} * \\
L_{750}\end{array}$ & $\begin{array}{l}L_{K}> \\
L_{K}= \\
L_{K}<\end{array}$ & $\begin{array}{l}L_{0} *, L_{100} *, L_{250} *, L_{500} *, L_{750} *, L_{1000} * \\
L_{1250} *, L_{\infty} * \\
L_{1500}\end{array}$ \\
\hline$D=50$ & $\begin{array}{l}L_{K}> \\
L_{K}= \\
L_{K}<\end{array}$ & $\begin{array}{l}L_{0} *, L_{100} *, L_{250} *, L_{500} *, L_{750} *, L_{1000} * \\
L_{1250} * L_{1500} * L_{\infty} *\end{array}$ & $\begin{array}{l}L_{K}> \\
L_{K}= \\
L_{K}<\end{array}$ & $\begin{array}{l}L_{0} *, L_{100} *, L_{250} *, L_{500} *, L_{750} *, L_{1000} * \\
L_{1500} *, L_{\infty} * \\
L_{1250}\end{array}$ & $\begin{array}{l}L_{K}> \\
L_{K}= \\
L_{K}<\end{array}$ & $\begin{array}{l}L_{0} *, L_{100} *, L_{250} *, L_{500} *, L_{750} *, L_{1000} * \\
L_{1250} *, L_{1500} *, L_{\infty} *\end{array}$ \\
\hline
\end{tabular}

\section{Table 10}

$f_{1}$, vertical approach, MaxFEs $=250,000$, NHST

\begin{tabular}{|c|c|c|c|c|c|c|}
\hline & & $\mathrm{SN}=24$ & & $\mathrm{SN}=50$ & & $\mathrm{SN}=100$ \\
\hline$D=2$ & $\begin{array}{l}L_{K}> \\
L_{K}= \\
L_{K}<\end{array}$ & $\begin{array}{l}L_{0} *, L_{100} *, L_{250} *, L_{500} *, L_{750} *, L_{1000} * \\
L_{1250}, L_{1500} *, L_{\infty} *\end{array}$ & $\begin{array}{l}L_{K}> \\
L_{K}= \\
L_{K}<\end{array}$ & $\begin{array}{l}L_{0} *, L_{100} *, L_{250} *, L_{500} *, L_{750} *, L_{1000} * \\
L_{1250}, L_{1500} * L_{\infty} *\end{array}$ & $\begin{array}{l}L_{K}> \\
L_{K}= \\
L_{K}<\end{array}$ & $\begin{array}{l}L_{0} *, L_{250} *, L_{500} *, L_{750} *, L_{1000} *, L_{1250} * \\
L_{1500} *, L_{\infty} * \\
L_{100}\end{array}$ \\
\hline$D=5$ & $\begin{array}{l}L_{K}> \\
L_{K}= \\
L_{K}<\end{array}$ & $\begin{array}{l}L_{0} *, L_{100} *, L_{250} *, L_{500} *, L_{750} *, L_{1000} * \\
L_{1250} *, L_{1500} *, L_{\infty} *\end{array}$ & $\begin{array}{l}L_{K}> \\
L_{K}= \\
L_{K}<\end{array}$ & $\begin{array}{l}L_{0} *, L_{100} *, L_{250} *, L_{500} *, L_{750} *, L_{1000} * \\
L_{1250} *, L_{1500} *, L_{\infty} *\end{array}$ & $\begin{array}{l}L_{K}> \\
L_{K}= \\
L_{K}<\end{array}$ & $\begin{array}{l}L_{0} *, L_{100} *, L_{500} *, L_{750} *, L_{1000} *, L_{1250} * \\
L_{1500} *, L_{\infty} * \\
L_{250}\end{array}$ \\
\hline$D=10$ & $\begin{array}{l}L_{K}> \\
L_{K}= \\
L_{K}<\end{array}$ & $\begin{array}{l}L_{0} *, L_{100} *, L_{250} *, L_{500} *, L_{750} *, L_{1000} * \\
L_{1250} *, L_{1500} *, L_{\infty} *\end{array}$ & $\begin{array}{l}L_{K}> \\
L_{K}= \\
L_{K}<\end{array}$ & $\begin{array}{l}L_{0} *, L_{100} *, L_{500} *, L_{750} *, L_{1000} *, L_{1250} * \\
L_{1500} *, L_{\infty} * \\
L_{250}\end{array}$ & $\begin{array}{l}L_{K}> \\
L_{K}= \\
L_{K}<\end{array}$ & $\begin{array}{l}L_{0} *, L_{100} *, L_{250} *, L_{750} *, L_{1000} *, L_{1250} * \\
L_{1500} *, L_{\infty} * \\
L_{500}\end{array}$ \\
\hline$D=30$ & $\begin{array}{l}L_{K}> \\
L_{K}= \\
L_{K}<\end{array}$ & $\begin{array}{l}L_{0} *, L_{100} *, L_{250} *, L_{500} *, L_{750} *, L_{1000} * \\
L_{1250} *, L_{1500} *, L_{\infty} *\end{array}$ & $\begin{array}{l}L_{K}> \\
L_{K}= \\
L_{K}<\end{array}$ & $\begin{array}{l}L_{0} *, L_{100} *, L_{250} *, L_{500} *, L_{1000} *, L_{1250} * \\
L_{1500} *, L_{\infty} * \\
L_{750}\end{array}$ & $\begin{array}{l}L_{K}> \\
L_{K}= \\
L_{K}<\end{array}$ & $\begin{array}{l}L_{0} *, L_{100} *, L_{250} *, L_{500} *, L_{750} *, L_{1000} * \\
L_{1250} *, L_{\infty} * \\
L_{1500}\end{array}$ \\
\hline$D=50$ & $\begin{array}{l}L_{K}> \\
L_{K}= \\
L_{K}<\end{array}$ & $\begin{array}{l}L_{0} *, L_{100} *, L_{250} *, L_{500} *, L_{750} *, L_{1000} * \\
L_{1250} *, L_{1500} *, L_{\infty} *\end{array}$ & $\begin{array}{l}L_{K}> \\
L_{K}= \\
L_{K}<\end{array}$ & $\begin{array}{l}L_{0} *, L_{100} *, L_{250} *, L_{500} *, L_{750} *, L_{1000} * \\
L_{1500} *, L_{\infty} * \\
L_{1250}\end{array}$ & $\begin{array}{l}L_{K}> \\
L_{K}= \\
L_{K}<\end{array}$ & $\begin{array}{l}L_{0} *, L_{100} *, L_{250} *, L_{500} *, L_{750} *, L_{1000} *, \\
L_{1250} *, L_{1500} *, L_{\infty} *\end{array}$ \\
\hline
\end{tabular}

\section{Table 11}

$f_{2}$, vertical approach, MaxFEs $=250,000$, NHST

\begin{tabular}{|c|c|c|c|c|c|c|}
\hline & & $\mathrm{SN}=24$ & & $\mathrm{SN}=50$ & & $\mathrm{SN}=100$ \\
\hline$D=2$ & $\begin{array}{l}L_{K}> \\
L_{K}= \\
L_{K}<\end{array}$ & $\begin{array}{l}L_{0} * \\
L_{100}, L_{250}, L_{500}, L_{750}, L_{1000}, L_{1250}, \\
L_{1500}, L_{\infty}\end{array}$ & $\begin{array}{l}L_{K}> \\
L_{K}= \\
L_{K}<\end{array}$ & $\begin{array}{l}L_{0} * \\
L_{100}, L_{250}, L_{500}, L_{750}, L_{1000}, L_{1250}, \\
L_{1500}, L_{\infty}\end{array}$ & $\begin{array}{l}L_{K}> \\
L_{K}= \\
L_{K}<\end{array}$ & $\begin{array}{l}L_{0} * \\
L_{100}, L_{250}, L_{500}, L_{750}, L_{1000}, L_{1250}, \\
L_{1500}, L_{\infty}\end{array}$ \\
\hline$D=5$ & $\begin{array}{l}L_{K}> \\
L_{K}= \\
L_{K}<\end{array}$ & $\begin{array}{l}L_{0} *, L_{\infty} \\
L_{100}, L_{250}, L_{500}, L_{750}, L_{1000}, L_{1250}, \\
L_{1500}\end{array}$ & $\begin{array}{l}L_{K}> \\
L_{K}= \\
L_{K}<\end{array}$ & $\begin{array}{l}L_{0} *, L_{\infty} \\
L_{100}, L_{250}, L_{500}, L_{750}, L_{1000}, L_{1250}, \\
L_{1500}\end{array}$ & $\begin{array}{l}L_{K}> \\
L_{K}= \\
L_{K}<\end{array}$ & $\begin{array}{l}L_{0} *, L_{\infty} \\
L_{100}, L_{250}, L_{500}, L_{750}, L_{1000}, L_{1250}, \\
L_{1500}\end{array}$ \\
\hline$D=10$ & $\begin{array}{l}L_{K}> \\
L_{K}= \\
L_{K}<\end{array}$ & $\begin{array}{l}L_{0} *, L_{100} *, L_{250} *, L_{500} *, L_{750} *, L_{1000} * \\
L_{1250} *, L_{1500} *, L_{\infty} *\end{array}$ & $\begin{array}{l}L_{K}> \\
L_{K}= \\
L_{K}<\end{array}$ & $\begin{array}{l}L_{0} *, L_{100} *, L_{500} *, L_{750} *, L_{1000} *, L_{1250} * \\
L_{1500} *, L_{\infty} * \\
L_{250}\end{array}$ & $\begin{array}{l}L_{K}> \\
L_{K}= \\
L_{K}<\end{array}$ & $\begin{array}{l}L_{0} *, L_{100} *, L_{250} *, L_{750} *, L_{1000} *, L_{1250} * \\
L_{1500}, L_{\infty} * \\
L_{500}\end{array}$ \\
\hline$D=30$ & $\begin{array}{l}L_{K}> \\
L_{K}= \\
L_{K}<\end{array}$ & $\begin{array}{l}L_{0} *, L_{100} *, L_{250} *, L_{500} *, L_{750} *, L_{1000} * \\
L_{1250} *, L_{1500} *, L_{\infty} *\end{array}$ & $\begin{array}{l}L_{K}> \\
L_{K}= \\
L_{K}<\end{array}$ & $\begin{array}{l}L_{0} *, L_{100} *, L_{250} *, L_{500} *, L_{1000} *, L_{1250} * \\
L_{1500} *, L_{\infty} * \\
L_{750}\end{array}$ & $\begin{array}{l}L_{K}> \\
L_{K}= \\
L_{K}<\end{array}$ & $\begin{array}{l}L_{0} *, L_{100} *, L_{250} *, L_{500} *, L_{750} *, L_{1000} * \\
L_{1250} *, L_{\infty} * \\
L_{1500}\end{array}$ \\
\hline$D=50$ & $\begin{array}{l}L_{K}> \\
L_{K}= \\
L_{K}<\end{array}$ & $\begin{array}{l}L_{0} *, L_{100} *, L_{250} *, L_{500} *, L_{750} *, L_{1000} * \\
L_{1250} *, L_{1500} *, L_{\infty} *\end{array}$ & $\begin{array}{l}L_{K}> \\
L_{K}= \\
L_{K}<\end{array}$ & $\begin{array}{l}L_{0} *, L_{100} *, L_{250} *, L_{500} *, L_{750} *, L_{1000} * \\
L_{1500} *, L_{\infty} * \\
L_{1250}\end{array}$ & $\begin{array}{l}L_{K}> \\
L_{K}= \\
L_{K}<\end{array}$ & $\begin{array}{l}L_{0} *, L_{100} *, L_{250} *, L_{500} *, L_{750} *, L_{1000} * \\
L_{1250} *, L_{1500} *, L_{\infty} *\end{array}$ \\
\hline
\end{tabular}


Table 12

$f_{3}$, vertical approach, MaxFEs $=250,000$, NHST

\begin{tabular}{|c|c|c|c|c|c|c|}
\hline & & $\mathrm{SN}=24$ & & $\mathrm{SN}=\mathbf{5 0}$ & & $\mathrm{SN}=100$ \\
\hline$D=2$ & $\begin{array}{l}L_{K}> \\
L_{K}= \\
L_{K}<\end{array}$ & $\begin{array}{l}L_{0} * \\
L_{100}, L_{250}, L_{500}, L_{750}, L_{1000}, L_{1250}, \\
L_{1500}, L_{\infty}\end{array}$ & $\begin{array}{l}L_{K}> \\
L_{K}= \\
L_{K}<\end{array}$ & $\begin{array}{l}L_{0 *} \\
L_{100}, L_{250}, L_{500}, L_{750}, L_{1000}, L_{1250}, \\
L_{1500}, L_{\infty}\end{array}$ & $\begin{array}{l}L_{K}> \\
L_{K}= \\
L_{K}<\end{array}$ & $\begin{array}{l}L_{0} * \\
L_{100}, L_{250}, L_{500}, L_{750}, L_{1000}, L_{1250}, \\
L_{1500}, L_{\infty}\end{array}$ \\
\hline$D=5$ & $\begin{array}{l}L_{K}> \\
L_{K}= \\
L_{K}<\end{array}$ & $\begin{array}{l}L_{0} * \\
L_{100}, L_{250}, L_{500}, L_{750}, L_{1000}, L_{1250}, \\
L_{1500}, L_{\infty}\end{array}$ & $\begin{array}{l}L_{K}> \\
L_{K}= \\
L_{K}<\end{array}$ & $\begin{array}{l}L_{0 *} \\
L_{100}, L_{250}, L_{500}, L_{750}, L_{1000}, L_{1250}, \\
L_{1500}, L_{\infty}\end{array}$ & $\begin{array}{l}L_{K}> \\
L_{K}= \\
L_{K}<\end{array}$ & $\begin{array}{l}L_{0} * \\
L_{100}, L_{250}, L_{500}, L_{750}, L_{1000}, L_{1250}, \\
L_{1500}, L_{\infty}\end{array}$ \\
\hline$D=10$ & $\begin{array}{l}L_{K}> \\
L_{K}= \\
L_{K}<\end{array}$ & $\begin{array}{l}L_{0} * \\
L_{100}, L_{250}, L_{500}, L_{750}, L_{1000}, L_{1250}, \\
L_{1500}, L_{\infty}\end{array}$ & $\begin{array}{l}L_{K}> \\
L_{K}= \\
L_{K}<\end{array}$ & $\begin{array}{l}L_{0} * \\
L_{100}, L_{250}, L_{500}, L_{750}, L_{1000}, L_{1250}, \\
L_{1500}, L_{\infty}\end{array}$ & $\begin{array}{l}L_{K}> \\
L_{K}= \\
L_{K}<\end{array}$ & $\begin{array}{l}L_{0} * \\
L_{100}, L_{250}, L_{500}, L_{750}, L_{1000}, L_{1250}, \\
L_{1500}, L_{\infty}\end{array}$ \\
\hline$D=30$ & $\begin{array}{l}L_{K}> \\
L_{K}= \\
L_{K}<\end{array}$ & $\begin{array}{l}L_{0} *, L_{100} * \\
L_{250}, L_{500}, L_{750}, L_{1000}, L_{1250}, L_{1500}, L_{\infty}\end{array}$ & $\begin{array}{l}L_{K}> \\
L_{K}= \\
L_{K}<\end{array}$ & $\begin{array}{l}L_{0} *, L_{100} * \\
L_{250}, L_{500}, L_{750}, L_{1000}, L_{1250}, L_{1500}, L_{\infty}\end{array}$ & $\begin{array}{l}L_{K}> \\
L_{K}= \\
L_{K}<\end{array}$ & $\begin{array}{l}L_{0} *, L_{100} * \\
L_{250}, L_{500}, L_{750}, L_{1000}, L_{1250}, L_{1500}, L_{\infty}\end{array}$ \\
\hline$D=50$ & $\begin{array}{l}L_{K}> \\
L_{K}= \\
L_{K}<\end{array}$ & $\begin{array}{l}L_{0} *, L_{100} *, L_{250} *, L_{500} *, L_{750} *, L_{1000} * \\
L_{1250} *, L_{1500} *, L_{\infty} *\end{array}$ & $\begin{array}{l}L_{K}> \\
L_{K}= \\
L_{K}<\end{array}$ & $\begin{array}{l}L_{0} *, L_{100} *, L_{250} *, L_{500} *, L_{750} *, L_{1000} * \\
L_{1500} *, L_{\infty} * \\
L_{1250}\end{array}$ & $\begin{array}{l}L_{K}> \\
L_{K}= \\
L_{K}<\end{array}$ & $\begin{array}{l}L_{0} *, L_{100} *, L_{250} *, L_{500} *, L_{750} *, L_{1000} * \\
L_{1250} *, L_{1500} *, L_{\infty} *\end{array}$ \\
\hline
\end{tabular}

Table 13

$f_{4}$, vertical approach, MaxFEs $=250,000$, NHST

\begin{tabular}{|c|c|c|c|c|c|c|}
\hline & & $\mathrm{SN}=24$ & & $\mathrm{SN}=50$ & & $\mathrm{SN}=100$ \\
\hline$D=2$ & $\begin{array}{l}L_{K}> \\
L_{K}= \\
L_{K}<\end{array}$ & $\begin{array}{l}L_{0} *, L_{100} *, L_{250} *, L_{500} *, L_{750} *, L_{1000} * \\
L_{1250} *, L_{1500} *, L_{\infty} *\end{array}$ & $\begin{array}{l}L_{K}> \\
L_{K}= \\
L_{K}<\end{array}$ & $\begin{array}{l}L_{0} *, L_{100} *, L_{250} *, L_{500} *, L_{750} *, L_{1000} * \\
L_{1250} *, L_{1500} *, L_{\infty} *\end{array}$ & $\begin{array}{l}L_{K}> \\
L_{K}= \\
L_{K}<\end{array}$ & $\begin{array}{l}L_{0} *, L_{250} *, L_{500} *, L_{750} *, L_{1000} *, L_{1250} * \\
L_{1500} *, L_{\infty} * \\
L_{100}\end{array}$ \\
\hline$D=5$ & $\begin{array}{l}L_{K}> \\
L_{K}= \\
L_{K}<\end{array}$ & $\begin{array}{l}L_{0} *, L_{100} *, L_{250}, L_{500}, L_{1000}, L_{\infty} \\
L_{750}, L_{1250}, L_{1500}\end{array}$ & $\begin{array}{l}L_{K}> \\
L_{K}= \\
L_{K}<\end{array}$ & $\begin{array}{l}L_{0} *, L_{100} *, L_{250} *, L_{500}, L_{750}, L_{1000}, \\
L_{1250}, L_{1500}, L_{\infty}\end{array}$ & $\begin{array}{l}L_{K}> \\
L_{K}= \\
L_{K}<\end{array}$ & $\begin{array}{l}L_{0} *, L_{100} *, L_{500}, L_{750}, L_{1000}, L_{1250}, \\
L_{1500}, L_{\infty} \\
L_{250}\end{array}$ \\
\hline$D=10$ & $\begin{array}{l}L_{K}> \\
L_{K}= \\
L_{K}<\end{array}$ & $\begin{array}{l}L_{0} *, L_{100} *, L_{250} *, L_{500}, L_{750}, L_{1250} \\
L_{1000}, L_{\infty} \\
L_{1500}\end{array}$ & $\begin{array}{l}L_{K}> \\
L_{K}= \\
L_{K}<\end{array}$ & $\begin{array}{l}L_{0} *, L_{100} *, L_{500}, L_{750}, L_{1000}, L_{1250}, \\
L_{1500}, L_{\infty} \\
L_{250}\end{array}$ & $\begin{array}{l}L_{K}> \\
L_{K}= \\
L_{K}<\end{array}$ & $\begin{array}{l}L_{0} *, L_{100} *, L_{250} *, L_{750} *, L_{1000} *, L_{1250} * \\
L_{1500} *, L_{\infty} * \\
L_{500}\end{array}$ \\
\hline$D=30$ & $\begin{array}{l}L_{K}> \\
L_{K}= \\
L_{K}<\end{array}$ & $\begin{array}{l}L_{0} *, L_{100} *, L_{250} *, L_{500} *, L_{750} *, L_{1000} * \\
L_{1250} *, L_{1500} *, L_{\infty} *\end{array}$ & $\begin{array}{l}L_{K}> \\
L_{K}= \\
L_{K}<\end{array}$ & $\begin{array}{l}L_{0} *, L_{100} *, L_{250} *, L_{500} *, L_{1000} *, L_{1250} * \\
L_{1500} *, L_{\infty} * \\
L_{750}\end{array}$ & $\begin{array}{l}L_{K}> \\
L_{K}= \\
L_{K}<\end{array}$ & $\begin{array}{l}L_{0} *, L_{100} *, L_{250} *, L_{500} *, L_{750} *, L_{1000} * \\
L_{1250} *, L_{\infty} * \\
L_{1500}\end{array}$ \\
\hline$D=50$ & $\begin{array}{l}L_{K}> \\
L_{K}= \\
L_{K}<\end{array}$ & $\begin{array}{l}L_{0} *, L_{100} *, L_{250} *, L_{500} *, L_{750} *, L_{1000} * \\
L_{1250} *, L_{1500} *, L_{\infty} *\end{array}$ & $\begin{array}{l}L_{K}> \\
L_{K}= \\
L_{K}<\end{array}$ & $\begin{array}{l}L_{0} *, L_{100} *, L_{250} *, L_{500} *, L_{750} *, L_{1000} * \\
L_{1500} *, L_{\infty} * \\
L_{1250}\end{array}$ & $\begin{array}{l}L_{K}> \\
L_{K}= \\
L_{K}<\end{array}$ & $\begin{array}{l}L_{0} *, L_{100} *, L_{250} *, L_{500} *, L_{750} *, L_{1000} * \\
L_{1250} *, L_{1500} *, L_{\infty} *\end{array}$ \\
\hline
\end{tabular}

Table 14

$f_{5}$, vertical approach, MaxFEs $=250,000$, NHST

\begin{tabular}{|c|c|c|c|c|c|c|}
\hline & & $\mathrm{SN}=24$ & & $\mathrm{SN}=\mathbf{5 0}$ & & $\mathrm{SN}=100$ \\
\hline & $L_{K}>$ & $\begin{array}{l}L_{0}, L_{100}, L_{250}, L_{500}, L_{750}, L_{1000}, L_{1250} \\
L_{1500}, L_{\infty} *\end{array}$ & $L_{K}>$ & $\begin{array}{l}L_{0}, L_{100}, L_{250}, L_{500}, L_{750}, L_{1000} *, L_{1250}, \\
L_{1500}, L_{\infty}\end{array}$ & $L_{K}>$ & $L_{250}, L_{750}, L_{1000}, L_{1250}, L_{1500} *, L_{\infty}$ \\
\hline \multirow[t]{2}{*}{$\mathrm{D}=2$} & $L_{K}=$ & & $L_{K}=$ & & $L_{K}=$ & $L_{100}$ \\
\hline & $L_{K}<$ & & $L_{K}<$ & & $L_{K}<$ & $L_{0}, L_{500}$ \\
\hline \multirow{3}{*}{$D=5$} & $L_{K}>$ & $L_{0} *$ & $L_{K}>$ & $L_{0} *, L_{100} *$ & $L_{K}>$ & $L_{0} *, L_{100} *$ \\
\hline & $L_{K}=$ & & $L_{K}=$ & & $L_{K}=$ & $L_{250}$ \\
\hline & $L_{K}<$ & $\begin{array}{l}L_{100} *, \quad L_{250} *, \quad L_{500} *, \quad L_{750} *, \quad L_{1000} * \\
L_{1250}, L_{1500}, L_{\infty} *\end{array}$ & $L_{K}<$ & $\begin{array}{l}L_{250}, L_{500 *}, L_{750 *}, L_{1000 *}, L_{1250 *}, \\
L_{1500} *, L_{\infty} *\end{array}$ & $L_{K}<$ & $L_{500}, L_{750}, L_{1000}, L_{1250}, L_{1500} *, L_{\infty}$ \\
\hline \multirow[t]{2}{*}{$D=10$} & $\begin{array}{l}L_{K}> \\
L_{K}=\end{array}$ & $L_{0} *, L_{100}$ & $\begin{array}{l}L_{K}> \\
L_{K}=\end{array}$ & $\begin{array}{l}L_{0} *, L_{100} *, L_{500} \\
L_{250}\end{array}$ & $\begin{array}{l}L_{K}> \\
L_{K}=\end{array}$ & $\begin{array}{l}L_{0} *, L_{100} *, L_{250}, L_{1000}, L_{1500}, L_{\infty} \\
L_{500}\end{array}$ \\
\hline & $L_{K}<$ & $\begin{array}{l}L_{250 *}, \quad L_{500 *}, L_{750} *, \quad L_{1000} *, \quad L_{1250 *}, \\
L_{1500 *,} L_{\infty} *\end{array}$ & $L_{K}<$ & $L_{750}, L_{1000}, L_{1250}, L_{1500}, L_{\infty}$ & $L_{K}<$ & $L_{750}, L_{1250}$ \\
\hline \multirow{3}{*}{$\mathbf{D}=\mathbf{3 0}$} & $L_{K}>$ & $L_{0} *, L_{100}, L_{1250}$ & $L_{K}>$ & $\begin{array}{l}L_{0} *, \quad L_{100}, L_{250}, L_{500}, L_{1000}, L_{1250}, \\
L_{1500}, L_{\infty}\end{array}$ & $L_{K}>$ & $L_{0} *, L_{250} L_{500}, L_{750}, L_{1250}, L_{\infty}$ \\
\hline & $L_{K}=$ & & $L_{K}=$ & $L_{750}$ & $L_{K}=$ & $L_{1500}$ \\
\hline & $L_{K}<$ & $L_{250}, L_{500}, L_{750}, L_{1000}, L_{1500}, L_{\infty}$ & $L_{K}<$ & & $L_{K}<$ & $L_{100}, L_{1000}$ \\
\hline \multirow[t]{2}{*}{$\mathrm{D}=\mathbf{5 0}$} & $\begin{array}{l}L_{K}> \\
L_{K}=\end{array}$ & $L_{0} *, L_{1250}$ & $\begin{array}{l}L_{K}> \\
L_{K}=\end{array}$ & $\begin{array}{l}L_{0} *, L_{250}, L_{1500} \\
L_{1250}\end{array}$ & $\begin{array}{l}L_{K}> \\
L_{K}=\end{array}$ & $L_{0} *$ \\
\hline & $L_{K}<$ & $L_{250}, L_{500}, L_{750}, L_{1000}, L_{1500}, L_{\infty}$ & $L_{K}<$ & $L_{100}, L_{500}, L_{750}, L_{1000}, L_{\infty}$ & $L_{K}<$ & $\begin{array}{l}L_{100}, L_{250}, L_{500}, L_{750}, L_{1000}, L_{1250}, \\
L_{1500}, L_{\infty}\end{array}$ \\
\hline
\end{tabular}


On the other hand, for $f_{1}$ and $f_{4}, L_{k}$ was never the absolute best value, meaning that there was always a better or at least equal 'limit' value. For $f_{2}, f_{3}$, and $f_{5}$ that was not the case, as $L_{k}$ was in some cases better than all 9 fixed 'limit' values. In particular, for $f_{2}: S N=24$ and $D=2, S N$ $=50$ and $D=2$, and $S N=50$ and $D=5$. For $f_{3}: S N=24$ and $D=10$. For $f_{5}: S N=24$ and $D=2$, and $S N=50$ and $D=2$. This, however, does not mean that the 'limit' values that could be better than $L_{k}$ for these problems and settings do not exist; it only means that $L_{k}$ was better for these problems and settings than these 9 fixed 'limit' values.

\subsubsection{Experiment 2: Vertical Approach with $M a x F E s=250,000$}

In this section, there were more fitness evaluations available, and $L_{k}$ was almost always better than or equal to other settings. This means that when large enough fitness evaluations were available, $L_{k}$ was an appropriate choice regardless of the population size and dimension of a problem (for the benchmark suite under investigation). Only for problem $f_{5}$, which is harder than the other four problems, $L_{k}$ was in two cases worse than some other settings. Firstly for $S N=24$ and $D=5$ and secondly for $S N=24$ and $D=10$. These differences, however, were never significant. All the differences are shown in Tables 10-14. These findings suggest that setting a control parameter 'limit' depends on the available maximum number of fitness evaluations.

\subsubsection{Experiment 3: Horizontal Approach $-10^{-6}$ MaxFEs $=1,000,000$}

During the horizontal approach where we measured the number of function evaluations needed to reach a (sub-)optimal solution, $L_{k}$ had the better alternatives in almost all cases. For $f_{1}$, these better alternatives were available for the small population size $S N=24$ and for the bigger population size $S N=100$, whereas for $S N=$ $50, L_{k}$ was worse only for $D=5$ and better for all other dimension values. For $f_{2}, L_{k}$ was worse than all the population sizes and dimension values, except for $S N=50$ and $D=10, S N=100$ and $D=10, S N=100$ and $D=50$, and $S N=100$ and $D=50$. For $f_{3}$ and $f_{4}, L_{k}$ always had a better alternative and was always worse than at least one other 'limit' value, regardless of the population size and dimension of a problem. Lastly, for $f_{5}$ and small dimension $D=2$ (and any population size values), $L_{k}$ had better alternatives, whilst for other dimensions and population sizes all 'limit' values performed the same. This happened due to the fact that none of these 'limit' values had found the (sub-)optimal solution $10^{-6}$ after
1,000,000 fitness evaluations. For $D=2$, some 'limit' values found (sub-)optimal solutions during some runs, and therefore they performed better than $L_{k}$. Whilst there were a lot of differences found between $L_{k}$ and other 'limit' values, these differences were rarely significant. There were only two problems for which $L_{k}$ was significantly worse than some other 'limit' values. First was $f_{1}$ when $L_{k}$ was significantly worse for small population size $S N=24$ for all dimensions. The other was $f_{4}$ when $L_{k}$ was significantly worse than all other 'limit' values except $L_{0}$ for small population size $S N=24$ and small dimension $D=2$. These differences are shown in Tables 15-19.

\subsubsection{Experiment 4: Horizontal Approach $10^{-12}$ MaxFEs $=1,000,000$}

In this section, the (sub-)optimal solution was set at $10^{-12}$, which was a harder problem than finding (sub-) optimal solution $10^{-6}$. Again, $L_{k}$ almost always had a better alternative. For $f_{1}$ better 'limit' values were found for small population size $S N=24$ regardless of dimension $D$ and for the bigger population size $S N$ $=100$ where the dimension was greater than $D=2$, whilst for $S N=50, L_{k}$ had better alternatives for small dimensions $D=2$ and $D=5$ and bigger dimension $D=$ 50 . For $f_{2}, L_{k}$ had better alternatives regardless of the population size and dimension values. The same went for $f_{3}$, except when the population size was $S N=50$ and dimension $D=5$, where $L_{k}$ was better than the other fixed 'limit' values. For $f_{4}, L_{k}$ was better than the other fixed 'limit' values when dimension $D=30$, whilst for other dimensions (regardless of population size value) there were better alternatives. For $f_{5}$, all 'limit' values were the same during performances, which was due to the fact that none of them found the (sub-) optimal solution $10^{-12}$ after 1,000,000 fitness evaluations. These differences are shown in Tables 20-24.

\subsubsection{Experiment 5: Large Dimensions}

In this section, the horizontal approach with (sub-)optimal solution set at $10^{-6}$ was repeated for larger dimensions, $D=\{100,200,300\}$, since we have expected that the recommended formulae might perform even worse for large dimensions (such very large optimisation problems are now common for some benchmark suites [32]). Again, fixed 'limit' values, $\mathrm{L}=\{0,1000,2000$, $3000,4000,5000,6000,7000,8000,9000,10000$, $11000,12000,13000,14000,15000, \infty\}$ were compared to Karaboga's setting $L_{k}$. Found differences are shown in Tables 25-29. As in previous four experiments, this 
Table 15

$f_{1}$, horizontal approach, $10^{-6}$, NHST

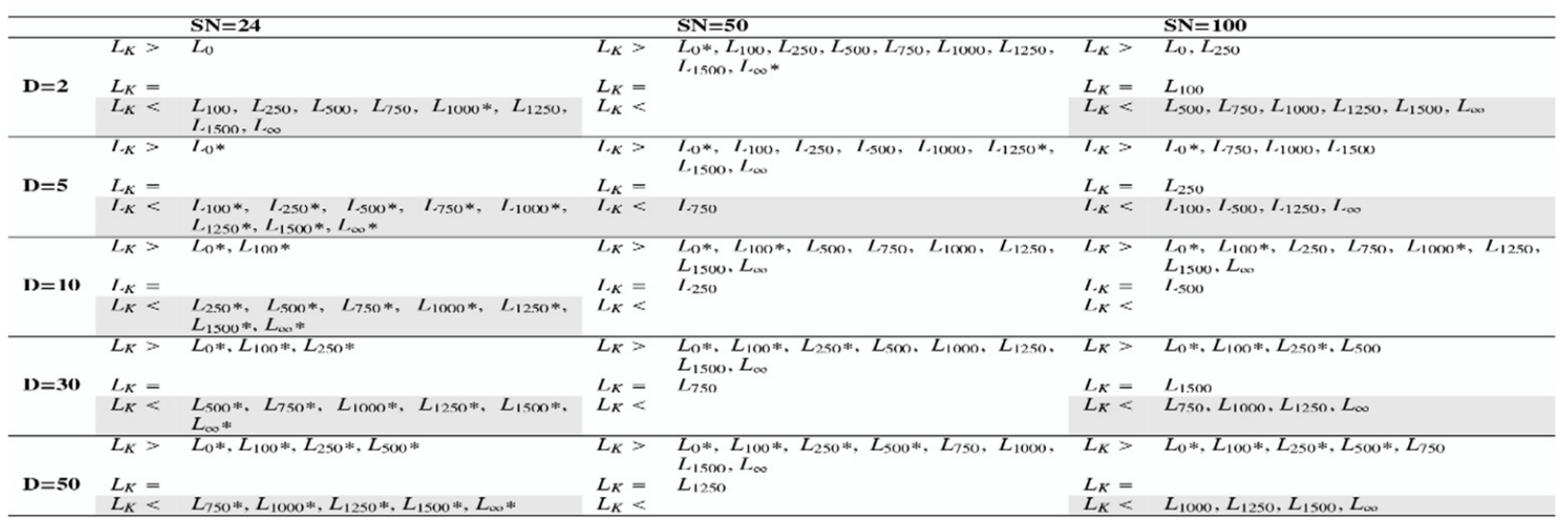

\section{Table 16}

$f_{2}$, horizontal approach, $10^{-6}$, NHST

\begin{tabular}{|c|c|c|c|c|c|c|}
\hline & & $\mathrm{SN}=24$ & & $\mathrm{SN}=\mathbf{5 0}$ & & $\mathrm{SN}=100$ \\
\hline \multirow{3}{*}{$D=2$} & $L_{K}>$ & $L_{0} *, L_{250}, L_{750}, L_{1000}, L_{1250}, L_{\infty}$ & $L_{K}>$ & $L_{0} *, L_{500}, L_{750}, L_{1000}, L_{1250}, L_{1500}, L_{\infty}$ & $L_{K}>$ & $L_{0} *, L_{250}$ \\
\hline & $L_{K}=$ & & $L_{K}=$ & & $L_{K}=$ & $L_{100}$ \\
\hline & $L_{K}<$ & $L_{100}, L_{500}, L_{1500}$ & $L_{K}<$ & $L_{100}, L_{250}$ & $L_{K}<$ & $L_{500}, L_{750}, L_{1000}, L_{1250}, L_{1500}, L_{\infty}$ \\
\hline \multirow{3}{*}{$\mathrm{D}=5$} & $L_{K}>$ & $L_{0} *, L_{750}$ & $L_{K}>$ & $L_{0} *, L_{1000}$ & $L_{K}>$ & $L_{0} *, L_{500}, L_{1000}, L_{1250}$ \\
\hline & $L_{K}=$ & & $L_{K}=$ & & $L_{K}=$ & $L_{250}$ \\
\hline & $L_{K}<$ & $L_{100}, L_{250}, L_{500}, L_{1000}, L_{1250}, L_{1500}, L_{\infty}$ & $L_{K}<$ & $L_{100}, L_{250}, L_{500}, L_{750}, L_{1250}, L_{1500}, L_{\infty}$ & $L_{K}<$ & $L_{100}, L_{750}, L_{1500}, L_{\infty}$ \\
\hline \multirow{2}{*}{$\mathrm{D}=\mathbf{1 0}$} & $L_{K}>$ & $L_{0} *, L_{250}, L_{750}$ & $L_{K}>$ & $\begin{array}{l}L_{0} *, L_{100}, L_{500}, L_{750}, L_{1000}, L_{1250}, \\
L_{1500}, L_{\infty}\end{array}$ & $L_{K}>$ & $\begin{array}{l}L_{0} *, L_{100}, L_{250}, L_{750}, L_{1000}, L_{1250}, \\
L_{1500}, L_{\infty}\end{array}$ \\
\hline & $L_{K}<$ & $L_{100}, L_{500}, L_{1000}, L_{1250}, L_{1500}, L_{\infty}$ & $L_{K}<$ & & $L_{K}<$ & \\
\hline \multirow{3}{*}{$\mathrm{D}=\mathbf{3 0}$} & $L_{K}>$ & $L_{0} *, L_{500}, L_{750}$ & $L_{K}>$ & $L_{0} *, L_{100}, L_{500}, L_{1000}, L_{1500}$ & $L_{K}>$ & $L_{0} *, L_{750}, L_{1250}$ \\
\hline & $L_{K}=$ & & $L_{K}=$ & $L_{750}$ & $L_{K}=$ & $L_{1500}$ \\
\hline & $L_{K}<$ & $L_{100}, L_{250}, L_{1000}, L_{1250}, L_{1500}, L_{\infty}$ & $L_{K}<$ & $L_{250}, L_{1250}, L_{\infty}$ & $L_{K}<$ & $L_{100}, L_{250}, L_{500}, L_{1000}, L_{\infty}$ \\
\hline \multirow{3}{*}{$\mathrm{D}=\mathbf{5 0}$} & $L_{K}>$ & $L_{0} *, L_{1250}$ & $L_{K}>$ & $L_{0} *, L_{100}, L_{250}, L_{500}, L_{1000}, L_{1500}, L_{\infty}$ & $L_{K}>$ & $\begin{array}{l}L_{0} *, L_{100}, L_{250}, L_{500}, L_{750}, L_{1000}, L_{1250}, \\
L_{1500}, L_{\infty}\end{array}$ \\
\hline & $L_{K}=$ & & $L_{K}=$ & $L_{1250}$ & $L_{K}=$ & \\
\hline & $L_{K}<$ & $L_{100}, L_{250}, L_{500}, L_{750}, L_{1000}, L_{1500}, L_{\infty}$ & $L_{K}<$ & $L_{750}$ & $L_{K}<$ & \\
\hline
\end{tabular}

Table 17

$f_{3}$, horizontal approach, $10^{-6}$, NHST

\begin{tabular}{|c|c|c|c|c|c|c|}
\hline & & $\mathrm{SN}=\mathbf{2 4}$ & & $\mathrm{SN}=\mathbf{5 0}$ & & $\mathrm{SN}=100$ \\
\hline & $L_{K}>$ & $\begin{array}{l}L_{0} *, L_{100}, L_{250}, L_{500}, L_{750}, L_{1000}, L_{1500} \\
L_{\infty}\end{array}$ & $L_{K}>$ & $L_{0} *, L_{100}$ & $L_{K}>$ & $L_{0} *$ \\
\hline \multirow[t]{2}{*}{$\mathrm{D}=\mathbf{2}$} & $L_{K}=$ & & $L_{K}=$ & & $L_{K}=$ & $L_{100}$ \\
\hline & $L_{K}<$ & $L_{1250}$ & $L_{K}<$ & $L_{250}, L_{500}, L_{750}, L_{1000}, L_{1250}, L_{1500}, L_{\infty}$ & $L_{K}<$ & $L_{250}, L_{500}, L_{750}, L_{1000}, L_{1250}, L_{1500}, L_{\infty}$ \\
\hline \multirow[t]{2}{*}{$D=5$} & $\begin{array}{l}L_{K}> \\
L_{K}=\end{array}$ & $L_{0} *, L_{100}, L_{500}, L_{750}, L_{1000}, L_{1500}$ & $\begin{array}{l}L_{K}> \\
L_{K}=\end{array}$ & $L_{0} *, L_{100}, L_{250}, L_{750}, L_{1000}, L_{1250}, L_{1500}$ & $\begin{array}{l}L_{K}> \\
L_{K}=\end{array}$ & $\begin{array}{l}L_{0} *, L_{1000}, L_{1250}, L_{1500}, L_{\infty} \\
L_{250}\end{array}$ \\
\hline & $L_{K}<$ & $L_{250}, L_{1250}, L_{\infty}$ & $L_{K}<$ & $L_{500}, L_{\infty}$ & $L_{K}<$ & $L_{100}, L_{500}, L_{750}$ \\
\hline $\mathrm{D}=\mathbf{1 0}$ & $\begin{array}{l}L_{K}> \\
L_{K}= \\
L_{K}<\end{array}$ & $\begin{array}{l}L_{0} *, L_{100}, L_{750}, L_{1000}, L_{1500} \\
L_{250}, L_{500}, L_{1250}, L_{\infty}\end{array}$ & $\begin{array}{l}L_{K}> \\
L_{K}= \\
L_{K}<\end{array}$ & $\begin{array}{l}L_{0} *, L_{100}, L_{500}, L_{750}, L_{1500}, L_{\infty} \\
L_{250} \\
L_{1000}, L_{1250}\end{array}$ & $\begin{array}{l}L_{K}> \\
L_{K}= \\
L_{K}<\end{array}$ & $\begin{array}{l}L_{0} *, L_{250}, L_{1500} \\
L_{500}\end{array}$ \\
\hline $\mathbf{D}=\mathbf{3 0}$ & $\begin{array}{l}L_{K}> \\
L_{K}> \\
L_{K}=\end{array}$ & $\begin{array}{l}L_{250}, L_{500}, L_{1250}, L_{\infty} \\
L_{0}, L_{100}, L_{250}, L_{500}, L_{750}, L_{1000}, L_{\infty} \\
L_{1250}, L_{1500}\end{array}$ & $\begin{array}{l}L_{K}< \\
L_{K}> \\
L_{K}= \\
L_{K}<\end{array}$ & $\begin{array}{l}L_{1000}, L_{1250} \\
L_{0} *, L_{500} \\
L_{750} \\
L_{100}, L_{250}, L_{1000}, L_{1250}, L_{1500}, L_{\infty}\end{array}$ & $\begin{array}{l}L_{K}< \\
L_{K}> \\
L_{K}= \\
L_{K}<\end{array}$ & $\begin{array}{l}L_{100}, L_{750}, L_{1000}, L_{1250}, L_{\infty} \\
L_{0} *, L_{500}, L_{1250} \\
L_{1500}\end{array}$ \\
\hline \multirow{3}{*}{$\mathrm{D}=\mathbf{5 0}$} & $L_{K}>$ & $\begin{array}{l}L_{0} *, L_{250}, L_{500}, L_{750}, L_{1000}, L_{1250} \\
L_{1500}, L_{\infty}\end{array}$ & $\begin{array}{l}L_{K}< \\
L_{K}>\end{array}$ & $\begin{array}{l}L_{100}, L_{250}, L_{1000}, L_{1250}, L_{1500}, L_{\infty} \\
L_{0} *, L_{100}, L_{500}\end{array}$ & $\begin{array}{l}L_{K}< \\
L_{K}>\end{array}$ & $\frac{L_{100}, L_{250}, L_{750}, L_{1000}, L_{\infty}}{L_{0} *, L_{100}, L_{250}, L_{750}, L_{1000}}$ \\
\hline & $L_{K}=$ & & $L_{K}=$ & $L_{1250}$ & $L_{K}=$ & \\
\hline & $L_{K}<$ & $L_{100}$ & $L_{K}<$ & $L_{250}, L_{750}, L_{1000}, L_{1500}, L_{\infty}$ & $L_{K}<$ & $L_{500}, L_{1250}, L_{1500}, L_{\infty}$ \\
\hline
\end{tabular}




\section{Table 18}

$f_{4}$, horizontal approach, $10^{-6}$, NHST

\begin{tabular}{|c|c|c|c|c|c|c|}
\hline & & $\mathrm{SN}=24$ & & $\mathrm{SN}=50$ & & $\mathrm{SN}=100$ \\
\hline \multirow[t]{2}{*}{$\mathrm{D}=2$} & $\begin{array}{l}L_{K}> \\
L_{K}=\end{array}$ & $L_{0} *$ & $\begin{array}{l}L_{K}> \\
L_{K}=\end{array}$ & $L_{0} *, L_{1000}$ & $\begin{array}{l}L_{K}> \\
L_{K}=\end{array}$ & $\begin{array}{l}L_{0} *, L_{250}, L_{750}, L_{1500}, L_{\infty} \\
L_{100}\end{array}$ \\
\hline & $L_{K}<$ & $\begin{array}{l}L_{100 *}, \quad L_{250 *}, \quad L_{500 *}, L_{750} *, L_{1000 *}, \\
L_{1250 *,} L_{1500^{*},} L_{\infty} *\end{array}$ & $L_{K}<$ & $L_{100}, L_{250}, L_{500}, L_{750}, L_{1250}, L_{1500}, L_{\infty}$ & $L_{K}<$ & $L_{500}, L_{1000}, L_{1250}$ \\
\hline$D=5$ & $\begin{array}{l}L_{K}> \\
L_{K}= \\
L_{K}<\end{array}$ & $\begin{array}{l}L_{0} * L_{100}, L_{500}, L_{1500}, L_{\infty} \\
L_{250}, L_{750}, L_{1000}, L_{1250}\end{array}$ & $\begin{array}{l}L_{K}> \\
L_{K}= \\
L_{K}<\end{array}$ & $\begin{array}{l}L_{0} *, L_{100}, L_{250}, L_{1000}, L_{1250}, L_{1500} \\
L_{500}, L_{750}, L_{\infty}\end{array}$ & $\begin{array}{l}L_{K}> \\
L_{K}= \\
L_{K}<\end{array}$ & $\begin{array}{l}L_{0} *, L_{100}, L_{500}, L_{750}, L_{1000}, L_{1250}, L_{\infty} \\
L_{250} \\
L_{1500}\end{array}$ \\
\hline$D=10$ & $\begin{array}{l}L_{K}> \\
L_{K}= \\
L_{K}<\end{array}$ & $\begin{array}{l}L_{0} *, L_{100}, L_{250}, L_{1000}, L_{1500}, L_{\infty} \\
L_{500}, L_{750}, L_{1250}\end{array}$ & $\begin{array}{l}L_{K}> \\
L_{K}= \\
L_{K}<\end{array}$ & $\begin{array}{l}L_{500}, L_{150}, L_{\infty} \\
L_{250} \\
L_{100}, L_{500}, L_{750}, L_{1500}, L_{\infty}\end{array}$ & $\begin{array}{l}L_{K}> \\
L_{K}> \\
L_{K}= \\
L_{K}<\end{array}$ & $\begin{array}{l}L_{1500} \\
L_{0} *, L_{250}, L_{750}, L_{1000}, L_{1500}, L_{\infty} \\
L_{500} \\
L_{100}, L_{1250}\end{array}$ \\
\hline \multirow[t]{2}{*}{$D=30$} & $\begin{array}{l}L_{K}> \\
L_{K}= \\
L_{K}<\end{array}$ & $\begin{array}{l}L_{0} *, L_{750} \\
L_{100}, L_{250}, L_{500}, L_{1000}, L_{1250}, L_{1500}, L_{\infty}\end{array}$ & $\begin{array}{l}L_{K}> \\
L_{K}= \\
L_{K}<\end{array}$ & $\begin{array}{l}L_{0} *, L_{250}, L_{500}, L_{1000}, L_{1500} \\
L_{750} \\
L_{100}, L_{1250}, L_{\infty}\end{array}$ & $\begin{array}{l}L_{K}> \\
L_{K}= \\
L_{K}<\end{array}$ & $\begin{array}{l}L_{0} *, L_{100}, L_{500}, L_{750}, L_{1250} \\
L_{1500} \\
L_{250}, L_{1000}, L_{\infty}\end{array}$ \\
\hline & $L_{K}>$ & $\begin{array}{l}L_{0} *, L_{100}, L_{250}, L_{500}, L_{750}, L_{1000}, L_{1250}, \\
L_{\infty}\end{array}$ & $L_{K}>$ & $L_{0} *, L_{\infty}$ & $L_{K}>$ & $L_{0} *, L_{100}, L_{250}, L_{500}, L_{1250}$ \\
\hline $\mathrm{D}=\mathbf{5 0}$ & $\begin{array}{l}L_{K}= \\
L_{K}<\end{array}$ & $L_{1500}$ & $\begin{array}{l}L_{K}= \\
L_{K}<\end{array}$ & $\begin{array}{l}L_{1250} \\
L_{100}, L_{250}, L_{500}, L_{750}, L_{1000}, L_{1500},\end{array}$ & $\begin{array}{l}L_{K}= \\
L_{K}<\end{array}$ & $L_{750}, L_{1000}, L_{1500}, L_{\infty}$ \\
\hline
\end{tabular}

Table 19

$f_{5}$, horizontal approach, $10^{-6}$, NHST

\begin{tabular}{|c|c|c|c|c|c|c|}
\hline & & $\mathrm{SN}=\mathbf{2 4}$ & & $\mathrm{SN}=\mathbf{5 0}$ & & $\mathrm{SN}=100$ \\
\hline \multirow[t]{2}{*}{$\mathrm{D}=2$} & $\begin{array}{l}L_{K}> \\
L_{K}=\end{array}$ & $\begin{array}{l}L_{0}, L_{100}, L_{250}, L_{500}, L_{750}, L_{1000}, L_{1250}, \\
L_{1500}\end{array}$ & $\begin{array}{l}L_{K}> \\
L_{K}=\end{array}$ & $L_{0}, L_{100}, L_{500}, L_{1250}, L_{1500}$ & $\begin{array}{l}L_{K}> \\
L_{K}=\end{array}$ & $L_{100}, L_{250}, L_{500}, L_{750}, L_{1250}, L_{1500}, L_{\infty}$ \\
\hline & $L_{K}<$ & $L_{\infty}$ & $L_{K}<$ & $L_{250}, L_{750}, L_{1000}, L_{\infty}$ & $L_{K}<$ & $L_{0}, L_{1000}$ \\
\hline$D=5$ & $\begin{array}{l}L_{K}> \\
L_{K}= \\
L_{K}<\end{array}$ & $\begin{array}{l}L_{0}, L_{100}, L_{250}, L_{500}, L_{750}, L_{1000}, L_{1250} \\
L_{1500}, L_{\infty}\end{array}$ & $\begin{array}{l}L_{K}> \\
L_{K}= \\
\\
L_{K}<\end{array}$ & $\begin{array}{l}L_{0}, L_{100}, L_{250}, L_{500}, L_{750}, L_{1000}, L_{1250} \\
L_{1500}, L_{\infty}\end{array}$ & $\begin{array}{l}L_{K}> \\
L_{K}= \\
L_{K}<\end{array}$ & $\begin{array}{l}L_{0}, L_{100}, L_{250}, L_{500}, L_{750}, L_{1000}, L_{1250} \\
L_{1500}, L_{\infty}\end{array}$ \\
\hline$D=10$ & $\begin{array}{l}L_{K}> \\
L_{K}= \\
\\
L_{K}<\end{array}$ & $\begin{array}{l}L_{0}, L_{100}, L_{250}, L_{500}, L_{750}, L_{1000}, L_{1250} \\
L_{1500}, L_{\infty}\end{array}$ & $\begin{array}{l}L_{K}> \\
L_{K}= \\
L_{K}<\end{array}$ & $\begin{array}{l}L_{0}, L_{100}, L_{250}, L_{500}, L_{750}, L_{1000}, L_{1250} \\
L_{1500}, L_{\infty}\end{array}$ & $\begin{array}{l}L_{K}> \\
L_{K}= \\
L_{K}<\end{array}$ & $\begin{array}{l}L_{0}, L_{100}, L_{250}, L_{500}, L_{750}, L_{1000}, L_{1250} \\
L_{1500}, L_{\infty}\end{array}$ \\
\hline $\mathrm{D}=\mathbf{3 0}$ & $\begin{array}{l}L_{K}> \\
L_{K}= \\
\\
L_{K}<\end{array}$ & $\begin{array}{l}L_{0}, L_{100}, L_{250}, L_{500}, L_{750}, L_{1000}, L_{1250} \\
L_{1500}, L_{\infty}\end{array}$ & $\begin{array}{l}L_{K}> \\
L_{K}= \\
\\
L_{K}<\end{array}$ & $\begin{array}{l}L_{0}, L_{100}, L_{250}, L_{500}, L_{750}, L_{1000}, L_{1250} \\
L_{1500}, L_{\infty}\end{array}$ & $\begin{array}{l}L_{K}> \\
L_{K}= \\
L_{K}<\end{array}$ & $\begin{array}{l}L_{0}, L_{100}, L_{250}, L_{500}, L_{750}, L_{1000}, L_{1250} \\
L_{1500}, L_{\infty}\end{array}$ \\
\hline $\mathrm{D}=\mathbf{5 0}$ & $\begin{array}{l}L_{K}> \\
L_{K}= \\
L_{K}<\end{array}$ & $\begin{array}{l}L_{0}, L_{100}, L_{250}, L_{500}, L_{750}, L_{1000}, L_{1250}, \\
L_{1500}, L_{\infty}\end{array}$ & $\begin{array}{l}L_{K}> \\
L_{K}= \\
L_{K}<\end{array}$ & $\begin{array}{l}L_{0}, L_{100}, L_{250}, L_{500}, L_{750}, L_{1000}, L_{1250} \\
L_{1500}, L_{\infty}\end{array}$ & $\begin{array}{l}L_{K}> \\
L_{K}= \\
L_{K}<\end{array}$ & $\begin{array}{l}L_{0}, L_{100}, L_{250}, L_{500}, L_{750}, L_{1000}, L_{1250} \\
L_{1500}, L_{\infty}\end{array}$ \\
\hline
\end{tabular}

Table 20

$f_{1}$, horizontal approach, $10^{-12}$, NHST

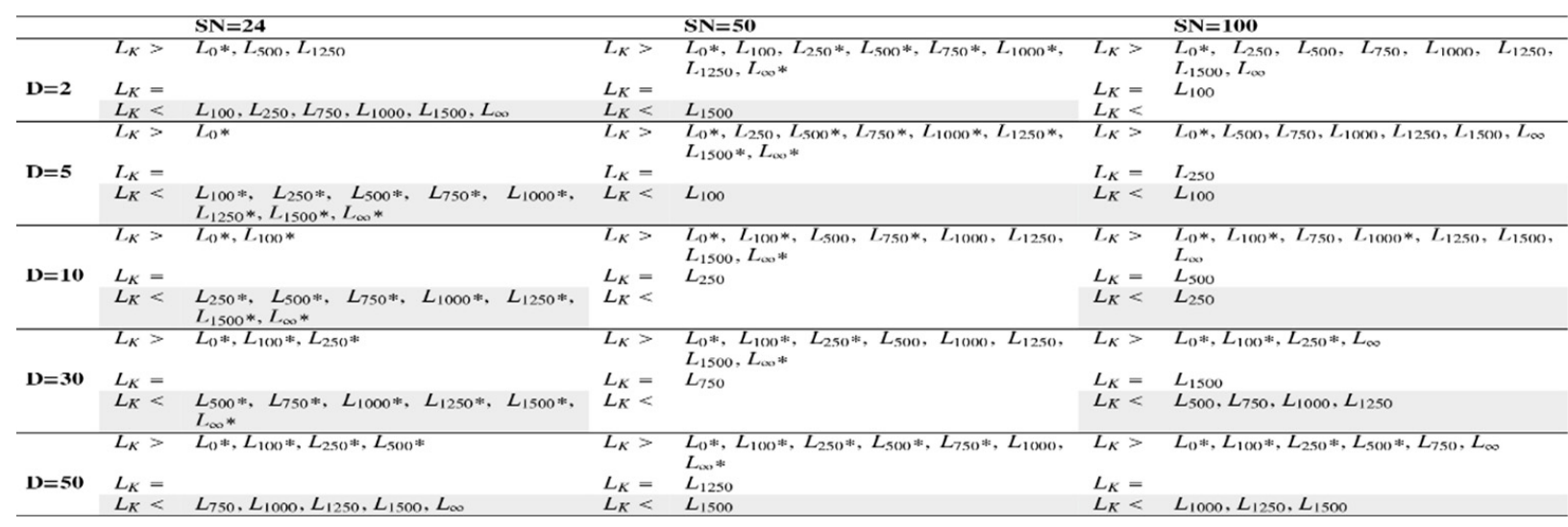


Table 21

$f_{2}$, horizontal approach, $10^{-12}$, NHST

\begin{tabular}{|c|c|c|c|c|c|c|}
\hline & & $\mathrm{SN}=24$ & & $\mathrm{SN}=50$ & & $\mathrm{SN}=100$ \\
\hline \multirow{3}{*}{$\mathrm{D}=2$} & $L_{K}>$ & $L_{0} *$ & $L_{K}>$ & $L_{0} *, L_{250}, L_{500}, L_{750}, L_{1000}, L_{1250}, L_{\infty}$ & $L_{K}>$ & $L_{0} *$ \\
\hline & $L_{K}=$ & & $L_{K}=$ & & $L_{K}=$ & $L_{100}$ \\
\hline & $L_{K}<$ & $\begin{array}{l}L_{100}, L_{250}, L_{500}, L_{750} *, L_{1000} *, L_{1250}, \\
L_{1500}, L_{\infty} *\end{array}$ & $L_{K}<$ & $L_{100}, L_{1500}$ & $L_{K}<$ & $L_{250}, L_{500}, L_{750}, L_{1000}, L_{1250}, L_{1500}, L_{\infty}$ \\
\hline \multirow{3}{*}{$D=5$} & $L_{K}>$ & $L_{0} *, L_{100}, L_{250}, L_{500}, L_{750}, L_{1250}, L_{\infty}$ & $L_{K}>$ & $L_{0} *, L_{500}, L_{750}$ & $L_{K}>$ & $L_{0} *, L_{1000}, L_{1500}$ \\
\hline & $L_{K}=$ & & $L_{K}=$ & & $L_{K}=$ & $L_{250}$ \\
\hline & $L_{K}<$ & $L_{1000}, L_{1500}$ & $L_{K}<$ & $L_{100}, L_{250}, L_{1000}, L_{1250}, L_{1500}, L_{\infty}$ & $L_{K}<$ & $L_{100}, L_{500}, L_{750}, L_{1250}, L_{\infty}$ \\
\hline \multirow{3}{*}{$D=10$} & $L_{K}>$ & $\begin{array}{l}L_{0} *, L_{100}, L_{250}, L_{500}, L_{750}, L_{1250}, L_{1500}, \\
L_{\infty}\end{array}$ & $L_{K}>$ & $L_{0} *, L_{100}, L_{500}, L_{750}, L_{1000}, L_{1500}, L_{\infty}$ & $L_{K}>$ & $L_{0} *, L_{250}, L_{750}, L_{1000}$ \\
\hline & $L_{K}=$ & & $L_{K}=$ & $L_{250}$ & $L_{K}=$ & $L_{500}$ \\
\hline & $L_{K}<$ & $L_{1000}$ & $L_{K}<$ & $L_{1250}$ & $L_{K}<$ & $L_{100}, L_{1250}, L_{1500}, L_{\infty}$ \\
\hline \multirow[t]{2}{*}{$\mathrm{D}=\mathbf{3 0}$} & $\begin{array}{l}L_{K}> \\
L_{K}=\end{array}$ & $L_{0} *, L_{250}, L_{750}, L_{1000}, L_{1500}, L_{\infty}$ & $\begin{array}{l}L_{K}> \\
L_{K}=\end{array}$ & $\begin{array}{l}L_{0} * \\
L_{750}\end{array}$ & $\begin{array}{l}L_{K}> \\
L_{K}=\end{array}$ & $\begin{array}{l}L_{0} *, L_{100}, L_{250}, L_{500}, L_{1000}, L_{1250}, L_{\infty} \\
L_{1500}\end{array}$ \\
\hline & $L_{K}<$ & $L_{100}, L_{500}, L_{1250}$ & $L_{K}<$ & $L_{100}, L_{250}, L_{500}, L_{1000}, L_{1250}, L_{1500}, L_{\infty}$ & $L_{K}<$ & $L_{750}$ \\
\hline \multirow[t]{2}{*}{$\mathrm{D}=\mathbf{5 0}$} & $\begin{array}{l}L_{K}> \\
L_{K}=\end{array}$ & $L_{0} *, L_{100}, L_{250}, L_{1000}, L_{1250}, L_{1500}$ & $\begin{array}{l}L_{K}> \\
L_{K}=\end{array}$ & $\begin{array}{l}L_{0} *, L_{100}, L_{250}, L_{500}, L_{750}, L_{1000}, L_{\infty} \\
L_{1250}\end{array}$ & $\begin{array}{l}L_{K}> \\
L_{K}=\end{array}$ & $L_{0} *, L_{250}$ \\
\hline & $L_{K}<$ & $L_{500}, L_{750}, L_{\infty}$ & $L_{K}<$ & $L_{1500}$ & $L_{K}<$ & $L_{100}, L_{500}, L_{750}, L_{1000}, L_{1250}, L_{1500}, L_{\infty}$ \\
\hline
\end{tabular}

Table 22

$f_{3}$, horizontal approach, $10^{-12}$, NHST

\begin{tabular}{|c|c|c|c|c|c|c|}
\hline & & $\mathrm{SN}=\mathbf{2 4}$ & & $\mathrm{SN}=50$ & & $\mathrm{SN}=100$ \\
\hline & $L_{K}>$ & $L_{0} *, L_{100}, L_{1000}, L_{1500}, L_{\infty}$ & $L_{K}>$ & $\begin{array}{l}L_{0} *, L_{100}, L_{250}, L_{500}, L_{750}, L_{1000}, L_{1250}, \\
L_{1500}\end{array}$ & $L_{K}>$ & $L_{0} *, L_{500}, L_{750}$ \\
\hline \multirow[t]{3}{*}{$D=2$} & $L_{K}=$ & & $L_{K}=$ & & $L_{K}=$ & $L_{100}$ \\
\hline & $L_{K}<$ & $L_{250}, L_{500}, L_{750}, L_{1250}$ & $L_{K}<$ & $L_{\infty}$ & $L_{K}<$ & $L_{250}, L_{1000}, L_{1250}, L_{1500}, L_{\infty}$ \\
\hline & $L_{K}>$ & $L_{0} *, L_{250}, L_{500}, L_{\infty}$ & $L_{K}>$ & $\begin{array}{l}L_{0} *, L_{100}, L_{250}, L_{500}, L_{750}, L_{1000}, L_{1250}, \\
L_{1500}, L_{\infty}\end{array}$ & $L_{K}>$ & $L_{0} *, L_{1250}, L_{\infty}$ \\
\hline \multirow[t]{2}{*}{$D=5$} & $L_{K}=$ & & $L_{K}=$ & & $L_{K}=$ & $L_{250}$ \\
\hline & $L_{K}<$ & $L_{100}, L_{750}, L_{1000}, L_{1250}, L_{1500}$ & $L_{K}<$ & & $L_{K}<$ & $L_{100}, L_{500}, L_{750}, L_{1000}, L_{1500}$ \\
\hline \multirow[t]{2}{*}{$\mathrm{D}=\mathbf{1 0}$} & $\begin{array}{l}L_{K}> \\
L_{K}=\end{array}$ & $L_{0} *, L_{100}, L_{750}, L_{1000}, L_{1250}, L_{1500}$ & $\begin{array}{l}L_{K}> \\
L_{K}=\end{array}$ & $\begin{array}{l}L_{0} *, L_{500} \\
L_{250}\end{array}$ & $\begin{array}{l}L_{K}> \\
L_{K}=\end{array}$ & $\begin{array}{l}L_{0} *, L_{100}, L_{1250}, L_{\infty} \\
L_{500}\end{array}$ \\
\hline & $L_{K}<$ & $L_{250}, L_{500}, L_{\infty}$ & $L_{K}<$ & $L_{100}, L_{750}, L_{1000}, L_{1250}, L_{1500}, L_{\infty}$ & $L_{K}<$ & $L_{250}, L_{750}, L_{1000}, L_{1500}$ \\
\hline \multirow[t]{2}{*}{$\mathrm{D}=\mathbf{3 0}$} & $\begin{array}{l}L_{K}> \\
L_{K}=\end{array}$ & $L_{0} *, L_{250}, L_{500}$ & $\begin{array}{l}L_{K}> \\
L_{K}=\end{array}$ & $\begin{array}{l}L_{0} *, L_{250}, L_{1250} \\
L_{750}\end{array}$ & $\begin{array}{l}L_{K}> \\
L_{K}=\end{array}$ & $\begin{array}{l}L_{0} *, L_{250}, L_{500}, L_{750}, L_{1250} \\
L_{1500}\end{array}$ \\
\hline & $L_{K}<$ & $L_{100}, L_{750}, L_{1000}, L_{1250}, L_{1500}, L_{\infty}$ & $L_{K}<$ & $L_{100}, L_{500}, L_{1000}, L_{1500}, L_{\infty}$ & $L_{K}<$ & $L_{100}, L_{1000}, L_{\infty}$ \\
\hline \multirow[t]{2}{*}{$\mathrm{D}=\mathbf{5 0}$} & $\begin{array}{l}L_{K}> \\
L_{K}=\end{array}$ & $L_{0} *, L_{500}, L_{750}, L_{\infty}$ & $\begin{array}{l}L_{K}> \\
L_{K}=\end{array}$ & $\begin{array}{l}L_{0} *, L_{750} \\
L_{1250}\end{array}$ & $\begin{array}{l}L_{K}> \\
L_{K}=\end{array}$ & $L_{0} *, L_{750}, L_{1000}, L_{1250}, L_{1500}, L_{\infty}$ \\
\hline & $L_{K}<$ & $L_{100}, L_{250}, L_{1000}, L_{1250}, L_{1500}$ & $L_{K}<$ & $L_{100}, L_{250}, L_{500}, L_{1000}, L_{1500}, L_{\infty}$ & $L_{K}<$ & $L_{100}, L_{250}, L_{500}$ \\
\hline
\end{tabular}

Table 23

$f_{4}$, horizontal approach, $10^{-12}$, NHST

\begin{tabular}{|c|c|c|c|c|c|c|}
\hline & & $\mathrm{SN}=24$ & & $\mathrm{SN}=50$ & & $\mathrm{SN}=100$ \\
\hline \multirow[t]{2}{*}{$\mathbf{D}=2$} & $\begin{array}{l}L_{K}> \\
L_{K}=\end{array}$ & $L_{0} *$ & $\begin{array}{l}L_{K}> \\
L_{K}=\end{array}$ & $L_{0} *, L_{100}, L_{250}, L_{500}, L_{1000}, L_{1250}, L_{\infty}$ & $\begin{array}{l}L_{K}> \\
L_{K}=\end{array}$ & $\begin{array}{l}L_{0} *, L_{250}, L_{500}, L_{750}, L_{1000}, L_{1500}, L_{\infty} \\
L_{100}\end{array}$ \\
\hline & $L_{K}<$ & $\begin{array}{l}L_{100} *, \quad L_{250} *, \quad L_{500} *, \quad L_{750} *, \quad L_{1000} *, \\
L_{1250 *,} L_{1500 *,} L_{\infty} *\end{array}$ & $L_{K}<$ & $L_{750}, L_{1500}$ & $L_{K}<$ & $L_{1250}$ \\
\hline \multirow[t]{2}{*}{$\mathbf{D}=\mathbf{5}$} & $\begin{array}{l}L_{K}> \\
L_{K}=\end{array}$ & $L_{0} *, L_{100}, L_{250}, L_{500}, L_{750}, L_{1000}, L_{1250}$ & $\begin{array}{l}L_{K}> \\
L_{K}=\end{array}$ & $L_{0} *, L_{100}, L_{750}, L_{1000}, L_{1500}, L_{\infty}$ & $\begin{array}{l}L_{K}> \\
L_{K}=\end{array}$ & $\begin{array}{l}L_{0} * \\
L_{250}\end{array}$ \\
\hline & $L_{K}<$ & $L_{1500}, L_{\infty}$ & $L_{K}<$ & $L_{250}, L_{500}, L_{1250}$ & $L_{K}<$ & $L_{100}, L_{500}, L_{750}, L_{1000}, L_{1250}, L_{1500}, L_{\infty}$ \\
\hline $\mathrm{D}=\mathbf{1 0}$ & $\begin{array}{l}L_{K}> \\
L_{K}= \\
L_{K}<\end{array}$ & $\begin{array}{l}L_{0} *, L_{100}, L_{500}, L_{1500} \\
L_{250}, L_{750}, L_{1000}, L_{1250}, L_{\infty}\end{array}$ & $\begin{array}{l}L_{K}> \\
L_{K}= \\
L_{K}<\end{array}$ & $\begin{array}{l}L_{0} * \\
L_{250} \\
L_{100}, L_{500}, L_{750}, L_{1000}, L_{1250}, L_{1500}, L_{\infty}\end{array}$ & $\begin{array}{l}L_{K}> \\
L_{K}= \\
L_{K}<\end{array}$ & $\begin{array}{l}L_{0} *, L_{100}, L_{750}, L_{1250} \\
L_{500} \\
L_{250}, L_{1000}, L_{1500}, L_{\infty}\end{array}$ \\
\hline $\mathbf{D}=\mathbf{3 0}$ & $\begin{array}{l}L_{K}> \\
L_{K}= \\
L_{K}<\end{array}$ & $\begin{array}{l}L_{0} *, L_{100}, L_{250}, L_{500} *, L_{750}, L_{1000} \\
L_{1250}, L_{1500}, L_{\infty}\end{array}$ & $\begin{array}{l}L_{K}> \\
L_{K}= \\
L_{K}<\end{array}$ & $\begin{array}{l}L_{0} *, L_{100}, L_{250}, L_{500}, L_{1000}, L_{1250}, \\
L_{1500}, L_{\infty} \\
L_{750}\end{array}$ & $\begin{array}{l}L_{K}> \\
L_{K}= \\
L_{K}<\end{array}$ & $\begin{array}{l}L_{0} *, L_{100}, L_{250}, L_{500}, L_{750}, L_{1000}, L_{1250}, \\
L_{\infty} \\
L_{1500}\end{array}$ \\
\hline $\mathrm{D}=\mathbf{5 0}$ & $\begin{array}{l}L_{K}> \\
L_{K}= \\
L_{K}<\end{array}$ & $\begin{array}{l}L_{0} *, L_{100}, L_{500}, L_{1000}, L_{1500} \\
L_{250}, L_{750}, L_{1250}, L_{\infty}\end{array}$ & $\begin{array}{l}L_{K}> \\
L_{K}= \\
L_{K}<\end{array}$ & $\begin{array}{l}L_{0} *, L_{100}, L_{500}, L_{1000}, L_{1500} \\
L_{1250} \\
L_{250}, L_{750}, L_{\infty}\end{array}$ & $\begin{array}{l}L_{K}> \\
L_{K}= \\
L_{K}<\end{array}$ & $\begin{array}{l}L_{0} *, L_{100} \\
L_{250}, L_{500}, L_{750}, L_{1000}, L_{1250}, L_{1500}, L_{\infty}\end{array}$ \\
\hline
\end{tabular}


Table 24

$f_{5}$, horizontal approach, $10^{-12}$, NHST

\begin{tabular}{|c|c|c|c|c|c|c|}
\hline & & $\mathrm{SN}=24$ & & $\mathrm{SN}=50$ & & $\mathrm{SN}=100$ \\
\hline$D=2$ & $\begin{array}{l}L_{K}> \\
L_{K}= \\
L_{K}<\end{array}$ & $\begin{array}{l}L_{0}, L_{100}, L_{250}, L_{500}, L_{750}, L_{1000}, L_{1250} \\
L_{1500}, L_{\infty}\end{array}$ & $\begin{array}{l}L_{K}> \\
L_{K}= \\
\\
L_{K}<\end{array}$ & $\begin{array}{l}L_{0}, L_{100}, L_{250}, L_{500}, L_{750}, L_{1000}, L_{1250} \\
L_{1500}, L_{\infty}\end{array}$ & $\begin{array}{l}L_{K}> \\
L_{K}= \\
L_{K}<\end{array}$ & $\begin{array}{l}L_{0}, L_{100}, L_{250}, L_{500}, L_{750}, L_{1000}, L_{1250}, \\
L_{1500}, L_{\infty}\end{array}$ \\
\hline$D=5$ & $\begin{array}{l}L_{K}> \\
L_{K}= \\
L_{K}<\end{array}$ & $\begin{array}{l}L_{0}, L_{100}, L_{250}, L_{500}, L_{750}, L_{1000}, L_{1250} \\
L_{1500}, L_{\infty}\end{array}$ & $\begin{array}{l}L_{K}> \\
L_{K}= \\
L_{K}<\end{array}$ & $\begin{array}{l}L_{0}, L_{100}, L_{250}, L_{500}, L_{750}, L_{1000}, L_{1250} \\
L_{1500}, L_{\infty}\end{array}$ & $\begin{array}{l}L_{K}> \\
L_{K}= \\
L_{K}<\end{array}$ & $\begin{array}{l}L_{0}, L_{100}, L_{250}, L_{500}, L_{750}, L_{1000}, L_{1250}, \\
L_{1500}, L_{\infty}\end{array}$ \\
\hline$D=10$ & $\begin{array}{l}L_{K}> \\
L_{K}= \\
L_{K}<\end{array}$ & $\begin{array}{l}L_{0}, L_{100}, L_{250}, L_{500}, L_{750}, L_{1000}, L_{1250} \\
L_{1500}, L_{\infty}\end{array}$ & $\begin{array}{l}L_{K}> \\
L_{K}= \\
L_{K}<\end{array}$ & $\begin{array}{l}L_{0}, L_{100}, L_{250}, L_{500}, L_{750}, L_{1000}, L_{1250} \\
L_{1500}, L_{\infty}\end{array}$ & $\begin{array}{l}L_{K}> \\
L_{K}= \\
L_{K}<\end{array}$ & $\begin{array}{l}L_{0}, L_{100}, L_{250}, L_{500}, L_{750}, L_{1000}, L_{1250}, \\
L_{1500}, L_{\infty}\end{array}$ \\
\hline$D=30$ & $\begin{array}{l}L_{K}> \\
L_{K}= \\
\\
L_{K}<\end{array}$ & $\begin{array}{l}L_{0}, L_{100}, L_{250}, L_{500}, L_{750}, L_{1000}, L_{1250} \\
L_{1500}, L_{\infty}\end{array}$ & $\begin{array}{l}L_{K}> \\
L_{K}= \\
\\
L_{K}<\end{array}$ & $\begin{array}{l}L_{0}, L_{100}, L_{250}, L_{500}, L_{750}, L_{1000}, L_{1250} \\
L_{1500}, L_{\infty}\end{array}$ & $\begin{array}{l}L_{K}> \\
L_{K}= \\
\\
L_{K}<\end{array}$ & $\begin{array}{l}L_{0}, L_{100}, L_{250}, L_{500}, L_{750}, L_{1000}, L_{1250}, \\
L_{1500}, L_{\infty}\end{array}$ \\
\hline$D=50$ & $\begin{array}{l}L_{K}> \\
L_{K}= \\
\\
L_{K}<\end{array}$ & $\begin{array}{l}L_{0}, L_{100}, L_{250}, L_{500}, L_{750}, L_{1000}, L_{1250} \\
L_{1500}, L_{\infty}\end{array}$ & $\begin{array}{l}L_{K}> \\
L_{K}= \\
\\
L_{K}<\end{array}$ & $\begin{array}{l}L_{0}, L_{100}, L_{250}, L_{500}, L_{750}, L_{1000}, L_{1250} \\
L_{1500}, L_{\infty}\end{array}$ & $\begin{array}{l}L_{K}> \\
L_{K}= \\
\\
L_{K}<\end{array}$ & $\begin{array}{l}L_{0}, L_{100}, L_{250}, L_{500}, L_{750}, L_{1000}, L_{1250}, \\
L_{1500}, L_{\infty}\end{array}$ \\
\hline
\end{tabular}

Table 25

$f_{1}$, horizontal approach, $10^{-6}$, large dimension, NHST

\begin{tabular}{|c|c|c|c|c|c|c|}
\hline & & $\mathrm{SN}=24$ & & $\mathrm{SN}=\mathbf{5 0}$ & & $\mathrm{SN}=100$ \\
\hline$D=100$ & $\begin{array}{l}L_{K}= \\
L_{K}<\end{array}$ & $\begin{array}{l}L_{2000} *, L_{3000} *, L_{4000} *, L_{5000} *, L_{6000} * \\
L_{7000} *, L_{8000} *, L_{9000}, L_{10000} *, L_{11000} * \\
L_{12000} *, L_{13000} *, L_{14000} *, L_{15000} *, L_{\infty} *\end{array}$ & $\begin{array}{l}L_{K}= \\
L_{K}<\end{array}$ & $\begin{array}{l}L_{2000}, L_{3000}, L_{4000}, L_{5000}, L_{6000}, L_{7000} \\
L_{9000}, L_{10000}, L_{11000}, L_{12000}, L_{13000} \\
L_{14000}, L_{15000}, L_{\infty}\end{array}$ & $\begin{array}{l}L_{K}> \\
L_{K}= \\
L_{K}<\end{array}$ & $\begin{array}{l}L_{0} *, L_{1000} *, L_{2000}, L_{7000}, L_{13000}, L_{15000}, \\
L_{\infty} \\
L_{5000} \\
L_{3000}, L_{4000}, L_{6000}, L_{8000}, L_{9000}, L_{10000}, \\
L_{11000}, L_{12000}, L_{14000}\end{array}$ \\
\hline$D=200$ & $\begin{array}{l}L_{K}= \\
L_{K}<\end{array}$ & $\begin{array}{l}L_{3000}, L_{4000} *, \quad L_{5000} *, L_{6000} *, L_{7000} * \\
L_{8000}, \quad L_{9000}, \quad L_{10000}, \quad L_{11000} * \\
L_{12000} *, L_{13000}, L_{14000} *, L_{15000} *, L_{\infty} *\end{array}$ & $\begin{array}{l}L_{K}= \\
L_{K}<\end{array}$ & $\begin{array}{l}L_{0} *, L_{1000} *, L_{2000} *, L_{3000}, L_{4000}, L_{6000}, \\
L_{7000}, L_{8000}, L_{9000}, L_{10000}, L_{11000}, \\
L_{12000}, L_{13000}, L_{14000}, L_{15000}, L_{\infty} \\
L_{5000}\end{array}$ & $\begin{array}{l}L_{K}= \\
L_{K}<\end{array}$ & $\begin{array}{l}L_{0} *, L_{1000} *, L_{2000} *, L_{3000} *, L_{12000}, \\
L_{14000}, L_{15000}, L_{\infty} \\
L_{10000} \\
L_{4000}, L_{5000}, L_{6000}, L_{7000}, L_{8000}, L_{9000}, \\
L_{11000}, L_{13000}\end{array}$ \\
\hline$D=300$ & $\begin{array}{l}L_{K}= \\
L_{K}<\end{array}$ & $\begin{array}{l}L_{4000}, \quad L_{5000} *, \quad L_{6000} *, L_{7000} *, L_{8000} * \\
L_{9000 *,} \quad L_{10000 *} \quad L_{11000} *, \quad L_{12000} * \\
L_{13000} *, L_{14000} *, L_{15000} *, L_{\infty} *\end{array}$ & $\begin{array}{l}L_{K}> \\
L_{K}= \\
L_{K}<\end{array}$ & $\begin{array}{l}L_{0} *, \quad L_{1000} *, \quad L_{2000} *, \quad L_{3000} *, \quad L_{4000}, \\
L_{5000}, L_{8000}, L_{10000}, L_{11000}, L_{12000}, \\
L_{13000}, L_{15000}, L_{\infty} \\
L_{6000}, L_{7000}, L_{9000}, L_{14000}\end{array}$ & $\begin{array}{l}L_{K}> \\
L_{K}= \\
L_{K}<\end{array}$ & $\begin{array}{l}L_{0} *, \quad L_{1000} *, L_{2000} *, L_{3000} *, L_{4000}, \\
L_{5000}, L_{6000}, L_{7000}, L_{8000}, L_{9000}, L_{11000}, \\
L_{14000}, L_{\infty} \\
L_{15000} \\
L_{10000}, L_{12000}, L_{13000}\end{array}$ \\
\hline
\end{tabular}

Table 26

$f_{2}$, horizontal approach, $10^{-6}$, large dimension, NHST

\begin{tabular}{|c|c|c|c|c|c|c|}
\hline & & $\mathrm{SN}=24$ & & $\mathrm{SN}=50$ & & $\mathrm{SN}=100$ \\
\hline \multirow{3}{*}{$\mathrm{D}=\mathbf{1 0 0}$} & $L_{K}>$ & $L_{0} *, L_{4000}, L_{7000}, L_{8000}, L_{13000}, L_{\infty}$ & $L_{K}>$ & $\begin{array}{l}L_{0} *, L_{1000}, L_{3000}, L_{4000}, L_{5000}, L_{6000}, \\
L_{8000}, L_{12000}, L_{\infty}\end{array}$ & $L_{K}>$ & $\begin{array}{l}L_{0} *, L_{1000}, L_{2000}, L_{3000}, L_{4000}, L_{6000}, \\
L_{7000}, L_{9000} L_{10000}, L_{11000}, L_{12000}, \\
L_{13000}, L_{14000}, L_{15000}, L_{\infty}\end{array}$ \\
\hline & $L_{K}=$ & & $L_{K}=$ & & $L_{K}=$ & $L_{5000}$ \\
\hline & $L_{K}<$ & $\begin{array}{l}L_{1000}, L_{2000}, L_{3000}, L_{5000}, L_{6000}, L_{9000}, \\
L_{10000}, L_{11000}, L_{12000}, L_{14000}, L_{15000}\end{array}$ & $L_{K}<$ & $\begin{array}{l}L_{2000}, L_{7000}, L_{9000}, L_{10000}, L_{11000}, \\
L_{13000}, L_{14000}, L_{15000}\end{array}$ & $L_{K}<$ & $L_{8000}$ \\
\hline \multirow{2}{*}{$\mathrm{D}=\mathbf{2 0 0}$} & $L_{K}>$ & $\begin{array}{l}L_{0} *, L_{1000}, L_{2000}, L_{3000}, L_{4000}, L_{5000}, \\
L_{6000}, L_{7000}, L_{8000}, L_{9000}, L_{10000}, L_{11000}, \\
L_{14000}, L_{15000}, L_{\infty}\end{array}$ & $L_{K}>$ & $L_{0} *, L_{1000}$ & $L_{K}>$ & $\begin{array}{l}L_{0} *, L_{1000}, L_{3000}, L_{4000}, L_{5000}, L_{6000}, \\
L_{7000}, L_{8000}, L_{9000}, L_{11000}, L_{12000}, \\
L_{13000}, L_{14000}, L_{15000}, L_{\infty} \\
L_{10000}\end{array}$ \\
\hline & $L_{K}<$ & $L_{12000}, L_{13000}$ & $L_{K}<$ & $\begin{array}{l}L_{2000}, L_{3000}, L_{4000}, L_{6000}, L_{7000}, L_{8000}, \\
L_{9000}, L_{10000}, L_{11000}, L_{12000}, L_{13000}, \\
L_{14000}, L_{15000}, L_{\infty}\end{array}$ & $L_{K}<$ & $L_{2000}$ \\
\hline \multirow{3}{*}{$\mathrm{D}=\mathbf{3 0 0}$} & $L_{K}>$ & $\begin{array}{l}L_{0} *, L_{1000}, L_{2000}, L_{3000}, L_{4000}, L_{8000}, \\
L_{9000}\end{array}$ & $L_{K}>$ & $\begin{array}{l}L_{0} *, L_{1000}, L_{2000}, L_{3000}, L_{8000}, L_{9000}, \\
L_{10000}, L_{11000}, L_{14000}, L_{15000}, L_{\infty}\end{array}$ & $L_{K}>$ & \multirow{3}{*}{$\begin{array}{l}L_{0 *}, L_{1000}, L_{2000}, L_{3000}, L_{4000}, L_{5000}, \\
L_{6000}, L_{7000}, L_{8000}, L_{9000}, L_{10000}, L_{11000}, \\
L_{12000}, L_{13000}, L_{14000}, L_{\infty} \\
L_{15000}\end{array}$} \\
\hline & $L_{K}=$ & & $L_{K}=$ & & $L_{K}=$ & \\
\hline & $L_{K}<$ & $\begin{array}{l}L_{5000}, L_{6000}, L_{7000}, L_{10000}, L_{11000}, \\
L_{12000}, L_{13000}, L_{14000}, L_{15000}, L_{\infty}\end{array}$ & $L_{K}<$ & $L_{4000}, L_{5000}, L_{6000}, L_{7000}, L_{12000}, L_{13000}$ & $L_{K}<$ & \\
\hline
\end{tabular}


Table 27

$f_{3}$, horizontal approach, $10^{-6}$, large dimension, NHST

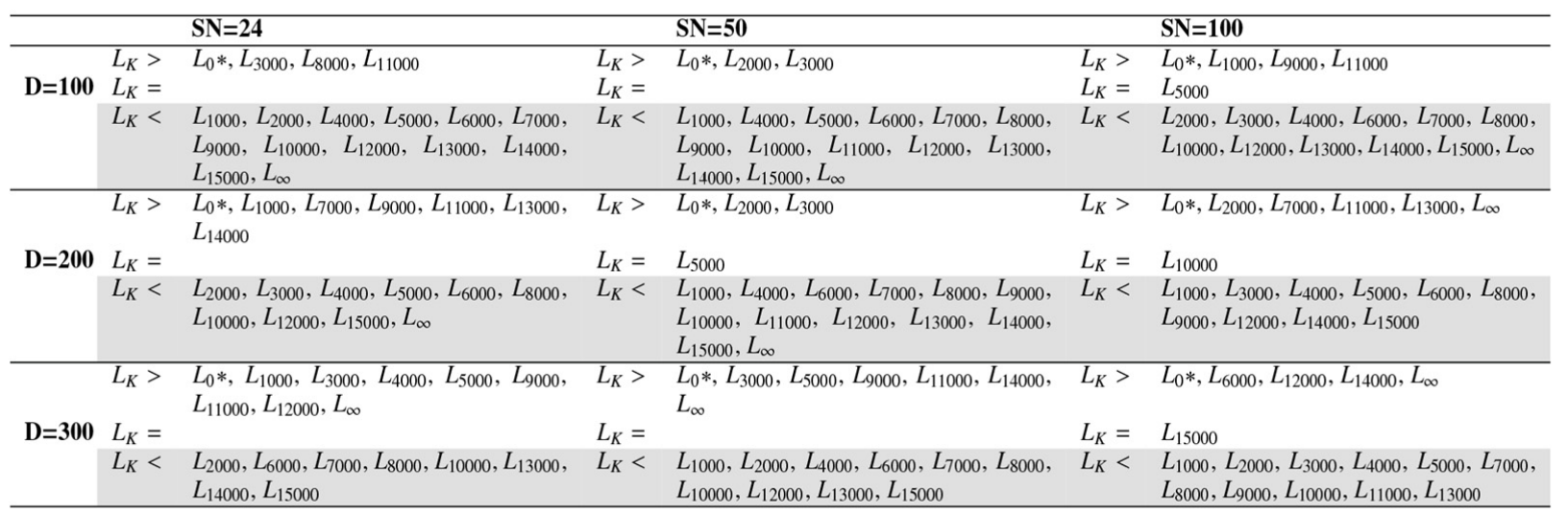

Table 28

$f_{4}$, horizontal approach, $10^{-6}$, large dimension, NHST

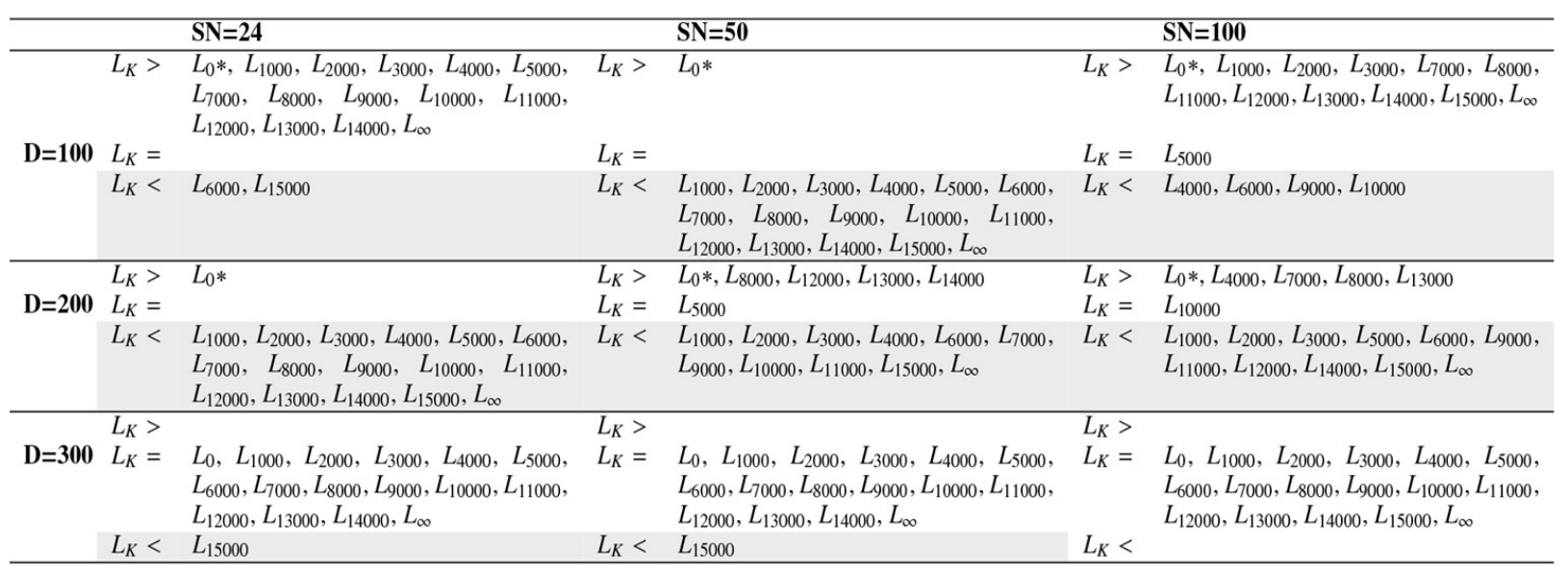

Table 29

$f_{5}$, horizontal approach, $10^{-6}$, large dimension, NHST

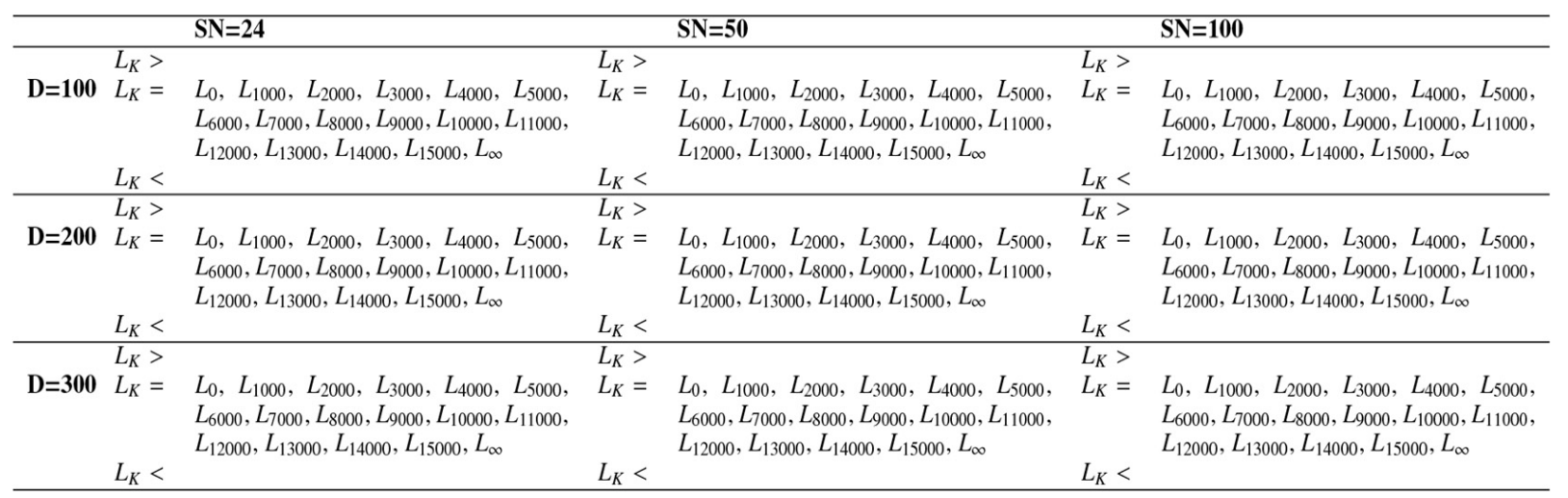


experiment showed that there are other 'limit' values that perform better than $L_{k}$, for certain problems $\left(f_{1}\right)$ even significantly. In almost all $D$ and $S N$ settings and problems, at least one better performing 'limit' value was found (the only exceptions are $f_{1}, S N=50$, and $D=$ 200 and $f_{2}, S N=100$, and $D=300$ ). For $f_{5}$, none of the 'limit' values reached optimal solution, since all settings performed equally. By comparing Tables 25-29 with Tables 15-19, it can be observed that with higher dimensions $L_{k}$ setting becomes less appropriate.

\subsubsection{Discussion}

The analysis with NHST supported our concerns about setting a fixed 'limit' value regarding the suggested formula $L_{k}=\mathrm{n}_{\mathrm{e}}{ }^{*} D=(S N / 2){ }^{*} D$. When a smaller number of fitness evaluations (e.g., 100,000) were available, $L_{k}$ was the appropriate choice only for small dimensions ( $D=2$, rarely for $D=5$ or $D=10)$ amongst all the five presented problems. When dimension got bigger, more appropriate alternatives could be chosen. On the other hand, when sufficiently large enough numbers of fitness evaluations were available (e.g., $250,000), L_{k}$ was a significantly better choice than the presented fixed 'limit' values for all the presented problems, dimensions, and values of population size. This does not necessarily mean that a better value than $L_{k}$ does not exist but it was not defined in our set of fixed 'limit' values.

When it was of interest in finding a (sub-)optimal solution (i.e., $10^{-6}$ ) and a larger number of fitness evaluations were available (i.e., 1,000,000), $L_{k}$ has better alternatives for the all presented problems, dimensions, and values of population size. The only time $L_{k}$ seemed to be like an appropriate choice was for problem $f_{1}$ (multi-modal, non-separable problem) when population size equaled 50 and for problem $f_{5}$ (uni-modal, non-separable problem) for which ABC did not reach the given (sub-)optimal solution over $1,000,000$ fitness evaluations regardless of the 'limit' value. When the value of this (sub-)optimal solution was even more precise (i.e., $10^{-12}$ ) there were better alternatives than $L_{k}$ even for problem $f_{1}$. In summary, it was shown that even within this small benchmark suite used in our study setting 'limit' is very problem dependent (e.g., see Tables 5-9 for results on $f_{1}-f_{5}$ ).

In many cases, better settings existed (even significantly better) than setting 'limit' according to the suggested formula. The results also heavily depended on the number of available fitness evaluations, indi- cating that $\mathrm{ABC}$ convergence with $L_{k}$ is not amongst the fastest. The results from the horizontal approach further supported this claim.

\subsection{Chess Rating System for Evolutionary Algorithms (CRS4EAs)}

The Chess Rating System for Evolutionary Algorithms (CRS4EAs) [48] is a novel method for the comparing and ranking of evolutionary algorithms. In this method, each participating algorithm plays the role of a chess player. The comparison between two players is treated as one game that can have only one out of three outcomes: win, lose, or draw. Two algorithms play a draw whenever the difference in their solutions is smaller than predefined $\varepsilon$. Otherwise, the algorithm with the solution closer to the optimum of an optimisation problem wins and the other loses. A pairwise comparison between the solutions of all participating algorithms on all optimisation problems over all independent runs is treated as one tournament. After the tournament has been conducted, the rating $R$, rating deviation $R D$, and rating interval $R I$ for each of the players are calculated regarding the formula from the Glicko-2 rating system [16], [17]. Rating is an absolute power of a player that is supported by rating deviation. The higher the rating deviation, the less reliable the player's rating. Rating interval is formed from rating and rating deviation. It can be said with $95 \%$ probability that a player's rating $R$ belongs to an interval $[R-2 R D, R+2 R D]$. Regarding these rating intervals, the algorithms can then be compared and if their intervals do not overlap, the algorithms are considered significantly different. The result of one such tournament is a leaderboard from which all these data can be read and interpreted. When players enter a tournament their rating power equals 1500 , and their rating deviation equals 350 , which is the maximum available rating deviation value. The more games the algorithms play, the smaller become the rating deviation values, and the minimum value usually used in CRS4EAs comparisons equals 50.

In this analysis, players were presented as $\mathrm{ABC}$ algorithms with different 'limit' value settings. A tournament was executed for each combination of $S N$ and $D$ values for each optimisation problem separately to allow fair comparison with NHST's Wilcoxon's test. The results of both analyses (NHST's and CRS4EAs') were very similar, however, there were some differ- 
ences. Even though, both the Wilcoxon's test and CRS4EAs compared all runs pairwisely, the results of the Wilcoxon's test were more relative and the results of CRS4EAs' more absolute. The Wilcoxon's test took into consideration only wins and losses against $L_{k}$, which were reflected in the $p$ value. CRS4EAs, on the other hand, conducted a tournament between 10 players $\left(L_{k}, L_{0}, L_{100}, L_{250}, L_{500}, L_{750}, L_{1000}, L_{1250}, L_{1500}, L_{\infty}\right)$ where runs were pairwisely compared. In regard to these wins, losses, and draws, a rating was calculated and not only were the games against $L_{k}$ taken into consideration but games against all opponents. This is the main reason behind the differences between the results of both methods. However, to point out once again: in both approaches, the results were compared as $1 \times k$ comparison as CRS4EAs being appropriate for both types of comparisons $-1 \times k$ and $k \times k$. There was also a difference in effort put into executing both methods. In CRS4EAs when the ratings of pairwise comparison were obtained, there was no need for further calculations and testing, whilst when $p$ values are calculated with a statistical test, a post-hoc test, such as the Holm test, is always necessary due to the repetitive comparisons of $L_{k}$ with other settings.

The experiment was again divided into 4 parts for CRS4EAs analysis. Each part of the experiment took a different approach just as the ones shown in Table 3. For a more straightforward comparison, the reports of rating deviations and rating intervals were omitted in the tables with results, even though they were calculated and used in detecting significant differences. The $\varepsilon$ for determining the draw was set to $10^{-20}$ as results were compared up to 20 decimals places in the Wilcoxon's test as well. A less precise $\varepsilon$ would affect the detected differences and there would be greater differences in NHST and CRS4EAs analyses. The minimum rating deviation value was set at 50 and the maximum rating deviation value at 350 . Glicko-2 also calculates some other measurements we omitted during this analysis, as they were unimportant in this analysis. The other CRS4EAs' parameters used in formulae for calculating rating and rating deviation were determined regarding the Glicko-2 rating system. Readers can find more on this topic and definitions of these parameters in [26].

\subsubsection{Experiment 1: Vertical Approach with MaxFEs $=100,000$}

Tables 30(a) - 30(e) showed the ratings obtained for every setting of $S N$ and $D$ for all 5 minimisation prob- lems. All the players reached the minimum rating deviation value of 50 rating points. The best player of each setting (shown in one row) is marked in light grey background colour. For example, from Table $30(S N=$ 24, $D=10$ ), it can be observed that the highest rating of 1768 points was obtained using $L_{250}$ followed by $L_{500}$ (1693 points), $L_{1000}$ (1631 points), $L_{750}$ (1628 points), $L_{1500}$ (1603 points), $L_{1250}$ (1597 points), $L_{\infty}$ (1588 points), $L_{k}$ (1394 points), $L_{100}$ (1116 points), and $L_{0}$ (982 points). The difference in rating between the winner $L_{250}$ (1768 points) and $L_{k}$ (1394 points) was more than 200 points $(4 R D)$ and hence statistically significant. Overall, these tables show that $L_{k}$ was not always the more appropriate value for 'limit' - especially for $f_{5}$. However, observing the ratings and when calculating the rating intervals as $[R-100, R+100]$ where 100 is $2 * R D_{\text {min }}=2 * 50$, the differences were rarely significant.

Tables 31-35 show more clearly the differences found between $L_{k}$ and the other 9 fixed 'limit' values on all 5 optimisation problems. Whenever the difference was significant, the star symbol $\left(^{*}\right)$ has been placed after

\section{Table 30}

Vertical approach, MaxFEs $=100,000$

\begin{tabular}{lcccccccccc}
\hline & $L_{K}$ & $L_{0}$ & $L_{100}$ & $L_{250}$ & $L_{500}$ & $L_{750}$ & $L_{1000}$ & $L_{1250}$ & $L_{1500}$ & $L_{\infty}$ \\
\hline $\mathrm{SN}=24 \mathrm{D}=2$ & 1562 & 1029 & 1562 & 1562 & 1557 & 1562 & 1557 & 1546 & 1540 & 1525 \\
$\mathrm{SN}=24 \mathrm{D}=5$ & 1478 & 981 & 1578 & 1578 & 1573 & 1578 & 1553 & 1563 & 1558 & 1559 \\
$\mathrm{SN}=24 \mathrm{D}=10$ & 1394 & 982 & 1116 & 1768 & 1693 & 1628 & 1631 & 1597 & 1603 & 1588 \\
$\mathrm{SN}=24 \mathrm{D}=30$ & 1518 & 1012 & 1072 & 1265 & 1650 & 1717 & 1705 & 1690 & 1680 & 1690 \\
$\mathrm{SN}=24 \mathrm{D}=50$ & 1619 & 1033 & 1074 & 1247 & 1508 & 1693 & 1726 & 1713 & 1694 & 1693 \\
$\mathrm{SN}=50 \mathrm{D}=2$ & 1558 & 1018 & 1558 & 1558 & 1558 & 1558 & 1553 & 1542 & 1558 & 1541 \\
$\mathrm{SN}=50 \mathrm{D}=5$ & 1571 & 981 & 1571 & 1571 & 1566 & 1545 & 1561 & 1561 & 1540 & 1535 \\
$\mathrm{SN}=50 \mathrm{D}=10$ & 1745 & 981 & 1122 & 1745 & 1697 & 1614 & 1582 & 1591 & 1547 & 1622 \\
$\mathrm{SN}=50 \mathrm{D}=30$ & 1682 & 1021 & 1074 & 1301 & 1635 & 1682 & 1691 & 1696 & 1675 & 1725 \\
$\mathrm{SN}=50 \mathrm{D}=50$ & 1688 & 1073 & 1094 & 1249 & 1577 & 1705 & 1684 & 1688 & 1710 & 1719 \\
$\mathrm{SN}=100 \mathrm{D}=2$ & 1565 & 1051 & 1565 & 1565 & 1565 & 1565 & 1554 & 1543 & 1549 & 1543 \\
$\mathrm{SN}=100 \mathrm{D}=5$ & 1569 & 981 & 1575 & 1569 & 1575 & 1559 & 1564 & 1543 & 1559 & 1575 \\
$\mathrm{SN}=100 \mathrm{D}=10$ & 1637 & 981 & 1113 & 1711 & 1637 & 1579 & 1643 & 1611 & 1601 & 1624 \\
$\mathrm{SN}=100 \mathrm{D}=30$ & 1705 & 1021 & 1083 & 1278 & 1666 & 1710 & 1679 & 1721 & 1705 & 1636 \\
$\mathrm{SN}=100 \mathrm{D}=50$ & 1650 & 1060 & 1060 & 1247 & 1530 & 1645 & 1711 & 1718 & 1660 & 1717 \\
\hline
\end{tabular}
(a) Vertical approach, $f_{1}$, MaxFEs $=100,000$

\begin{tabular}{lccccccccccc}
\hline & $L_{K}$ & $L_{0}$ & $L_{100}$ & $L_{250}$ & $L_{500}$ & $L_{750}$ & $L_{1000}$ & $L_{1250}$ & $L_{1500}$ & $L_{\infty}$ \\
\hline $\mathrm{SN}=24 \mathrm{D}=2$ & 1827981 & 1795 & 1648 & 1556 & 1495 & 1455 & 1447 & 1451 & 1344 \\
$\mathrm{SN}=24 \mathrm{D}=5$ & 1689 & 981 & 1654 & 1689 & 1599 & 1601 & 1620 & 1422 & 1362 & 1383 \\
$\mathrm{SN}=24 \mathrm{D}=10$ & 1566 & 981 & 1576 & 1538 & 1579 & 1574 & 1555 & 1542 & 1521 & 1569 \\
$\mathrm{SN}=24 \mathrm{D}=30$ & 1574 & 981 & 1577 & 1588 & 1495 & 1583 & 1545 & 1547 & 1582 & 1528 \\
$\mathrm{SN}=24 \mathrm{D}=50$ & 1525 & 981 & 1611 & 1542 & 1537 & 1570 & 1527 & 1540 & 1567 & 1600 \\
$\mathrm{SN}=50 \mathrm{D}=2$ & 1779981 & 1767 & 1627 & 1596 & 1532 & 1456 & 1475 & 1429 & 1358 \\
$\mathrm{SN}=50 \mathrm{D}=5$ & 1725981 & 1648 & 1640 & 1574 & 1603 & 1600 & 1420 & 1370 & 1441 \\
$\mathrm{SN}=50 \mathrm{D}=10$ & 1596981 & 1583 & 1596 & 1573 & 1542 & 1566 & 1543 & 1544 & 1573 \\
$\mathrm{SN}=50 \mathrm{D}=30$ & 1544 & 981 & 1584 & 1561 & 1562 & 1544 & 1542 & 1570 & 1618 & 1538 \\
$\mathrm{SN}=50 \mathrm{D}=50$ & 1600981 & 1560 & 1591 & 1578 & 1555 & 1578 & 1516 & 1600 & 1543 \\
$\mathrm{SN}=100 \mathrm{D}=2$ & 1793981 & 1793 & 1677 & 1610 & 1497 & 1553 & 1511 & 1529 & 1349 \\
$\mathrm{SN}=100 \mathrm{D}=5$ & 1741 & 981 & 1679 & 1741 & 1602 & 1616 & 1525 & 1492 & 1462 & 1401 \\
$\mathrm{SN}=100 \mathrm{D}=10$ & 1542 & 981 & 1617 & 1529 & 1542 & 1560 & 1571 & 1540 & 1578 & 1583 \\
$\mathrm{SN}=100 \mathrm{D}=30$ & 1573 & 981 & 1574 & 1556 & 1608 & 1534 & 1590 & 1574 & 1573 & 1512 \\
$\mathrm{SN}=100 \mathrm{D}=50$ & 1544 & 981 & 1544 & 1539 & 1555 & 1570 & 1515 & 1599 & 1552 & 1600 \\
\hline
\end{tabular}
(b) Vertical approach, $f_{2}$, MaxFEs $=100,000$ 
\begin{tabular}{lllllllllll}
\hline & $L_{K}$ & $L_{0}$ & $L_{100}$ & $L_{250}$ & $L_{500}$ & $L_{750}$ & $L_{1000}$ & $L_{1250}$ & $L_{1500}$ & $L_{\infty}$ \\
\hline $\mathrm{SN}=240$ &
\end{tabular} \begin{tabular}{llllllllllll}
\hline $\mathrm{SN}=24 \mathrm{D}=2$ & 1557991 & 1557 & 1557 & 1557 & 1557 & 1557 & 1557 & 1557 & 1557
\end{tabular}

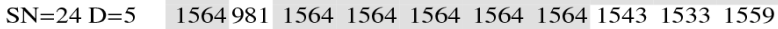
$\mathrm{SN}=24 \mathrm{D}=10 \quad 15809811554 \quad 1570 \quad 1554 \quad 15541544 \quad 1570 \quad 15441549$ $\mathrm{SN}=24 \mathrm{D}=30 \quad 15179811514 \quad 1604 \quad 1569 \quad 1578 \quad 1546 \quad 1616 \quad 1547 \quad 1528$

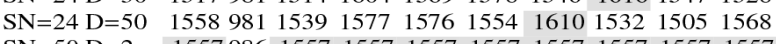

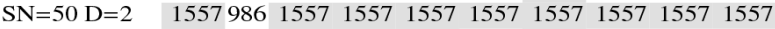

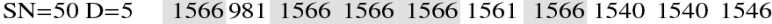
$\begin{array}{llllllllllllll}\mathrm{SN}=50 & \mathrm{D}=10 & 1560 & 981 & 1596 & 1560 & 1549 & 1560 & 1560 & 1570 & 1560 & 1565\end{array}$

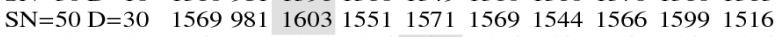
$\begin{array}{llllllllllll}\mathrm{SN}=50 \mathrm{D}=50 & 1568 & 981 & 1545 & 1536 & 1608 & 1536 & 1561 & 1568 & 1569 & 1596\end{array}$

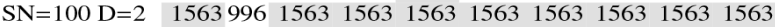

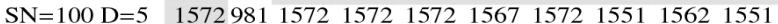

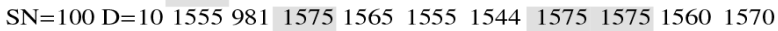

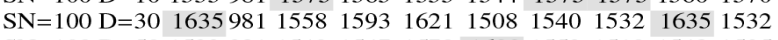

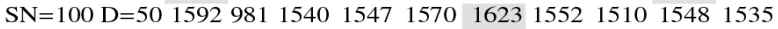

(c) Vertical approach, $f_{3}$, MaxFEs $=100,000$

\begin{tabular}{lllllllllll}
\hline$L_{K}$ & $L_{0}$ & $L_{100}$ & $L_{250}$ & $L_{500}$ & $L_{750}$ & $L_{1000}$ & $L_{1250}$ & $L_{1500}$ & $L_{\infty}$
\end{tabular}

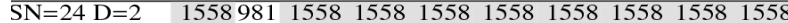
$\begin{array}{lllllllllll}\mathrm{SN}=24 & \mathrm{D}=5 & 1558981 & 1558 & 1558 & 1558 & 1558 & 1558 & 1558 & 1558 & 1558\end{array}$

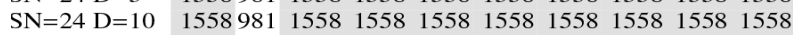

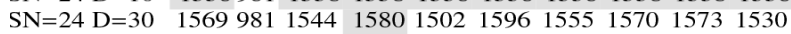

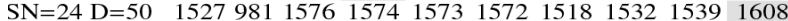
$\begin{array}{llllllllllll}\mathrm{SN}=50 & \mathrm{D}=2 & 1558981 & 1558 & 1558 & 1558 & 1558 & 1558 & 1558 & 1558 & 1558\end{array}$ $\begin{array}{lllllllllll}\mathrm{SN}=50 & \mathrm{D}=5 & 1558981 & 1558 & 1558 & 1558 & 1558 & 1558 & 1558 & 1558 & 1558\end{array}$ $\begin{array}{llllllllllll}\mathrm{SN}=50 & \mathrm{D}=10 & 1565981 & 1565 & 1565 & 1565 & 1565 & 1565 & 1565 & 1565 & 1565\end{array}$

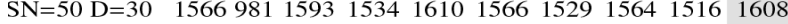
$\begin{array}{llllllllllll}\mathrm{SN}=50 \mathrm{D}=50 & 1595 & 981 & 1553 & 1538 & 1549 & 1547 & 1599 & 1595 & 1586 & 1553\end{array}$

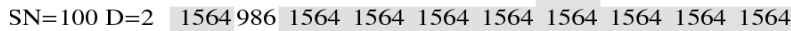
$\begin{array}{llllllllllll}\mathrm{SN}=100 & \mathrm{D}=5 & 1565981 & 1565 & 1565 & 1565 & 1565 & 1565 & 1565 & 1565 & 1565\end{array}$

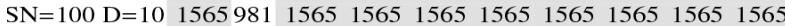
$\mathrm{SN}=100 \mathrm{D}=30 \quad 1570981 \quad 1543 \quad 1561 \quad 1574 \quad 1587 \quad 1575 \quad 1566 \quad 1570 \quad 1543$

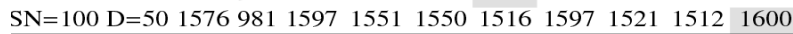
(d) Vertical approach, $f_{4}$, MaxFEs $=100,000$

\begin{tabular}{lcccccccccc}
\hline & $L_{K}$ & $L_{0}$ & $L_{100}$ & $L_{250}$ & $L_{500}$ & $L_{750}$ & $L_{1000}$ & $L_{1250}$ & $L_{1500}$ & $L_{\infty}$ \\
\hline $\mathrm{SN}=24 \mathrm{D}=2$ & 1563 & 1502 & 1512 & 1521 & 1509 & 1510 & 1471 & 1503 & 1532 & 1375 \\
$\mathrm{SN}=24 \mathrm{D}=5$ & 1261 & 982 & 1371 & 1498 & 1598 & 1612 & 1637 & 1650 & 1672 & 1719 \\
$\mathrm{SN}=24 \mathrm{D}=10$ & 1423 & 981 & 1359 & 1538 & 1518 & 1702 & 1612 & 1620 & 1619 & 1628 \\
$\mathrm{SN}=24 \mathrm{D}=30$ & 1558 & 981 & 1521 & 1602 & 1593 & 1570 & 1583 & 1528 & 1551 & 1514 \\
$\mathrm{SN}=24 \mathrm{D}=50$ & 1536 & 981 & 1570 & 1567 & 1574 & 1537 & 1553 & 1521 & 1551 & 1611 \\
$\mathrm{SN}=50 \mathrm{D}=2$ & 1509 & 1505 & 1495 & 1537 & 1509 & 1516 & 1409 & 1525 & 1490 & 1505 \\
$\mathrm{SN}=50 \mathrm{D}=5$ & 1419 & 982 & 1322 & 1475 & 1589 & 1615 & 1673 & 1621 & 1644 & 1659 \\
$\mathrm{SN}=50 \mathrm{D}=10$ & 1551 & 981 & 1340 & 1551 & 1557 & 1582 & 1667 & 1641 & 1565 & 1616 \\
$\mathrm{SN}=50 \mathrm{D}=30$ & 1606 & 981 & 1571 & 1551 & 1597 & 1606 & 1513 & 1579 & 1532 & 1569 \\
$\mathrm{SN}=50 \mathrm{D}=50$ & 1532 & 981 & 1631 & 1484 & 1609 & 1606 & 1553 & 1532 & 1512 & 1591 \\
$\mathrm{SN}=100 \mathrm{D}=2$ & 1512 & 1535 & 1512 & 1514 & 1560 & 1534 & 1503 & 1482 & 1422 & 1439 \\
$\mathrm{SN}=100 \mathrm{D}=5$ & 1487 & 982 & 1327 & 1487 & 1580 & 1613 & 1613 & 1630 & 1636 & 1631 \\
$\mathrm{SN}=100 \mathrm{D}=10$ & 1616 & 981 & 1349 & 1534 & 1616 & 1614 & 1547 & 1644 & 1597 & 1618 \\
$\mathrm{SN}=100 \mathrm{D}=30$ & 1593 & 981 & 1593 & 1534 & 1588 & 1538 & 1614 & 1527 & 1593 & 1531 \\
$\mathrm{SN}=100 \mathrm{D}=50$ & 1487 & 981 & 1589 & 1597 & 1544 & 1560 & 1530 & 1576 & 1583 & 1553 \\
\hline
\end{tabular} (e) Vertical approach, $f_{5}$, MaxFEs $=100,000$ 'limit' value, and whenever the $L_{k}$ had better alternative(s) the background of table cell has been highlighted in light grey colour. Similar to the NHST approach, CRS4EAs also found significant difference only for $f_{1}$ and $f_{5}$. In particular, for $f_{1}: S N=24$ and $D=10$ where $L_{k}$ was significantly worse than $L_{250}, L_{500}, L_{750}, L_{1000}, L_{1250}$, $L_{1500}$. For $f_{5}: S N=24$ and $D=5$ where $L_{k}$ was significantly worse than $L_{250}, L_{500}, L_{750}, L_{1000}, L_{1250}, L_{1500}, L_{\infty} ; S N$ $=50$ and $D=5$ where $L_{k}$ was significantly worse than $L_{1000}, L_{1250}, L_{1500}, L_{\infty} ; S N=24$ and $D=10$ where $L_{k}$ was significantly worse than $L_{750}, L_{\infty}$. However, when comparing the detected significant differences between NHST and CRS4EAs (compare Tables 5-9 with Tables 31-35), CRS4EAs appears more conservative than NHST. Whilst the differences were presented for the same settings, in CRS4EAs these differences were hardly ever significant.

For all five problems, $L_{k}$ had almost always better alternatives (but not significant) when dimension $D$ was greater (10,30, or 50).

\subsubsection{Experiment 2: Vertical Approach with MaxFEs $=250,000$}

Tables 36(a)-36(e) show the ratings obtained for every setting of $S N$ and $D$ on all 5 minimisation problems. All players reached the minimum rating deviation value of 50 rating points. The best player of each setting (shown in one row) is marked in light grey background colour. These tables show that $L_{k}$ was almost always the more appropriate value for 'limit'. $f_{5}$ was the only problem for which better alternatives were found for some $S N$ and $D$ settings. Moreover, $L_{k}$ was just as in NHST analysis - the significantly better choice in most cases.

Table 31

$f_{1}$, vertical approach, MaxFEs $=100,000$, CRS4EAs

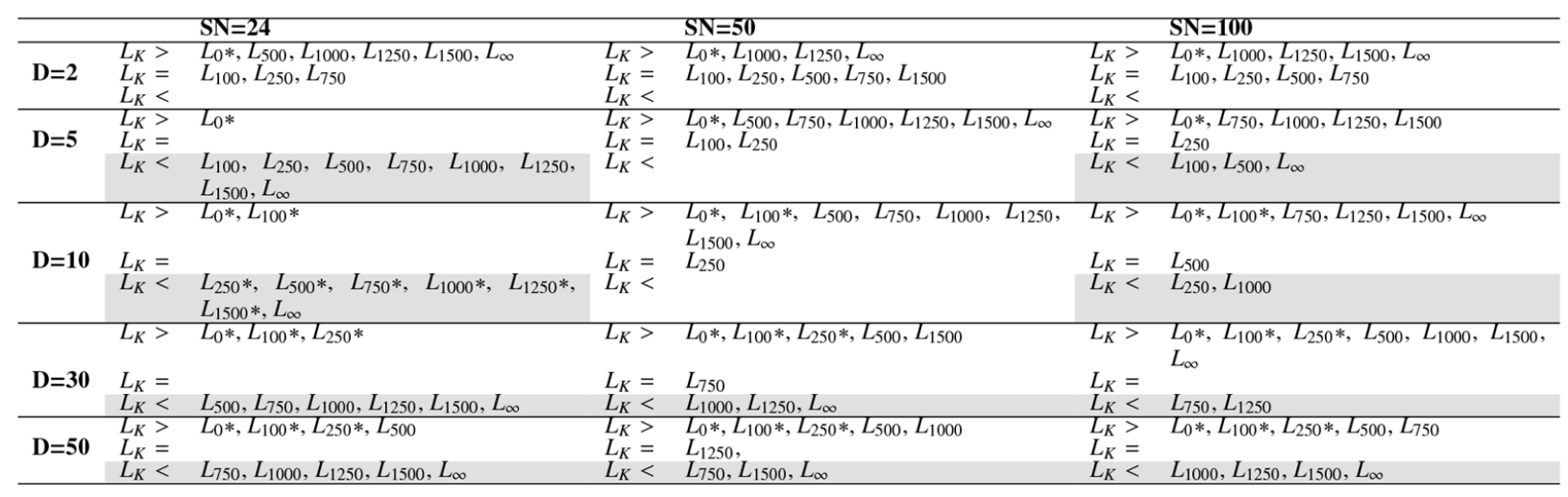


Table 32

$f_{2}$, vertical approach, MaxFEs $=100,000$, CRS4EAs

\begin{tabular}{|c|c|c|c|c|c|c|}
\hline & & $\mathrm{SN}=24$ & & $\mathrm{SN}=50$ & & $\mathrm{SN}=100$ \\
\hline $\mathrm{D}=2$ & $\begin{array}{l}L_{K}> \\
L_{K}= \\
L_{K}<\end{array}$ & $\begin{array}{l}L_{0} *, L_{100}, L_{250}, L_{500 *,} L_{750} *, L_{1000} * \\
L_{1250 *}, L_{1500 *}, L_{\infty} *\end{array}$ & $\begin{array}{l}L_{K}> \\
L_{K}= \\
L_{K}<\end{array}$ & $\begin{array}{l}L_{0} *, L_{100}, L_{250}, L_{500}, L_{750} *, L_{1000} * \text {, } \\
L_{1250}, L_{1500 *}, L_{\infty} *\end{array}$ & $\begin{array}{l}L_{K}> \\
L_{K}= \\
L_{K}<\end{array}$ & $\begin{array}{l}L_{0} *, L_{250}, L_{500}, L_{750} *, L_{1000} *, L_{1250 *}, \\
L_{1500 *}, L_{\infty} * \\
L_{100}\end{array}$ \\
\hline $\mathrm{D}=5$ & $\begin{array}{l}L_{K}> \\
L_{K}= \\
L_{K}<\end{array}$ & $\begin{array}{l}L_{0} *, L_{100}, L_{250}, L_{500}, L_{750}, L_{1000} \\
L_{1250 *}, L_{1500 *}, L_{\infty} *\end{array}$ & $\begin{array}{l}L_{K}> \\
L_{K}= \\
L_{K}<\end{array}$ & $\begin{array}{l}L_{0} *, L_{100}, L_{250}, L_{500}, L_{750}, L_{1000}, \\
L_{1250}, L_{1500 *}, L_{\infty} *\end{array}$ & $\begin{array}{l}L_{K}> \\
L_{K}= \\
L_{K}<\end{array}$ & $\begin{array}{l}L_{0} *, L_{100}, L_{500}, L_{750}, L_{1000} *, L_{1250} *, \\
L_{1500} * L_{\infty} * \\
L_{250}\end{array}$ \\
\hline $\mathrm{D}=\mathbf{1 0}$ & $\begin{array}{l}L_{K}= \\
L_{K}<\end{array}$ & $L_{0} *, L_{250}, L_{1000}, L_{1250}, L_{1500}$ & $\begin{array}{l}L_{K}= \\
L_{K}<\end{array}$ & $\begin{array}{l}L_{0} *, L_{100}, L_{500}, L_{750}, L_{1000}, L_{1250}, \\
L_{1500}, L_{\infty} \\
L_{250}\end{array}$ & $\begin{array}{l}L_{K}= \\
L_{K}<\end{array}$ & $\begin{array}{l}L_{0} *, L_{250}, L_{1250} \\
L_{500} \\
L_{100}, L_{750}, L_{1000}, L_{1500}, L_{\infty}\end{array}$ \\
\hline $\mathrm{D}=\mathbf{3 0}$ & $\begin{array}{l}L_{K}> \\
L_{K}= \\
L_{K}<\end{array}$ & $\begin{array}{l}L_{0} *, L_{500}, L_{1000}, L_{1250}, L_{\infty} \\
L_{100}, L_{250}, L_{500}, L_{750}, L_{1500}\end{array}$ & $\begin{array}{l}L_{K}> \\
L_{K}= \\
L_{K}<\end{array}$ & $\begin{array}{l}L_{0} *, L_{1000}, L_{\infty} \\
L_{750} \\
L_{100}, L_{250}, L_{500}, L_{1250}, L_{1500}\end{array}$ & $\begin{array}{l}L_{K}> \\
L_{K}= \\
L_{K}<\end{array}$ & $\begin{array}{l}L_{0} *, L_{250}, L_{750}, L_{\infty} \\
L_{1500} \\
L_{100}, L_{500}, L_{1000}, L_{1250}\end{array}$ \\
\hline $\mathrm{D}=\mathbf{5 0}$ & $\begin{array}{l}L_{K}> \\
L_{K}= \\
L_{K}<\end{array}$ & $\begin{array}{l}L_{0} * \\
L_{100}, L_{250}, L_{500}, L_{750}, L_{1000}, L_{1250}, \\
L_{1500}, L_{\infty}\end{array}$ & $\begin{array}{l}L_{K}> \\
L_{K}= \\
L_{K}<\end{array}$ & $\begin{array}{l}L_{0 *,} L_{100}, L_{250}, L_{500}, L_{750}, L_{1000}, L_{1500}, \\
L_{1250},\end{array}$ & $\begin{array}{l}L_{K}> \\
L_{K}= \\
L_{K}<\end{array}$ & $\begin{array}{l}L_{0} *, L_{250}, L_{1000} \\
L_{100} \\
L_{500}, L_{750}, L_{1250}, L_{1500}, L_{\infty}\end{array}$ \\
\hline
\end{tabular}

Table 33

$f_{3}$, vertical approach, MaxFEs $=100,000$, CRS4EAs

\begin{tabular}{|c|c|c|c|c|c|c|}
\hline & & $\mathrm{SN}=24$ & & $\mathrm{SN}=50$ & & $\mathrm{SN}=100$ \\
\hline $\mathrm{D}=\mathbf{2}$ & $\begin{array}{l}L_{K}> \\
L_{K}= \\
L_{K}<\end{array}$ & $\begin{array}{l}L_{0}^{*} \\
L_{100}, L_{250}, L_{500}, L_{750}, L_{1000}, L_{1250}, \\
L_{1500}, L_{\infty}\end{array}$ & $\begin{array}{l}L_{K}> \\
L_{K}= \\
L_{K}<\end{array}$ & $\begin{array}{l}L_{0} * \\
L_{100}, L_{250}, L_{500}, L_{750}, L_{1000}, L_{1250}, \\
L_{1500}, L_{\infty}\end{array}$ & $\begin{array}{l}L_{K}> \\
L_{K}= \\
L_{K}<\end{array}$ & $\begin{array}{l}L_{0} * \\
L_{100}, L_{250}, L_{500}, L_{750}, L_{1000}, L_{1250}, \\
L_{1500}, L_{\infty}\end{array}$ \\
\hline $\mathrm{D}=5$ & $\begin{array}{l}L_{K}> \\
L_{K}= \\
L_{K}<\end{array}$ & $\begin{array}{l}L_{0} *, L_{1250}, L_{1500}, L_{\infty} \\
L_{100}, L_{250}, L_{500}, L_{750}, L_{1000}\end{array}$ & $\begin{array}{l}L_{K}> \\
L_{K}= \\
L_{K}<\end{array}$ & $\begin{array}{l}L_{0} *, L_{750}, L_{1250}, L_{1500}, L_{\infty} \\
L_{100}, L_{250}, L_{500}, L_{1000}\end{array}$ & $\begin{array}{l}L_{K}> \\
L_{K}= \\
L_{K}<\end{array}$ & $\begin{array}{l}L_{0} *, L_{750}, L_{1250}, L_{1500}, L_{\infty} \\
L_{100}, L_{250}, L_{500}, L_{1000}\end{array}$ \\
\hline $\mathrm{D}=\mathbf{1 0}$ & $\begin{array}{l}L_{K}> \\
L_{K}= \\
L_{K}<\end{array}$ & $\begin{array}{l}L_{0} *, L_{100}, L_{250}, L_{500}, L_{750}, L_{1000}, L_{1250}, \\
L_{1500}, L_{\infty}\end{array}$ & $\begin{array}{l}L_{K}= \\
L_{K}<\end{array}$ & $\begin{array}{l}L_{250}, L_{750}, L_{1000}, L_{1500} \\
L_{100}, L_{1250}, L_{\infty}\end{array}$ & $\begin{array}{l}L_{K}= \\
L_{K}<\end{array}$ & $\begin{array}{l}L_{0} *, L_{750} \\
L_{500} \\
L_{100}, L_{250}, L_{1000}, L_{1250}, L_{1500}, L_{\infty}\end{array}$ \\
\hline $\mathrm{D}=\mathbf{3 0}$ & $\begin{array}{l}L_{K}= \\
L_{K}<\end{array}$ & $L_{250}, L_{500}, L_{750}, L_{1000}, L_{1250}, L_{1500}, L_{\infty}$ & $\begin{array}{l}L_{K}> \\
L_{K}= \\
L_{K}<\end{array}$ & $L_{0} *, L_{250}, L_{1000}, L_{1250}, L_{\infty}$ & $\begin{array}{l}L_{K}> \\
L_{K}= \\
L_{K}<\end{array}$ & $\begin{array}{l}L_{0} *, L_{100}, L_{250}, L_{500}, L_{750}, L_{1000}, L_{1250}, \\
L_{\infty} \\
L_{1500}\end{array}$ \\
\hline $\mathrm{D}=\mathbf{5 0}$ & $\begin{array}{l}L_{K}= \\
L_{K}<\end{array}$ & $L_{0} *, L_{100}, L_{750}, L_{1250}, L_{1500}$ & $\begin{array}{l}L_{K}> \\
L_{K}= \\
L_{K}<\end{array}$ & $L_{0} *, L_{100}, L_{250}, L_{750}, L_{1000}$ & $\begin{array}{l}L_{K}> \\
L_{K}= \\
L_{K}<\end{array}$ & $\begin{array}{l}L_{0} *, L_{100}, L_{250}, L_{500}, L_{1000}, L_{1250}, \\
L_{1500}, L_{\infty} \\
L_{750}\end{array}$ \\
\hline
\end{tabular}

Table 34

$f_{4}$, vertical approach, MaxFEs $=100,000$, CRS4EAs

\begin{tabular}{|c|c|c|c|c|c|c|}
\hline & & $\mathrm{SN}=24$ & & $\mathrm{SN}=50$ & & $\mathrm{SN}=100$ \\
\hline $\mathrm{D}=2$ & $\begin{array}{l}L_{K}> \\
L_{K}= \\
L_{K}<\end{array}$ & $\begin{array}{l}L_{0 *} \\
L_{100}, L_{250}, L_{500}, L_{750}, L_{1000}, L_{1250}, \\
L_{1500}, L_{\infty}\end{array}$ & $\begin{array}{l}L_{K}> \\
L_{K}= \\
L_{K}<\end{array}$ & $\begin{array}{l}L_{0} * \\
L_{100}, L_{250}, L_{500}, L_{750}, L_{1000}, L_{1250}, \\
L_{1500}, L_{\infty}\end{array}$ & $\begin{array}{l}L_{K}> \\
L_{K}= \\
L_{K}<\end{array}$ & $\begin{array}{l}L_{0 *} \\
L_{100}, L_{250}, L_{500}, L_{750}, L_{1000}, L_{1250}, \\
L_{1500}, L_{\infty}\end{array}$ \\
\hline $\mathrm{D}=5$ & $\begin{array}{l}L_{K}> \\
L_{K}= \\
L_{K}<\end{array}$ & $\begin{array}{l}L_{0 *} \\
L_{100}, L_{250}, L_{500}, L_{750}, L_{1000}, L_{1250}, \\
L_{1500}, L_{\infty}\end{array}$ & $\begin{array}{l}L_{K}> \\
L_{K}= \\
L_{K}<\end{array}$ & $\begin{array}{l}L_{0 *} \\
L_{100}, L_{250}, L_{500}, L_{750}, L_{1000}, L_{1250}, \\
L_{1500}, L_{\infty}\end{array}$ & $\begin{array}{l}L_{K}> \\
L_{K}= \\
L_{K}<\end{array}$ & $\begin{array}{l}L_{0 *}^{*} \\
L_{100}, L_{250}, L_{500}, L_{750}, L_{1000}, L_{1250}, \\
L_{1500}, L_{\infty}\end{array}$ \\
\hline $\mathrm{D}=10$ & $\begin{array}{l}L_{K}> \\
L_{K}= \\
L_{K}<\end{array}$ & $\begin{array}{l}L_{0} * \\
L_{100}, L_{250}, L_{500}, L_{750}, L_{1000}, L_{1250}, \\
L_{1500}, L_{\infty}\end{array}$ & $\begin{array}{l}L_{K}> \\
L_{K}= \\
L_{K}<\end{array}$ & $\begin{array}{l}L_{0} * \\
L_{100}, L_{250}, L_{500}, L_{750}, L_{1000}, L_{1250}, \\
L_{1500}, L_{\infty}\end{array}$ & $\begin{array}{l}L_{K}> \\
L_{K}= \\
L_{K}<\end{array}$ & $\begin{array}{l}L_{0 *} \\
L_{100}, L_{250}, L_{500}, L_{750}, L_{1000}, L_{1250}, \\
L_{1500}, L_{\infty}\end{array}$ \\
\hline $\mathrm{D}=\mathbf{3 0}$ & $\begin{array}{l}L_{K}> \\
L_{K}= \\
L_{K}<\end{array}$ & $\begin{array}{l}L_{0} *, L_{100}, L_{500}, L_{1000}, L_{\infty} \\
L_{250}, L_{750}, L_{1250}, L_{1500}\end{array}$ & $\begin{array}{l}L_{K}> \\
L_{K}= \\
L_{K}<\end{array}$ & $\begin{array}{l}L_{0} *, L_{250}, L_{1000}, L_{1250}, L_{1500} \\
L_{750} \\
L_{100}, L_{500}, L_{\infty}\end{array}$ & $\begin{array}{l}L_{K}> \\
L_{K}= \\
L_{K}<\end{array}$ & $\begin{array}{l}L_{0} *, L_{100}, L_{250}, L_{1250}, L_{\infty} \\
L_{1500} \\
L_{500}, L_{750}, L_{1000}\end{array}$ \\
\hline $\mathrm{D}=\mathbf{5 0}$ & $\begin{array}{l}L_{K}> \\
L_{K}= \\
L_{K}<\end{array}$ & $\begin{array}{l}L_{0} *, L_{1000} \\
L_{100}, L_{250}, L_{500}, L_{750}, L_{1250}, L_{1500}, L_{\infty}\end{array}$ & $\begin{array}{l}L_{K}> \\
L_{K}= \\
L_{K}<\end{array}$ & $\begin{array}{l}L_{0} *, L_{100}, L_{250}, L_{500}, L_{750}, L_{1500}, L_{\infty} \\
L_{1250} \\
L_{1000}\end{array}$ & $\begin{array}{l}L_{K}> \\
L_{K}= \\
L_{K}<\end{array}$ & $\begin{array}{l}L_{0} *, L_{250}, L_{500}, L_{750}, L_{1250}, L_{1500} \\
L_{100}, L_{1000}, L_{\infty}\end{array}$ \\
\hline
\end{tabular}


Table 35

$f_{5}$, vertical approach, MaxFEs $=100,000$, CRS4EAs

\begin{tabular}{|c|c|c|c|c|c|c|}
\hline & & $\mathrm{SN}=24$ & & $\mathrm{SN}=\mathbf{5 0}$ & & $\mathrm{SN}=100$ \\
\hline & $L_{K}>$ & $L_{0} *, L_{100}, L_{250}, L_{500}, L_{750}, L_{1000}, L_{1250}$ & $L_{K}>$ & $L_{0}, L_{100}, L_{1000}, L_{1500}, L_{\infty}$ & $L_{K}>$ & $L_{1000}, L_{1250}, L_{1500}, L_{\infty}$ \\
\hline$D=2$ & $L_{K}=$ & & $L_{K}=$ & $L_{500}$ & $L_{K}=$ & $L_{100}$ \\
\hline \multirow[t]{2}{*}{$D=5$} & $\begin{array}{l}L_{K}> \\
L_{K}=\end{array}$ & $L_{0} *$ & $\begin{array}{l}L_{K}> \\
L_{K}> \\
L_{K}=\end{array}$ & $\frac{L_{250}, L_{750}, L_{1250}}{L_{0} *, L_{100}}$ & $\begin{array}{l}L_{K}< \\
L_{K}> \\
L_{K}=\end{array}$ & $\begin{array}{l}L_{0}, L_{250}, L_{500}, L_{750} \\
L_{0} *, L_{100} \\
L_{250}\end{array}$ \\
\hline & $L_{K}^{n}<$ & $\begin{array}{l}L_{100}, \quad L_{250 *}, \quad L_{500 *}, \quad L_{750} *, \quad L_{1000 *}, \\
L_{1250}, L_{1500 *,} L_{\infty} *\end{array}$ & $L_{K}<$ & $\begin{array}{l}L_{250}, L_{500}, L_{750}, L_{1000} *, L_{1250} *, L_{1500} * \text {, } \\
L_{\infty} *\end{array}$ & $L_{K}<$ & $L_{500}, L_{750}, L_{1000}, L_{1250}, L_{1500}, L_{\infty}$ \\
\hline \multirow[t]{2}{*}{$D=10$} & $\begin{array}{l}L_{K}> \\
L_{K}=\end{array}$ & $L_{0} *, L_{100}$ & $\begin{array}{l}L_{K}> \\
L_{K}=\end{array}$ & $\begin{array}{l}L_{0} *, L_{100} \\
L_{250}\end{array}$ & $\begin{array}{l}L_{K}> \\
L_{K}=\end{array}$ & $\begin{array}{l}L_{0} *, L_{100} *, L_{250}, L_{750}, L_{1000}, L_{1500} \\
L_{500}\end{array}$ \\
\hline & $L_{K}<$ & $\begin{array}{l}L_{250}, L_{500}, L_{750} *, L_{1000}, L_{1250}, L_{1500}, \\
L_{\infty} *\end{array}$ & $L_{K}<$ & $L_{500}, L_{750}, L_{1000}, L_{1250}, L_{1500}, L_{\infty}$ & $L_{K}<$ & $L_{1250}, L_{\infty}$ \\
\hline \multirow[b]{2}{*}{$\mathbf{D}=\mathbf{3 0}$} & $L_{K}>$ & $L_{0} *, L_{100}, L_{1250}, L_{1500}, L_{\infty}$ & $L_{K}>$ & $\begin{array}{l}L_{0} *, L_{100}, L_{250}, L_{500}, L_{1000}, L_{1250}, \\
L_{1500}, L_{\infty}\end{array}$ & $L_{K}>$ & $L_{0} *, L_{250}, L_{500}, L_{750}, L_{1250}, L_{\infty}$ \\
\hline & $\begin{array}{l}L_{K}= \\
L_{K}<\end{array}$ & $L_{250}, L_{500}, L_{750}, L_{1000}$ & $\begin{array}{l}L_{K}= \\
L_{K}<\end{array}$ & $L_{750}$ & $\begin{array}{l}L_{K}= \\
L_{K}<\end{array}$ & $\begin{array}{l}L_{100}, L_{1500} \\
L_{1000}\end{array}$ \\
\hline \multirow[t]{2}{*}{$\mathrm{D}=\mathbf{5 0}$} & $\begin{array}{l}L_{K}> \\
L_{K}=\end{array}$ & $L_{0} *, L_{1250}$ & $\begin{array}{l}L_{K}> \\
L_{K}=\end{array}$ & $\begin{array}{l}L_{0} *, L_{250}, L_{1500} \\
L_{1250}\end{array}$ & $\begin{array}{l}L_{K}> \\
L_{K}=\end{array}$ & $L_{0} *$ \\
\hline & $L_{K}<$ & $L_{100}, L_{250}, L_{500}, L_{750}, L_{1000}, L_{1500}, L_{\infty}$ & $L_{K}<$ & $L_{100}, L_{500}, L_{750}, L_{1000}, L_{\infty}$ & $L_{K}<$ & $\begin{array}{l}L_{100}, L_{250}, L_{500}, L_{750}, L_{1000}, L_{1250}, \\
L_{1500}, L_{\infty}\end{array}$ \\
\hline
\end{tabular}

Table 36

Vertical approach. MaxFEs $=250,000$

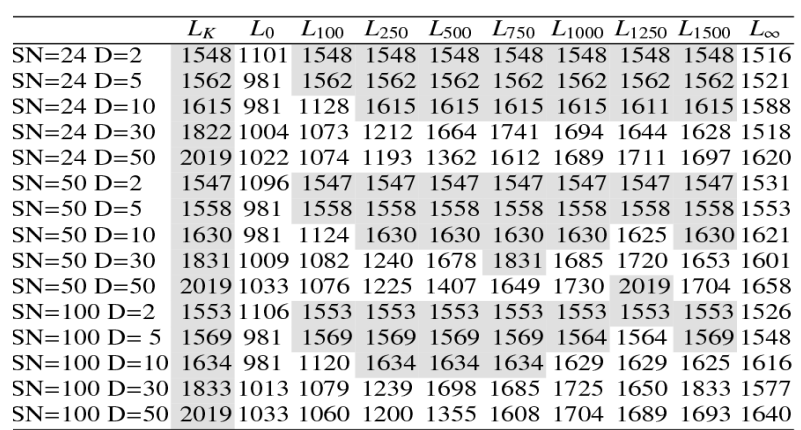

(a) Vertical approach, $f_{1}$, MaxFEs $=250,000$

\begin{tabular}{lcccccccccc}
\hline & $L_{K}$ & $L_{0}$ & $L_{100}$ & $L_{250}$ & $L_{500}$ & $L_{750}$ & $L_{1000}$ & $L_{1250}$ & $L_{1500}$ & $L_{\infty}$ \\
\hline $\mathrm{SN}=24 \mathrm{D}=2$ & 1994981 & 1648 & 1715 & 1465 & 1593 & 1366 & 1512 & 1459 & 1267 \\
$\mathrm{SN}=24 \mathrm{D}=5$ & 1982981 & 1695 & 1637 & 1598 & 1517 & 1502 & 1434 & 1420 & 1233 \\
$\mathrm{SN}=24 \mathrm{D}=10$ & 1967981 & 1583 & 1591 & 1558 & 1553 & 1547 & 1460 & 1461 & 1299 \\
$\mathrm{SN}=24 \mathrm{D}=30$ & 1941 & 981 & 1145 & 1431 & 1548 & 1575 & 1583 & 1561 & 1604 & 1632 \\
$\mathrm{SN}=24 \mathrm{D}=50$ & 1998981 & 1119 & 1539 & 1530 & 1614 & 1547 & 1557 & 1543 & 1572 \\
$\mathrm{SN}=50 \mathrm{D}=2$ & 1987981 & 1616 & 1699 & 1610 & 1579 & 1381 & 1459 & 1488 & 1200 \\
$\mathrm{SN}=50 \mathrm{D}=5$ & 1973981 & 1679 & 1638 & 1547 & 1513 & 1502 & 1446 & 1471 & 1250 \\
$\mathrm{SN}=50 \mathrm{D}=10$ & 1971981 & 1570 & 1971 & 1550 & 1557 & 1566 & 1506 & 1495 & 1304 \\
$\mathrm{SN}=50 \mathrm{D}=30$ & 1980981 & 1152 & 1471 & 1616 & 1980 & 1619 & 1503 & 1644 & 1533 \\
$\mathrm{SN}=50 \mathrm{D}=50$ & 2010981 & 1144 & 1522 & 1558 & 1555 & 1575 & 2010 & 1577 & 1578 \\
$\mathrm{SN}=100 \mathrm{D}=2$ & 1991981 & 1991 & 1697 & 1610 & 1562 & 1529 & 1473 & 1441 & 1216 \\
$\mathrm{SN}=100 \mathrm{D}=5$ & 1976981 & 1678 & 1976 & 1599 & 1531 & 1530 & 1467 & 1445 & 1292 \\
$\mathrm{SN}=100 \mathrm{D}=10$ & 1973981 & 1581 & 1590 & 1973 & 1512 & 1568 & 1503 & 1497 & 1297 \\
$\mathrm{SN}=100 \mathrm{D}=30$ & 1966981 & 1170 & 1470 & 1535 & 1584 & 1629 & 1583 & 1966 & 1582 \\
$\mathrm{SN}=100 \mathrm{D}=50$ & 2007981 & 1124 & 1475 & 1567 & 1557 & 1588 & 1548 & 1547 & 1607 \\
\hline
\end{tabular}

(b) Vertical approach, $f_{2}$, MaxFEs $=250,000$

\begin{tabular}{lcccccccccc}
\hline & $L_{K}$ & $L_{0}$ & $L_{100}$ & $L_{250}$ & $L_{500}$ & $L_{750}$ & $L_{1000}$ & $L_{1250}$ & $L_{1500}$ & $L_{\infty}$ \\
\hline $\mathrm{SN}=24 \mathrm{D}=2$ & 1550 & 1048 & 1550 & 1550 & 1550 & 1550 & 1550 & 1550 & 1550 & 1550 \\
$\mathrm{SN}=24 \mathrm{D}=5$ & 1561 & 981 & 1561 & 1561 & 1561 & 1561 & 1561 & 1561 & 1561 & 1530 \\
$\mathrm{SN}=24 \mathrm{D}=10$ & 1903 & 981 & 1529 & 1535 & 1529 & 1535 & 1519 & 1503 & 1498 & 1467 \\
$\mathrm{SN}=24 \mathrm{D}=30$ & 1923 & 981 & 1263 & 1460 & 1520 & 1559 & 1599 & 1538 & 1589 & 1569 \\
$\mathrm{SN}=24 \mathrm{D}=50$ & 1937 & 981 & 1106 & 1353 & 1604 & 1594 & 1598 & 1615 & 1611 & 1599 \\
$\mathrm{SN}=50 \mathrm{D}=2$ & 1554 & 1017 & 1554 & 1554 & 1554 & 1554 & 1554 & 1554 & 1554 & 1554 \\
$\mathrm{SN}=50 \mathrm{D}=5$ & 1561 & 981 & 1561 & 1561 & 1561 & 1561 & 1561 & 1561 & 1561 & 1535 \\
$\mathrm{SN}=50 \mathrm{D}=10$ & 1917 & 981 & 1523 & 1917 & 1543 & 1533 & 1528 & 1502 & 1507 & 1466 \\
$\mathrm{SN}=50 \mathrm{D}=30$ & 1934 & 981 & 1243 & 1487 & 1523 & 1934 & 1597 & 1577 & 1573 & 1585 \\
$\mathrm{SN}=50 \mathrm{D}=50$ & 1958 & 981 & 1111 & 1446 & 1586 & 1607 & 1582 & 1958 & 1606 & 1623 \\
$\mathrm{SN}=100 \mathrm{D}=2$ & 1558 & 1033 & 1558 & 1558 & 1558 & 1558 & 1558 & 1558 & 1558 & 1558 \\
$\mathrm{SN}=100 \mathrm{D}=5$ & 1566 & 981 & 1566 & 1566 & 1566 & 1566 & 1566 & 1566 & 1556 & 1556 \\
$\mathrm{SN}=100 \mathrm{D}=10$ & 1867 & 981 & 1550 & 1555 & 1867 & 1550 & 1540 & 1488 & 1508 & 1462 \\
$\mathrm{SN}=100 \mathrm{D}=30$ & 1940 & 981 & 1235 & 1475 & 1546 & 1569 & 1577 & 1597 & 1940 & 1580 \\
$\mathrm{SN}=100 \mathrm{D}=50$ & 1941 & 981 & 1108 & 1397 & 1555 & 1592 & 1606 & 1610 & 1613 & 1598 \\
\hline
\end{tabular}

(c) Vertical approach, $f_{3}$, MaxFEs $=250,000$ \begin{tabular}{lcccccccccc}
\hline & $L_{K}$ & $L_{0}$ & $L_{100}$ & $L_{250}$ & $L_{500}$ & $L_{750}$ & $L_{1000}$ & $L_{1250}$ & $L_{1500}$ & $L_{\infty}$ \\
\hline $\mathrm{SN}=24 \mathrm{D}=2$ & 1558981 & 1558 & 1558 & 1558 & 1558 & 1558 & 1558 & 1558 & 1558
\end{tabular} $\begin{array}{llllllllllll}\mathrm{SN}=24 \mathrm{D}=5 & 1558981 & 1558 & 1558 & 1558 & 1558 & 1558 & 1558 & 1558 & 1558\end{array}$ $\begin{array}{llllllllllll}\mathrm{SN}=24 & \mathrm{D}=10 & 1558981 & 1558 & 1558 & 1558 & 1558 & 1558 & 1558 & 1558 & 1558\end{array}$ $\begin{array}{lllllllllllll}\mathrm{SN}=24 \mathrm{D}=30 & 1577 & 981 & 1405 & 1577 & 1577 & 1577 & 1577 & 1577 & 1577 & 1577\end{array}$

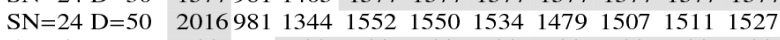
$\begin{array}{lllllllllll}\mathrm{SN}=50 & \mathrm{D}=2 & 1558981 & 1558 & 1558 & 1558 & 1558 & 1558 & 1558 & 1558 & 1558\end{array}$ $\begin{array}{lllllllllll}\mathrm{SN}=50 & \mathrm{D}=5 & 1558981 & 1558 & 1558 & 1558 & 1558 & 1558 & 1558 & 1558 & 1558\end{array}$

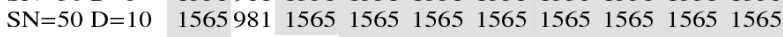
$\begin{array}{lllllllllllll}\mathrm{SN}=50 & \mathrm{D}=30 & 1586981 & 1415 & 1586 & 1586 & 1586 & 1586 & 1586 & 1586 & 1586\end{array}$ $\begin{array}{llllllllllll}\mathrm{SN}=50 \mathrm{D}=50 & 1586981 & 1415 & 1586 & 1586 & 1586 & 1586 & 1586 & 1586 & 1586\end{array}$ $\mathrm{SN}=100 \mathrm{D}=2 \quad 1565981 \quad 1565 \quad 1565 \quad 1565 \quad 1565 \quad 1565 \quad 1565 \quad 1565 \quad 1565$ $\mathrm{SN}=100 \mathrm{D}=5 \quad 1565981 \quad 15651565 \quad 1565 \quad 1565 \quad 15651565 \quad 1565 \quad 1565$

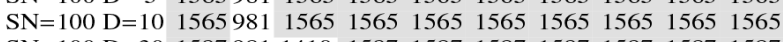

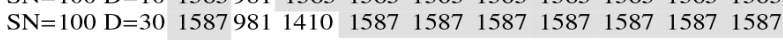
$\mathrm{SN}=100 \mathrm{D}=50 \quad 2011981 \quad 1351 \quad 1558 \quad 1509 \quad 1482 \quad 1530 \quad 1525 \quad 1540 \quad 1514$

(d) Vertical approach, $f_{4}$, MaxFEs $=250,000$

\begin{tabular}{cccccccccc}
\hline$L_{K}$ & $L_{0}$ & $L_{100}$ & $L_{250}$ & $L_{500}$ & $L_{750}$ & $L_{1000}$ & $L_{1250}$ & $L_{1500}$ & $L_{\infty}$
\end{tabular} \begin{tabular}{llllllllllllll}
\hline $\mathrm{SN}=24 \mathrm{D}=2$ & 1914 & 1494 & 1508 & 1495 & 1485 & 1492 & 1437 & 1431 & 1438 & 1306
\end{tabular}

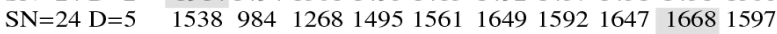
$\mathrm{SN}=24 \mathrm{D}=10 \quad 1582 \quad 981 \quad 12721476 \quad 1547 \quad 1610 \quad 1608 \quad 1621 \quad 1649 \quad 1655$

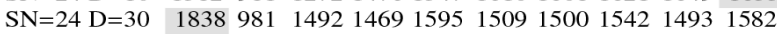
$\begin{array}{llllllllllll}\mathrm{SN}=24 & \mathrm{D}=50 & 1933 & 981 & 1493 & 1477 & 1482 & 1506 & 1507 & 1512 & 1578 & 1532\end{array}$

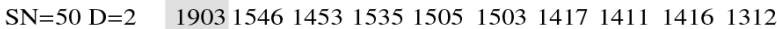
\begin{tabular}{ll|llllllllll}
$\mathrm{SN}=50$ & $\mathrm{D}=5$ & 1625 & 982 & 1230 & 1439 & 1562 & 1627 & 1620 & 1625 & 1653 & 1637
\end{tabular}

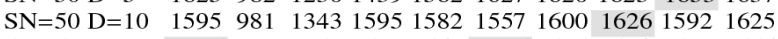

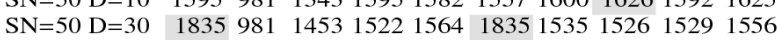

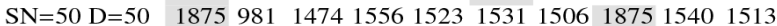
$\mathrm{SN}=100 \mathrm{D}=2 \quad 17491551 \quad 17491491 \quad 1495 \quad 1503 \quad 1500 \quad 1465 \quad 1443 \quad 1304$

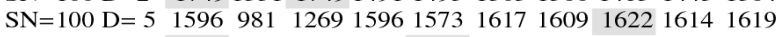
$\begin{array}{llllllllllll}\mathrm{SN}=100 & \mathrm{D}=10 & 1704 & 981 & 1287 & 1514 & 1704 & 1618 & 1623 & 1583 & 1588 & 1601\end{array}$

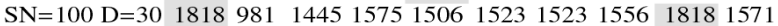
SN=100 D=50 $1888981 \quad 15291551 \quad 1482 \quad 1506 \quad 1506 \quad 1523 \quad 1509 \quad 1527$

(e) Vertical approach, $f_{5}$, MaxFEs $=250,000$ 
Tables 37-41 show more clearly the differences found between $L_{k}$ and other 9 fixed 'limit' values on all 5 optimisation problems. As mentioned before, the better alternatives were found only for problem $f_{5}$, when the dimensions were either 5 or 10 but the 'limit' values were not significantly better than $L_{k}$. As in NHST analysis, the CRS4EAs also showed that whenever sufficiently larger numbers of function evaluations were available, Karaboga's 'limit' setting was an appropriate choice. In this approach, both methods, NHST and CRS4EAs, appeared equally conservative (compare Tables 10-14 with Tables 37-41).

\subsubsection{Experiment 3: Horizontal Approach $-10^{-6}-$ MaxFEs $=1,000,000$}

In the horizontal approach, there were fixed 'limit' values that found (sub-)optimal solutions in fewer fitness evaluations than $L_{k}$ for all optimisation problems. Tables 42(a)-42(e) show the ratings for every optimisation problem and every setting of $S N$ and $D$. All 'limit' values reached the minimum rating deviation value of 50 rating points and the better rating values are again highlighted with light grey colour.

For $f_{1}$, better alternatives than $L_{k}$ were available for the smaller population size $S N=24$ and for the greater population size $S N=100$, whereas for $S N=50, L_{k}$ was only worse for $D=5$ and $D=50$ and better for all other dimension values. For $f_{2}, L_{k}$ was the worst value for all population sizes and dimensions, except for $S N=50$ and $D=10, S N=100$ and $D=10, S N=50$ and $D=30$, and $S N$ $=50$ and $D=50$. For $f_{3}, L_{k}$ was the better value only for $S N=24$ and $D=2$ and $S N=24$ and $D=50$, but for other settings there were better alternatives. For $f_{4}, L_{k}$ always had a better alternative and was always worse than at

Table 37

$f_{1}$, vertical approach, MaxFEs $=250,000$, CRS4EAs

\begin{tabular}{|c|c|c|c|c|c|c|}
\hline & & $\mathrm{SN}=24$ & & $\mathrm{SN}=\mathbf{5 0}$ & & $S N=100$ \\
\hline$D=2$ & $\begin{array}{l}L_{K}> \\
L_{K}= \\
L_{K}<\end{array}$ & $\begin{array}{l}L_{0 *,} L_{\infty} \\
L_{100}, L_{250}, L_{500}, L_{750}, L_{1000}, L_{1250} \\
L_{1500}\end{array}$ & $\begin{array}{l}L_{K}> \\
L_{K}= \\
L_{K}<\end{array}$ & $\begin{array}{l}L_{0} *, L_{\infty} \\
L_{100}, L_{250}, L_{500}, L_{750}, L_{1000}, L_{1250}, \\
L_{1500}\end{array}$ & $\begin{array}{l}L_{K}> \\
L_{K}= \\
L_{K}<\end{array}$ & $\begin{array}{l}L_{0} *, L_{\infty} \\
L_{100}, L_{250}, L_{500}, L_{750}, L_{1000}, L_{1250}, \\
L_{1500}\end{array}$ \\
\hline$D=5$ & $\begin{array}{l}L_{K}> \\
L_{K}= \\
L_{K}<\end{array}$ & $\begin{array}{l}L_{0 *}, L_{\infty} \\
L_{100}, L_{250}, L_{500}, L_{750}, L_{1000}, L_{1250} \\
L_{1500}\end{array}$ & $\begin{array}{l}L_{K}> \\
L_{K}= \\
L_{K}<\end{array}$ & $\begin{array}{l}L_{0} *, L_{\infty} \\
L_{100}, L_{250}, L_{500}, L_{750}, L_{1000}, L_{1250}, \\
L_{1500}\end{array}$ & $\begin{array}{l}L_{K}> \\
L_{K}= \\
L_{K}<\end{array}$ & $\begin{array}{l}L_{0} *, L_{1000}, L_{1250}, L_{\infty} \\
L_{100}, L_{250}, L_{500}, L_{750}, L_{1500}\end{array}$ \\
\hline$D=10$ & $\begin{array}{l}L_{K}> \\
L_{K}= \\
L_{K}<\end{array}$ & $\begin{array}{l}L_{0} *, L_{100} *, L_{1250}, L_{\infty} \\
L_{250}, L_{500}, L_{750}, L_{1000}, L_{1500}\end{array}$ & $\begin{array}{l}L_{K}> \\
L_{K}= \\
L_{K}<\end{array}$ & $\begin{array}{l}L_{0} *, L_{100} *, L_{1250}, L_{\infty} \\
L_{250}, L_{500}, L_{750}, L_{1000}, L_{1500}\end{array}$ & $\begin{array}{l}L_{K}> \\
L_{K}= \\
L_{K}<\end{array}$ & $\begin{array}{l}L_{0} *, L_{100} *, L_{1000}, L_{1250}, L_{1500}, L_{\infty} \\
L_{250}, L_{500}, L_{750}\end{array}$ \\
\hline $\mathrm{D}=\mathbf{3 0}$ & $\begin{array}{l}L_{K}> \\
L_{K}= \\
L_{K}<\end{array}$ & $\begin{array}{l}L_{0} *, L_{100} *, L_{250} *, L_{500}, L_{750}, L_{1000} \\
L_{1250}, L_{1500}, L_{\infty} *\end{array}$ & $\begin{array}{l}L_{K}> \\
L_{K}= \\
L_{K}<\end{array}$ & $\begin{array}{l}L_{0} *, L_{100} *, L_{250} *, L_{500}, L_{1000}, L_{1250}, \\
L_{1500}, L_{\infty} * \\
L_{750}\end{array}$ & $\begin{array}{l}L_{K}> \\
L_{K}= \\
L_{K}<\end{array}$ & $\begin{array}{l}L_{0} *, L_{100} *, L_{250} *, L_{500}, L_{750}, L_{1000}, \\
L_{1250}, L_{\infty} * \\
L_{1500}\end{array}$ \\
\hline$D=50$ & $\begin{array}{l}L_{K}> \\
L_{K}= \\
L_{K}<\end{array}$ & $\begin{array}{l}L_{0} *, L_{100} *, L_{250} *, L_{500} *, L_{750} *, L_{1000} * \\
L_{1250} *, L_{1500} *, L_{\infty} *\end{array}$ & $\begin{array}{l}L_{K}> \\
L_{K}= \\
L_{K}<\end{array}$ & $\begin{array}{l}L_{0} *, L_{100} *, L_{250} *, L_{500} *, L_{750} *, L_{1000} * \\
L_{1500 *, L_{\infty} *} \\
L_{1250}\end{array}$ & $\begin{array}{l}L_{K}> \\
L_{K}= \\
L_{K}<\end{array}$ & $\begin{array}{l}L_{0} *, L_{100} *, L_{250} *, L_{500} *, L_{750} *, L_{1000} * \\
L_{1250} *, L_{1500} *, L_{\infty} *\end{array}$ \\
\hline
\end{tabular}

Table 38

$\mathrm{f}_{2}$, vertical approach, MaxFEs $=250,000$, CRS4EAs

\begin{tabular}{|c|c|c|c|c|c|c|}
\hline & & $\mathrm{SN}=24$ & & $\mathrm{SN}=50$ & & $\mathrm{SN}=100$ \\
\hline$D=2$ & $\begin{array}{l}L_{K}> \\
L_{K}= \\
L_{K}<\end{array}$ & $\begin{array}{l}L_{0} *, L_{100} *, L_{250} *, L_{500} *, L_{750} *, L_{1000} * \\
L_{1250}, L_{1500}, L_{\infty} *\end{array}$ & $\begin{array}{l}L_{K}> \\
L_{K}= \\
L_{K}<\end{array}$ & $\begin{array}{l}L_{0} *, L_{100} *, L_{250} *, L_{500} *, L_{750} *, L_{1000} * \\
L_{1250} *, L_{1500} *, L_{\infty} *\end{array}$ & $\begin{array}{l}L_{K}> \\
L_{K}= \\
L_{K}<\end{array}$ & $\begin{array}{l}L_{0} *, L_{250} *, L_{500} *, L_{750} *, L_{1000} *, L_{1250} * \\
L_{1500 *, L_{\infty} *} \\
L_{100}\end{array}$ \\
\hline$D=5$ & $\begin{array}{l}L_{K}> \\
L_{K}= \\
L_{K}<\end{array}$ & $\begin{array}{l}L_{0} *, L_{100} *, L_{250} *, L_{500} *, L_{750} *, L_{1000} * \\
L_{1250}, L_{1500} *, L_{\infty} *\end{array}$ & $\begin{array}{l}L_{K}> \\
L_{K}= \\
L_{K}<\end{array}$ & $\begin{array}{l}L_{0} *, L_{100} *, L_{250} *, L_{500} *, L_{750} *, L_{1000} * \\
L_{1250} *, L_{1500} *, L_{\infty} *\end{array}$ & $\begin{array}{l}L_{K}> \\
L_{K}= \\
L_{K}<\end{array}$ & $\begin{array}{l}L_{0} *, L_{100} *, L_{500} *, L_{750} *, L_{1000} *, L_{1250} * \\
L_{1500}, L_{\infty} * \\
L_{250}\end{array}$ \\
\hline$D=10$ & $\begin{array}{l}L_{K}> \\
L_{K}= \\
L_{K}<\end{array}$ & $\begin{array}{l}L_{0} *, L_{100} *, L_{250} *, L_{500} *, L_{750} *, L_{1000} * \\
L_{1250}, L_{1500} *, L_{\infty} *\end{array}$ & $\begin{array}{l}L_{K}> \\
L_{K}= \\
L_{K}<\end{array}$ & $\begin{array}{l}L_{0} *, L_{100} *, L_{500} *, L_{750} *, L_{1000} *, L_{1250} * \\
L_{1500 *, L_{\infty} *} \\
L_{250}\end{array}$ & $\begin{array}{l}L_{K}> \\
L_{K}= \\
L_{K}<\end{array}$ & $\begin{array}{l}L_{0} *, L_{100} *, L_{250} *, L_{750} *, L_{1000} *, L_{1250} * \\
L_{1500}, L_{\infty} * \\
L_{500}\end{array}$ \\
\hline$D=30$ & $\begin{array}{l}L_{K}> \\
L_{K}= \\
L_{K}<\end{array}$ & $\begin{array}{l}L_{0} *, L_{100} *, L_{250} *, L_{500} *, L_{750} *, L_{1000} * \\
L_{1250}, L_{1500} *, L_{\infty} *\end{array}$ & $\begin{array}{l}L_{K}> \\
L_{K}= \\
L_{K}<\end{array}$ & $\begin{array}{l}L_{0} *, L_{100} *, L_{250} *, L_{500} * L_{1000} *, L_{1250} * \\
L_{1500} *, L_{\infty} * \\
L_{750}\end{array}$ & $\begin{array}{l}L_{K}> \\
L_{K}= \\
L_{K}<\end{array}$ & $\begin{array}{l}L_{0} *, L_{100} *, L_{250} *, L_{500} *, L_{750} *, L_{1000} * \\
L_{1250} *, L_{\infty} * \\
L_{1500}\end{array}$ \\
\hline$D=50$ & $\begin{array}{l}L_{K}> \\
L_{K}= \\
L_{K}<\end{array}$ & $\begin{array}{l}L_{0} *, L_{100} *, L_{250} *, L_{500} *, L_{750} *, L_{1000} * \\
L_{1250}, L_{1500} *, L_{\infty} *\end{array}$ & $\begin{array}{l}L_{K}> \\
L_{K}= \\
L_{K}<\end{array}$ & $\begin{array}{l}L_{0} *, L_{100} *, L_{250} *, L_{500} *, L_{750} *, L_{1000} * \\
L_{1500} *, L_{\infty} * \\
L_{1250}\end{array}$ & $\begin{array}{l}L_{K}> \\
L_{K}= \\
L_{K}<\end{array}$ & $\begin{array}{l}L_{0} *, L_{100} *, L_{250} *, L_{500} *, L_{750} *, L_{1000} * \\
L_{1250} *, L_{1500} *, L_{\infty} *\end{array}$ \\
\hline
\end{tabular}


Table 39

$f_{3}$, vertical approach, MaxFEs $=250,000$, CRS4EAs

\begin{tabular}{|c|c|c|c|c|c|c|}
\hline & & $\mathrm{SN}=24$ & & $\mathrm{SN}=50$ & & $\mathrm{SN}=100$ \\
\hline$D=2$ & $\begin{array}{l}L_{K}> \\
L_{K}= \\
L_{K}<\end{array}$ & $\begin{array}{l}L_{0} * \\
L_{100}, L_{250}, L_{500}, L_{750}, L_{1000}, L_{1250}, \\
L_{1500}, L_{\infty}\end{array}$ & $\begin{array}{l}L_{K}> \\
L_{K}= \\
L_{K}<\end{array}$ & $\begin{array}{l}L_{0} * \\
L_{100}, L_{250}, L_{500}, L_{750}, L_{1000}, L_{1250}, \\
L_{1500}, L_{\infty}\end{array}$ & $\begin{array}{l}L_{K}> \\
L_{K}= \\
L_{K}<\end{array}$ & $\begin{array}{l}L_{0} * \\
L_{100}, L_{250}, L_{500}, L_{750}, L_{1000}, L_{1250}, \\
L_{1500}, L_{\infty}\end{array}$ \\
\hline$D=5$ & $\begin{array}{l}L_{K}> \\
L_{K}= \\
L_{K}<\end{array}$ & $\begin{array}{l}L_{0} *, L_{\infty} \\
L_{100}, L_{250}, L_{500}, L_{750}, L_{1000}, L_{1250}, \\
L_{1500}\end{array}$ & $\begin{array}{l}L_{K}> \\
L_{K}= \\
L_{K}<\end{array}$ & $\begin{array}{l}L_{0} *, L_{\infty} \\
L_{100}, L_{250}, L_{500}, L_{750}, L_{1000}, L_{1250}, \\
L_{1500}\end{array}$ & $\begin{array}{l}L_{K}> \\
L_{K}= \\
L_{K}<\end{array}$ & $\begin{array}{l}L_{0} * \\
L_{100}, L_{250}, L_{500}, L_{750}, L_{1000}, L_{1250}, \\
L_{1500}, L_{\infty}\end{array}$ \\
\hline$D=10$ & $\begin{array}{l}L_{K}> \\
L_{K}= \\
L_{K}<\end{array}$ & $\begin{array}{l}L_{0} *, L_{100} *, L_{250} *, L_{500} *, L_{750} *, L_{1000} * \\
L_{1250} *, L_{1500} *, L_{\infty} *\end{array}$ & $\begin{array}{l}L_{K}> \\
L_{K}= \\
L_{K}<\end{array}$ & $\begin{array}{l}L_{0} *, L_{100} *, L_{500} *, L_{750} *, L_{1000} *, L_{1250} * \\
L_{1500} *, L_{\infty} * \\
L_{250}\end{array}$ & $\begin{array}{l}L_{K}> \\
L_{K}= \\
L_{K}<\end{array}$ & $\begin{array}{l}L_{0} *, L_{100} *, L_{250} *, L_{750} *, L_{1000} *, L_{1250} * \\
L_{1500}, L_{\infty} * \\
L_{500}\end{array}$ \\
\hline$D=30$ & $\begin{array}{l}L_{K}> \\
L_{K}= \\
L_{K}<\end{array}$ & $\begin{array}{l}L_{0} *, L_{100} *, L_{250} *, L_{500} *, L_{750} *, L_{1000} * \\
L_{1250}, L_{1500 *,}, L_{\infty} *\end{array}$ & $\begin{array}{l}L_{K}> \\
L_{K}= \\
L_{K}<\end{array}$ & $\begin{array}{l}L_{0} *, L_{100} *, L_{250} *, L_{500} *, L_{1000} *, L_{1250} * \\
L_{1500} *, L_{\infty} * \\
L_{750}\end{array}$ & $\begin{array}{l}L_{K}> \\
L_{K}= \\
L_{K}<\end{array}$ & $\begin{array}{l}L_{0} *, L_{100} *, L_{250} *, L_{500} *, L_{750} *, L_{1000} * \\
L_{1250 *, L_{\infty}} * \\
L_{1500}\end{array}$ \\
\hline$D=50$ & $\begin{array}{l}L_{K}> \\
L_{K}= \\
L_{K}<\end{array}$ & $\begin{array}{l}L_{0} *, L_{100} *, L_{250} *, L_{500} *, L_{750} *, L_{1000} * \\
L_{1250} *, L_{1500} *, L_{\infty} *\end{array}$ & $\begin{array}{l}L_{K}> \\
L_{K}= \\
L_{K}<\end{array}$ & $\begin{array}{l}L_{0} *, L_{100} *, L_{250} *, L_{500} *, L_{750} *, L_{1000} * \\
L_{1500} *, L_{\infty} * \\
L_{1250}\end{array}$ & $\begin{array}{l}L_{K}> \\
L_{K}= \\
L_{K}<\end{array}$ & $\begin{array}{l}L_{0} *, L_{100} *, L_{250} *, L_{500} *, L_{750} *, L_{1000} * \\
L_{1250} *, L_{1500} *, L_{\infty} *\end{array}$ \\
\hline
\end{tabular}

Table 40

$f_{4}$, vertical approach, MaxFEs $=250,000$, CRS4EAs

\begin{tabular}{|c|c|c|c|c|c|c|}
\hline & & $\mathrm{SN}=24$ & & $\mathrm{SN}=50$ & & $S N=100$ \\
\hline$D=2$ & $\begin{array}{l}L_{K}> \\
L_{K}= \\
L_{K}<\end{array}$ & $\begin{array}{l}L_{0 *} \\
L_{100}, L_{250}, L_{500}, L_{750}, L_{1000}, L_{1250}, \\
L_{1500}, L_{\infty}\end{array}$ & $\begin{array}{l}L_{K}> \\
L_{K}= \\
L_{K}<\end{array}$ & $\begin{array}{l}L_{0} * \\
L_{100}, L_{250}, L_{500}, L_{750}, L_{1000}, L_{1250}, \\
L_{1500}, L_{\infty}\end{array}$ & $\begin{array}{l}L_{K}> \\
L_{K}= \\
L_{K}<\end{array}$ & $\begin{array}{l}L_{0} * \\
L_{100}, L_{250}, L_{500}, L_{750}, L_{1000}, L_{1250}, \\
L_{1500}, L_{\infty}\end{array}$ \\
\hline$D=5$ & $\begin{array}{l}L_{K}> \\
L_{K}= \\
L_{K}<\end{array}$ & $\begin{array}{l}L_{0} * \\
L_{100}, L_{250}, L_{500}, L_{750}, L_{1000}, L_{1250}, \\
L_{1500}, L_{\infty}\end{array}$ & $\begin{array}{l}L_{K}> \\
L_{K}= \\
L_{K}<\end{array}$ & $\begin{array}{l}L_{0} * \\
L_{100}, L_{250}, L_{500}, L_{750}, L_{1000}, L_{1250}, \\
L_{1500}, L_{\infty}\end{array}$ & $\begin{array}{l}L_{K}> \\
L_{K}= \\
L_{K}<\end{array}$ & $\begin{array}{l}L_{0} * \\
L_{100}, L_{250}, L_{500}, L_{750}, L_{1000}, L_{1250}, \\
L_{1500}, L_{\infty}\end{array}$ \\
\hline$D=10$ & $\begin{array}{l}L_{K}> \\
L_{K}= \\
L_{K}<\end{array}$ & $\begin{array}{l}L_{0} * \\
L_{100}, L_{250}, L_{500}, L_{750}, L_{1000}, L_{1250}, \\
L_{1500}, L_{\infty}\end{array}$ & $\begin{array}{l}L_{K}> \\
L_{K}= \\
L_{K}<\end{array}$ & $\begin{array}{l}L_{0} * \\
L_{100}, L_{250}, L_{500}, L_{750}, L_{1000}, L_{1250} \\
L_{1500}, L_{\infty}\end{array}$ & $\begin{array}{l}L_{K}> \\
L_{K}= \\
L_{K}<\end{array}$ & $\begin{array}{l}L_{0} * \\
L_{100}, L_{250}, L_{500}, L_{750}, L_{1000}, L_{1250}, \\
L_{1500}, L_{\infty}\end{array}$ \\
\hline$D=30$ & $\begin{array}{l}L_{K}> \\
L_{K}= \\
L_{K}<\end{array}$ & $\begin{array}{l}L_{0} *, L_{100} \\
L_{250}, L_{500}, L_{750}, L_{1000}, L_{1250}, L_{1500}, L_{\infty}\end{array}$ & $\begin{array}{l}L_{K}> \\
L_{K}= \\
L_{K}<\end{array}$ & $\begin{array}{l}L_{0} *, L_{100} \\
L_{250}, L_{500}, L_{750}, L_{1000}, L_{1250}, L_{1500}, L_{\infty}\end{array}$ & $\begin{array}{l}L_{K}> \\
L_{K}= \\
L_{K}<\end{array}$ & $\begin{array}{l}L_{0} *, L_{100} \\
L_{250}, L_{500}, L_{750}, L_{1000}, L_{1250}, L_{1500}, L_{\infty}\end{array}$ \\
\hline $\mathrm{D}=\mathbf{5 0}$ & $\begin{array}{l}L_{K}> \\
L_{K}= \\
L_{K}<\end{array}$ & $\begin{array}{l}L_{0} *, L_{100} *, L_{250} *, L_{500} *, L_{750} *, L_{1000} * \\
L_{1250} *, L_{1500} *, L_{\infty} *\end{array}$ & $\begin{array}{l}L_{K}> \\
L_{K}= \\
L_{K}<\end{array}$ & $\begin{array}{l}L_{0} *, L_{100} \\
L_{250}, L_{500}, L_{750}, L_{1000}, L_{1250}, L_{1500}, L_{\infty}\end{array}$ & $\begin{array}{l}L_{K}> \\
L_{K}= \\
L_{K}<\end{array}$ & $\begin{array}{l}L_{0} *, L_{100} *, L_{250} *, L_{500} *, L_{750} *, L_{1000} * \\
L_{1250} *, L_{1500} *, L_{\infty} *\end{array}$ \\
\hline
\end{tabular}

Table 41

$f_{5}$, vertical approach, MaxFEs $=250,000$, CRS4EAs

\begin{tabular}{|c|c|c|c|c|c|c|}
\hline & & $\mathrm{SN}=24$ & & $\mathrm{SN}=50$ & & $\mathrm{SN}=100$ \\
\hline $\mathbf{D}=\mathbf{2}$ & $\begin{array}{l}L_{K}> \\
L_{K}= \\
L_{K}<\end{array}$ & $\begin{array}{l}L_{0} *, L_{100} *, L_{250} *, L_{500} *, L_{750} *, L_{1000} * \\
L_{1250} *, L_{1500} *, L_{\infty} *\end{array}$ & $\begin{array}{l}L_{K}> \\
L_{K}= \\
L_{K}<\end{array}$ & $\begin{array}{l}L_{0} *, L_{100} *, L_{250} *, L_{500} *, L_{750} *, L_{1000} * \\
L_{1250} *, L_{1500} *, L_{\infty} *\end{array}$ & $\begin{array}{l}L_{K}> \\
L_{K}= \\
L_{K}<\end{array}$ & $\begin{array}{l}L_{0}, L_{250} *, L_{500} *, L_{750} *, L_{1000} *, L_{1250} * \\
L_{1500} *, L_{\infty} * \\
L_{100}\end{array}$ \\
\hline$D=10$ & $\begin{array}{l}L_{K}> \\
L_{K}= \\
L_{K}<\end{array}$ & $L_{0} *, L_{100} *, L_{250}, L_{500}$ & $\begin{array}{l}L_{K}> \\
L_{K}= \\
L_{K}<\end{array}$ & $\begin{array}{l}L_{0} *, L_{100} *, L_{500}, L_{750}, L_{1500}, \\
L_{250} \\
L_{1000}, L_{1250}, L_{\infty}\end{array}$ & $\begin{array}{l}L_{K}> \\
L_{K}= \\
L_{K}<\end{array}$ & $\begin{array}{l}L_{0} *, L_{100} *, L_{250}, L_{750}, L_{1000}, L_{1250}, \\
L_{1500}, L_{\infty} \\
L_{500}\end{array}$ \\
\hline $\mathbf{D}=30$ & $\begin{array}{l}L_{K}> \\
L_{K}= \\
L_{K}<\end{array}$ & $\begin{array}{l}L_{0} *, L_{100} *, L_{250} *, L_{500} *, L_{750} *, L_{1000} * \\
L_{1250} *, L_{1500} *, L_{\infty} *\end{array}$ & $\begin{array}{l}L_{K}> \\
L_{K}= \\
L_{K}<\end{array}$ & $\begin{array}{l}L_{0} *, L_{100} *, L_{250} *, L_{500} *, L_{1000} *, L_{1250} * \\
L_{1500} *, L_{\infty} * \\
L_{750}\end{array}$ & $\begin{array}{l}L_{K}> \\
L_{K}= \\
L_{K}<\end{array}$ & $\begin{array}{l}L_{0} *, L_{100} *, L_{250} *, L_{500} *, L_{750} *, L_{1000} * \\
L_{1250} *, L_{\infty} * \\
L_{1500}\end{array}$ \\
\hline $\mathrm{D}=\mathbf{5 0}$ & $\begin{array}{l}L_{K}> \\
L_{K}= \\
L_{K}<\end{array}$ & $\begin{array}{l}L_{0} *, L_{100} *, L_{250} *, L_{500} *, L_{750} *, L_{1000} * \\
L_{1250} *, L_{1500} *, L_{\infty} *\end{array}$ & $\begin{array}{l}L_{K}> \\
L_{K}= \\
L_{K}<\end{array}$ & $\begin{array}{l}L_{0} *, L_{100} *, L_{250} *, L_{500} *, L_{750} *, L_{1000} * \\
L_{1500} *, L_{\infty} * \\
L_{1250}\end{array}$ & $\begin{array}{l}L_{K}> \\
L_{K}= \\
L_{K}<\end{array}$ & $\begin{array}{l}L_{0} *, L_{100} *, L_{250} *, L_{500} *, L_{750} *, L_{1000} * \\
L_{1250}, L_{1500} *, L_{\infty} *\end{array}$ \\
\hline
\end{tabular}


Table 42

Horizontal approach, $10^{-6}$

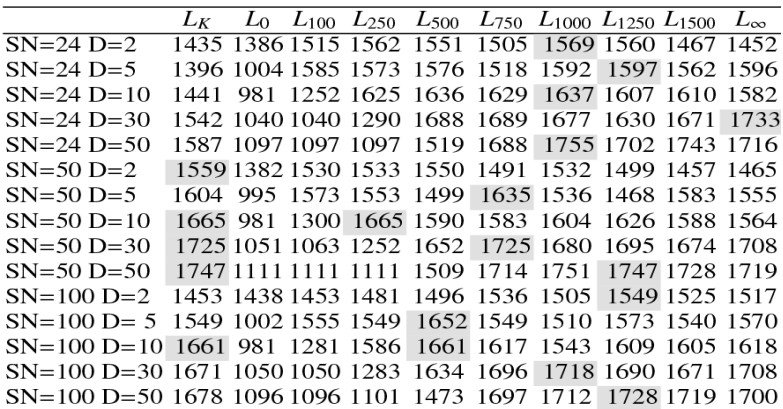
(a) Horizontal approach, $f_{1}, 10^{-6}$

$\begin{array}{lllllllllll} & L_{K} & L_{0} & L_{100} & L_{250} & L_{500} & L_{750} & L_{1000} & L_{1250} & L_{1500} & L_{\infty}\end{array}$ $\begin{array}{llllllllllll}1553 & 1082 & 1566 & 1521 & 1578 & 1559 & 1537 & 1544 & 1565 & 1495\end{array}$ $\mathrm{SN}=24 \mathrm{D}=5 \quad 1553 \quad 981 \quad 1576 \quad 1581 \quad 1571 \quad 1516 \quad 1548 \quad 1576 \quad 1553 \quad 1546$

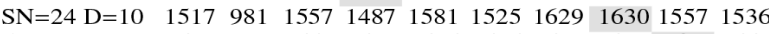

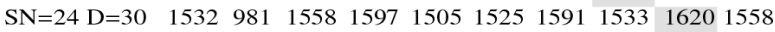

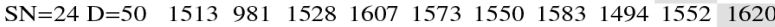
$\begin{array}{lllllllllllll}\mathrm{SN}=50 \quad \mathrm{D}=2 & 1560 & 1058 & 1624 & 1589 & 1557 & 1555 & 1500 & 1501 & 1535 & 1521\end{array}$ $\begin{array}{llllllllllll}\mathrm{SN}=50 & \mathrm{D}=5 & 1538 & 981 & 1577 & 1591 & 1595 & 1541 & 1513 & 1558 & 1559 & 1547\end{array}$ \begin{tabular}{l|lllllllllll}
$\mathrm{SN}=50$ & $\mathrm{D}=10$ & 1618 & 981 & 1579 & 1618 & 1532 & 1574 & 1583 & 1547 & 1519 & 1566
\end{tabular} $\begin{array}{llllllllllll}\mathrm{SN}=50 & \mathrm{D}=30 & 1603 & 981 & 1510 & 1582 & 1544 & 1603 & 1564 & 1592 & 1528 & 1595\end{array}$ $\begin{array}{lllllllllllll}\mathrm{SN}=50 & \mathrm{D}=50 & 1579 & 981 & 1565 & 1516 & 1579 & 1569 & 1578 & 1579 & 1569 & 1565\end{array}$

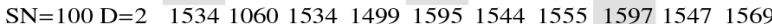
$\begin{array}{lllllllllll}\mathrm{SN}=100 \mathrm{D}=5 & 1592 & 981 & 1571 & 1592 & 1519 & 1608 & 1546 & 1521 & 1569 & 1593\end{array}$

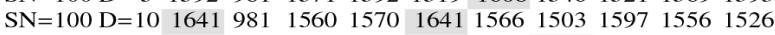

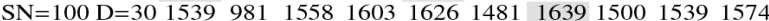

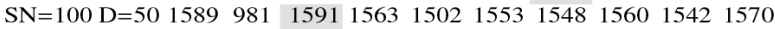

(b) Horizontal approach, $f_{2}, 10^{-6}$

\begin{tabular}{lllllllllll}
\hline$L_{K}$ & $L_{0}$ & $L_{100}$ & $L_{250}$ & $L_{500}$ & $L_{750}$ & $L_{1000}$ & $L_{1250}$ & $L_{1500}$ & $L_{\infty}$
\end{tabular}

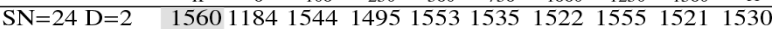

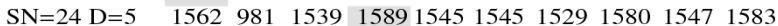

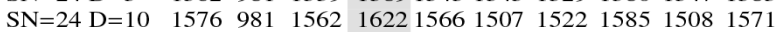

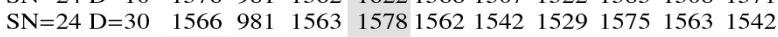

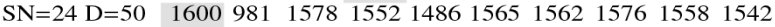

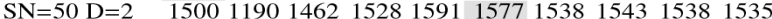
$\mathrm{SN}=50 \mathrm{D}=5 \quad 1586 \quad 981 \quad 1548 \quad 1561 \quad 1574 \quad 1535 \quad 15591556 \quad 1497 \quad 1605$

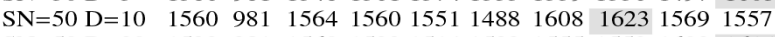
$\begin{array}{lllllllllllll}\mathrm{SN}=50 & \mathrm{D}=30 & 1529 & 981 & 1560 & 1592 & 1514 & 1529 & 1555 & 1553 & 1603 & 1614\end{array}$

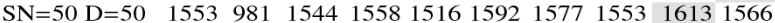
$\mathrm{SN}=100 \mathrm{D}=2 \quad 150111691501 \quad 15231508 \quad 1575 \quad 1596152015951513$ $\begin{array}{llllllllllll}\mathrm{SN}=100 & \mathrm{D}=5 & 1578 & 981 & 1613 & 1578 & 1603 & 1590 & 1571 & 1527 & 1514 & 1523\end{array}$

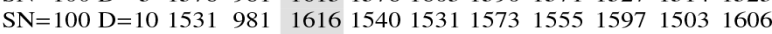

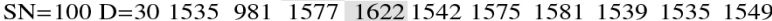
SN=100 D=50 $1553 \quad 981 \quad 1559 \quad 1524 \quad 1572 \quad 1544 \quad 1514 \quad 1603 \quad 1591 \quad 1560$ (c) Horizontal approach, $f_{3}, 10^{-6}$

\begin{tabular}{lcccccccccc}
\hline & $L_{K}$ & $L_{0}$ & $L_{100}$ & $L_{250}$ & $L_{500}$ & $L_{750}$ & $L_{1000}$ & $L_{1250}$ & $L_{1500}$ & $L_{\infty}$ \\
\hline $\mathrm{SN}=24 \mathrm{D}=2$ & 1411 & 1115 & 1588 & 1573 & 1528 & 1557 & 1534 & 1550 & 1518 & 1628 \\
$\mathrm{SN}=24 \mathrm{D}=5$ & 1562 & 981 & 1533 & 1549 & 1546 & 1572 & 1629 & 1583 & 1516 & 1528 \\
$\mathrm{SN}=24 \mathrm{D}=10$ & 1576 & 981 & 1536 & 1521 & 1578 & 1590 & 1531 & 1618 & 1518 & 1551 \\
$\mathrm{SN}=24 \mathrm{D}=30$ & 1527 & 981 & 1598 & 1593 & 1554 & 1499 & 1593 & 1561 & 1585 & 1508 \\
$\mathrm{SN}=24 \mathrm{D}=50$ & 1582 & 981 & 1575 & 1544 & 1535 & 1538 & 1523 & 1544 & 1617 & 1562 \\
$\mathrm{SN}=50 \mathrm{D}=2$ & 1527 & 1100 & 1548 & 1538 & 1542 & 1543 & 1494 & 1553 & 1602 & 1555 \\
$\mathrm{SN}=50 \mathrm{D}=5$ & 1573 & 981 & 1540 & 1502 & 1597 & 1583 & 1544 & 1554 & 1560 & 1566 \\
$\mathrm{SN}=50 \mathrm{D}=10$ & 1552 & 981 & 1597 & 1552 & 1614 & 1587 & 1532 & 1551 & 1552 & 1535 \\
$\mathrm{SN}=50 \mathrm{D}=30$ & 1578 & 981 & 1593 & 1531 & 1565 & 1578 & 1513 & 1590 & 1558 & 1591 \\
$\mathrm{SN}=50 \mathrm{D}=50$ & 1535 & 981 & 1605 & 1622 & 1565 & 1565 & 1561 & 1560 & 1535 & 1506 \\
$\mathrm{SN}=100 \mathrm{D}=2$ & 1575 & 1074 & 1575 & 1529 & 1597 & 1523 & 1578 & 1556 & 1553 & 1516 \\
$\mathrm{SN}=100 \mathrm{D}=5$ & 1574 & 981 & 1551 & 1574 & 1551 & 1559 & 1535 & 1544 & 1612 & 1593 \\
$\mathrm{SN}=100 \mathrm{D}=10$ & 1590 & 981 & 1583 & 1549 & 1590 & 1557 & 1570 & 1592 & 1540 & 1538 \\
$\mathrm{SN}=100 \mathrm{D}=30$ & 1586 & 981 & 1575 & 1535 & 1586 & 1547 & 1556 & 1599 & 1586 & 1536 \\
$\mathrm{SN}=100 \mathrm{D}=50$ & 1567 & 981 & 1566 & 1510 & 1521 & 1597 & 1567 & 1554 & 1561 & 1576 \\
\hline
\end{tabular} (d) Horizontal approach, $f_{4}, 10^{-6}$

\begin{tabular}{lcccccccccc}
\hline & $L_{K}$ & $L_{0}$ & $L_{100}$ & $L_{250}$ & $L_{500}$ & $L_{750}$ & $L_{1000}$ & $L_{1250}$ & $L_{1500}$ & $L_{\infty}$ \\
\hline $\mathrm{SN}=24 \mathrm{D}=2$ & 1499 & 1499 & 1499 & 1499 & 1499 & 1499 & 1499 & 1499 & 1499 & 1510 \\
$\mathrm{SN}=24 \mathrm{D}=5$ & 1500 & 1500 & 1500 & 1500 & 1500 & 1500 & 1500 & 1500 & 1500 & 1500 \\
$\mathrm{SN}=24 \mathrm{D}=10$ & 1500 & 1500 & 1500 & 1500 & 1500 & 1500 & 1500 & 1500 & 1500 & 1500 \\
$\mathrm{SN}=24 \mathrm{D}=30$ & 1500 & 1500 & 1500 & 1500 & 1500 & 1500 & 1500 & 1500 & 1500 & 1500 \\
$\mathrm{SN}=24 \mathrm{D}=50$ & 1500 & 1500 & 1500 & 1500 & 1500 & 1500 & 1500 & 1500 & 1500 & 1500 \\
$\mathrm{SN}=50 \mathrm{D}=2$ & 1498 & 1498 & 1498 & 1503 & 1498 & 1503 & 1503 & 1498 & 1498 & 1503 \\
$\mathrm{SN}=50 \mathrm{D}=5$ & 1500 & 1500 & 1500 & 1500 & 1500 & 1500 & 1500 & 1500 & 1500 & 1500 \\
$\mathrm{SN}=50 \mathrm{D}=10$ & 1500 & 1500 & 1500 & 1500 & 1500 & 1500 & 1500 & 1500 & 1500 & 1500 \\
$\mathrm{SN}=50 \mathrm{D}=30$ & 1500 & 1500 & 1500 & 1500 & 1500 & 1500 & 1500 & 1500 & 1500 & 1500 \\
$\mathrm{SN}=50 \mathrm{D}=50$ & 1500 & 1500 & 1500 & 1500 & 1500 & 1500 & 1500 & 1500 & 1500 & 1500 \\
$\mathrm{SN}=100 \mathrm{D}=2$ & 1499 & 1505 & 1499 & 1499 & 1499 & 1499 & 1505 & 1499 & 1499 & 1499 \\
$\mathrm{SN}=100 \mathrm{D}=5$ & 1500 & 1500 & 1500 & 1500 & 1500 & 1500 & 1500 & 1500 & 1500 & 1500 \\
$\mathrm{SN}=100 \mathrm{D}=10$ & 1500 & 1500 & 1500 & 1500 & 1500 & 1500 & 1500 & 1500 & 1500 & 1500 \\
$\mathrm{SN}=100 \mathrm{D}=30$ & 1500 & 1500 & 1500 & 1500 & 1500 & 1500 & 1500 & 1500 & 1500 & 1500 \\
$\mathrm{SN}=100 \mathrm{D}=50$ & 1500 & 1500 & 1500 & 1500 & 1500 & 1500 & 1500 & 1500 & 1500 & 1500 \\
\hline
\end{tabular}

(e) Horizontal approach, $f_{5}, 10^{-6}$

least one other 'limit' value, regardless of the population size and dimension of a problem. Lastly, for $f_{5}$ and small dimension $D=2, L_{k}$ had better alternatives, whilst for other dimensions and population sizes values, all 'limit' values performed the same. This happened due to the fact that none of these 'limit' values found the (sub-)optimal solution $10^{-6}$ in 1,000,000 available fitness evaluations. For $D=2$, some 'limit' values found (sub-)optimal solution in some runs, and therefore performed better than $L_{k}$. Whilst there were a lot of differences found between $L_{k}$ and other 'limit' values, these differences were hardly ever significant. There were only two problems for which $L_{k}$ was significantly worse than some other 'limit' values. The first was $f_{1}$ where $L_{k}$ was significantly worse for small population size $S N=24$ and dimension $D=5$. The other problem was $f_{4}$ where $L_{k}$ was significantly worse than $L_{\infty}$ for small population size $S N=24$ and small dimension $D=2$. CRS4EAs again appeared as more conservative than NHST (compare Tables 15-19 with Tables 43-47).

\subsubsection{Experiment 4: Horizontal Approach $-10^{-12}-$ MaxFEs $=1,000,000$}

In the horizontal approach with (sub-)optimal solution $10^{-12}, L_{k}$ again had better alternatives in almost all cases. In this approach, $L_{k}$ was the optimal solution $50 \%$ fewer times than when the (sub-)optimal solution equaled $10^{-6}$. Tables $48(\mathrm{a})-48(\mathrm{e})$ show the ratings obtained for all five optimisation problems.

For $f_{1}, L_{k}$ always had a better alternative, except when $S N=50$ and $D=10, S N=50$ and $D=30$, and $S N=100$ and $D=2$. For $f_{2}, L_{k}$ always had a better alternative and was always worse than at least one other 'limit' value, regardless of the population size and dimension of a problem. For $f_{3}, L_{k}$ always had a better alternative and 
was always worse than at least one other 'limit' value, except when $S N=50$ and $D=5$. For $f_{4}, L_{k}$ always had a better alternative and was always worse than at least one other 'limit' value, except when $S N=24$ and $D=30$ and $S N=100$ and $D=30$. Lastly, for $f_{5}$, all 'limit' values performed the same. This happened due to the fact that none of these 'limit' values found the (sub-)optimal solution $10^{-12}$ in 1,000,000 fitness evaluations. Whilst there were a lot of differences found between $L_{k}$ and other 'limit' values, these differences were rarely significant. There were only two problems for which $L_{k}$ was significantly worse than some other 'limit' values. The first was $f_{1}$ where $L_{k}$ was significantly worse for small population size $S N=24$ and dimensions $D=\{5,10,30,50\}$. The other was problem $f_{4}$ where $L_{k}$ was significantly worse for small population size $S N=24$ and small dimension $D=$ 2. CRS4EAs again appeared as more conservative than NHST (compare Tables 20-24 with Tables 49-53).

\subsubsection{Experiment 5: Large Dimensions}

In this section, the horizontal approach with (sub-) optimal solution set at $10^{-6}$ was repeated for larger dimensions, $D=\{100,200,300\}$. Again, fixed 'limit' values, $L=\{0,1000,2000,3000,4000,5000,6000$, $7000,8000,9000,10000,11000,12000,13000,14000$, $15000, \infty$, were compared to Karaboga's setting $L_{k}$. Obtained ratings are shown in Table 54 and found differences are shown in Tables 55-59. As in previous four experiments, this experiment showed that there are other 'limit' values that perform better than $L_{k}$, for certain problems $\left(f_{1}\right)$ even significantly. In majority of $D$ and $S N$ settings and problems, at least one better performing 'limit' value was found. For $f_{5}$ none of the 'limit' values reached optimal solution, since all settings performed equally. By comparing Tables 55-59 with Tables 43-47, it can be observed that with higher dimensions $L_{k}$ setting becomes less appropriate.

Table 43

$f_{1}$, horizontal approach, $10^{-6}$, CRS4EAs

\begin{tabular}{|c|c|c|c|c|c|c|}
\hline & & $\mathrm{SN}=24$ & & $\mathrm{SN}=\mathbf{5 0}$ & & $\mathrm{SN}=100$ \\
\hline \multirow{3}{*}{$D=2$} & $L_{K}>$ & $L_{0}$ & $L_{K}>$ & $\begin{array}{l}L_{0}, L_{100}, L_{250}, L_{500}, L_{750}, L_{1000}, L_{1250}, \\
L_{1500}, L_{\infty}\end{array}$ & $L_{K}>$ & $L_{0}$ \\
\hline & $L_{K}=$ & & $L_{K}=$ & & $L_{K}=$ & $L_{100}$ \\
\hline & $L_{K}<$ & $\begin{array}{l}L_{100}, L_{250}, L_{500}, L_{750}, L_{1000}, L_{1250}, \\
L_{1500}, L_{\infty}\end{array}$ & $L_{K}<$ & & $L_{K}<$ & $L_{250}, L_{500}, L_{750}, L_{1000}, L_{1250}, L_{1500}, L_{\infty}$ \\
\hline \multirow{3}{*}{$\mathrm{D}=5$} & $L_{K}>$ & $L_{0} *$ & & $\begin{array}{l}L_{0} *, L_{100}, L_{250}, L_{500}, L_{1000}, L_{1250}, \\
L_{1500}, L_{\infty}\end{array}$ & $L_{K}>$ & $L_{0} *, L_{1000}, L_{1500}$ \\
\hline & $L_{K}=$ & & $L_{K}=$ & & $L_{K}=$ & $L_{250}, L_{750}$ \\
\hline & $L_{K}<$ & $\begin{array}{l}L_{100}, L_{250}, L_{500}, L_{750}, L_{1000}, L_{1250} * \text {, } \\
L_{1500}, L_{\infty}\end{array}$ & $L_{K}<$ & $L_{750}$ & $L_{K}<$ & $L_{100}, L_{500}, L_{1250}, L_{\infty}$ \\
\hline \multirow[b]{2}{*}{$D=10$} & $L_{K}>$ & $L_{0} *, L_{100}$ & & $\begin{array}{l}L_{0} *, L_{100} *, L_{500}, L_{750}, L_{1000}, L_{1250}, \\
L_{1500}, L_{\infty}\end{array}$ & & $\begin{array}{l}L_{0} *, L_{100} *, L_{250}, L_{750}, L_{1000}, L_{1250}, \\
L_{1500}, L_{\infty}\end{array}$ \\
\hline & $\begin{array}{l}L_{K}= \\
L_{K}<\end{array}$ & $L_{250}, L_{500}, L_{750}, L_{1000}, L_{1250}, L_{1500}, L_{\infty}$ & $\begin{array}{l}L_{K}= \\
L_{K}<\end{array}$ & $L_{250}$ & $\begin{array}{l}L_{K}= \\
L_{K}<\end{array}$ & $L_{500}$ \\
\hline \multirow[b]{2}{*}{$\mathrm{D}=\mathbf{3 0}$} & $L_{K}>$ & $L_{0} *, L_{100} *, L_{250} *$ & $L_{K}>$ & $\begin{array}{l}L_{0} *, L_{100} *, L_{250} *, L_{500}, L_{1000}, L_{1250}, \\
L_{1500}, L_{\infty}\end{array}$ & $L_{K}>$ & $L_{0} *, L_{100} *, L_{250} *, L_{500}$ \\
\hline & $L_{K}=$ & $L_{500} L_{750} L_{1000} L_{1250} L_{1500}, L_{0}$ & $L_{K}=$ & $L_{750}$ & $L_{K}=$ & $L_{1500}$ \\
\hline \multirow[b]{2}{*}{$\mathrm{D}=\mathbf{5 0}$} & $\frac{L_{K}<}{L_{K}>}$ & $L_{0} *, L_{100} *, L_{250} *, L_{500}$ & $L_{K}>$ & $\begin{array}{l}L_{0} *, L_{100} *, L_{250} *, L_{500} *, L_{750}, L_{1500}, \\
L_{\infty}\end{array}$ & $\frac{L_{K}<}{L_{K}>}$ & $L_{0} *, L_{100} *, L_{250} *, L_{500} *$ \\
\hline & $\begin{array}{l}L_{K}= \\
L_{K}<\end{array}$ & $L_{750}, L_{1000}, L_{1250}, L_{1500}, L_{\infty}$ & $\begin{array}{l}L_{K}= \\
L_{K}<\end{array}$ & $\begin{array}{l}L_{1250} \\
L_{1000}\end{array}$ & $\begin{array}{l}L_{K}= \\
L_{K}<\end{array}$ & $L_{750}, L_{1000}, L_{1250}, L_{1500}, L_{\infty}$ \\
\hline
\end{tabular}

\section{Table 44}

$f_{2}$, horizontal approach, $10^{-6}$, CRS4EAs

\begin{tabular}{|c|c|c|c|c|c|c|}
\hline & & $\mathrm{SN}=24$ & & $\mathrm{SN}=50$ & & $\mathrm{SN}=100$ \\
\hline$D=2$ & $\begin{array}{l}L_{K}> \\
L_{K}=\end{array}$ & $L_{0} *, L_{250}, L_{1000}, L_{1250}, L_{\infty}$ & $\begin{array}{l}L_{K}> \\
L_{K}=\end{array}$ & $L_{0} *, L_{500}, L_{750}, L_{1000}, L_{1250}, L_{1500}, L_{\infty}$ & $\begin{array}{l}L_{K}> \\
L_{K}=\end{array}$ & $\begin{array}{l}L_{0} *, L_{250}, \\
L_{100}\end{array}$ \\
\hline & $L_{K}<$ & $L_{100}, L_{500}, L_{750}, L_{1500}$ & $L_{K}<$ & $L_{100}, L_{250}$ & $L_{K}<$ & $L_{500}, L_{750}, L_{1000}, L_{1250}, L_{1500}, L_{\infty}$ \\
\hline$D=5$ & $\begin{array}{l}L_{K}> \\
L_{K}= \\
L_{K}<\end{array}$ & $\begin{array}{l}L_{0} *, L_{750}, L_{1000}, L_{\infty} \\
L_{1500} \\
L_{100}, L_{250}, L_{500}, L_{1250}\end{array}$ & $\begin{array}{l}L_{K}> \\
L_{K}= \\
L_{K}<\end{array}$ & $\begin{array}{l}L_{0} *, L_{1000} \\
L_{100}, L_{250}, L_{500}, L_{750}, L_{1250}, L_{1500}, L_{\infty}\end{array}$ & $\begin{array}{l}L_{K}> \\
L_{K}= \\
L_{K}<\end{array}$ & $\begin{array}{l}L_{0}, L_{100}, L_{500}, L_{1000}, L_{1250}, L_{1500} \\
L_{250} \\
L_{750}, L_{\infty}\end{array}$ \\
\hline $\mathbf{D}=\mathbf{1 0}$ & $\begin{array}{l}L_{K}= \\
L_{K}<\end{array}$ & $L_{100}, L_{500}, L_{750}, L_{1000}, L_{1250}, L_{1500}, L_{\infty}$ & $\begin{array}{l}L_{K}> \\
L_{K}= \\
L_{K}<\end{array}$ & $\begin{array}{l}L_{0} *, L_{100}, L_{500}, L_{750}, L_{1000}, L_{1250} \\
L_{1500}, L_{\infty} \\
L_{250}\end{array}$ & $\begin{array}{l}L_{K}> \\
L_{K}= \\
L_{K}<\end{array}$ & $\begin{array}{l}L_{0}, L_{100}, L_{250}, L_{750}, L_{1000}, L_{1250}, L_{1500}, \\
L_{\infty} \\
L_{500}\end{array}$ \\
\hline $\mathbf{D}=\mathbf{3 0}$ & $\begin{array}{l}L_{K}= \\
L_{K}<\end{array}$ & $L_{100}, L_{250}, L_{1000}, L_{1250}, L_{1500}, L_{\infty}$ & $\begin{array}{l}L_{K}= \\
L_{K}<\end{array}$ & $\begin{array}{l}L_{0} *, L_{100}, L_{250}, L_{500}, L_{1000}, L_{1250} \\
L_{1500}, L_{\infty} \\
L_{750}\end{array}$ & $\begin{array}{l}L_{K}> \\
L_{K}= \\
L_{K}<\end{array}$ & $\begin{array}{l}L_{1500} \\
L_{100}, L_{250}, L_{500}, L_{1000}, L_{\infty}\end{array}$ \\
\hline$D=50$ & $\begin{array}{l}L_{K}= \\
L_{K}<\end{array}$ & $L_{100}, L_{250}, L_{500}, L_{750}, L_{1000}, L_{1500}, L_{\infty}$ & $\begin{array}{l}L_{K}= \\
L_{K}<\end{array}$ & $L_{0} *, L_{100}, L_{250}, L_{750}, L_{1000}, L_{1500}, L_{\infty}$ & $\begin{array}{l}L_{K}= \\
L_{K}<\end{array}$ & $\begin{array}{l}L_{0}, L_{250}, L_{500}, L_{750}, L_{1000}, L_{1250}, L_{1500}, \\
L_{\infty} \\
L_{100}\end{array}$ \\
\hline
\end{tabular}


Table 45

$f_{3}$, horizontal approach, $10^{-6}$, CRS4EAs

\begin{tabular}{|c|c|c|c|c|c|c|}
\hline & & $\mathrm{SN}=24$ & & $\mathrm{SN}=\mathbf{5 0}$ & & $\mathrm{SN}=100$ \\
\hline & $L_{K}>$ & $L_{0} *, L_{100}, L_{250}, L_{500}, L_{750}, L_{1000}, L_{1250}$ & $L_{K}>$ & $L_{0} *, L_{100} *$ & $L_{K}>$ & $L_{0} *$ \\
\hline \multirow[t]{2}{*}{$\mathbf{D}=2$} & $L_{K}=$ & & $L_{K}=$ & & $L_{K}=$ & $L_{100}$ \\
\hline & $L_{K}<$ & & $L_{K}<$ & $L_{250}, L_{500}, L_{750}, L_{1000}, L_{1250}, L_{1500}, L_{\infty}$ & $L_{K}<$ & $L_{250}, L_{500}, L_{750}, L_{1000}, L_{1250}, L_{1500}, L_{\infty}$ \\
\hline \multirow[t]{2}{*}{$D=5$} & $L_{K}=$ & & $L_{K}=$ & & $L_{K}=$ & $L_{250}$ \\
\hline & $L_{K}<$ & $L_{250}, L_{1250}, L_{\infty}$ & $L_{K}^{\mathrm{K}}<$ & $L_{\infty}$ & $L_{K}<$ & $L_{100}, L_{500}, L_{750}$ \\
\hline \multirow[t]{2}{*}{$D=10$} & $\begin{array}{l}L_{K}> \\
L_{K}=\end{array}$ & $L_{0} *, L_{100}, L_{500}, L_{750}, L_{1000}, L_{1500}, L_{\infty}$ & $\begin{array}{l}L_{K}> \\
L_{K}=\end{array}$ & $\begin{array}{l}L_{0} *, L_{500}, L_{750}, L_{\infty} \\
L_{250}\end{array}$ & $\begin{array}{l}L_{K}> \\
L_{K}=\end{array}$ & $\begin{array}{l}L_{0} *, L_{1500} \\
L_{500}\end{array}$ \\
\hline & $L_{K}<$ & $L_{250}, L_{1250}$ & $L_{K}<$ & $L_{100}, L_{1000}, L_{1250}, L_{1500}$ & $L_{K}<$ & $L_{100}, L_{250}, L_{750}, L_{1000}, L_{1250}, L_{\infty}$ \\
\hline \multirow[t]{2}{*}{$\mathbf{D}=\mathbf{3 0}$} & $\begin{array}{l}L_{K}> \\
L_{K}=\end{array}$ & $L_{0} *, L_{100}, L_{500}, L_{750}, L_{1000}, L_{1500}, L_{\infty}$ & $\begin{array}{l}L_{K}> \\
L_{K}=\end{array}$ & $\begin{array}{l}L_{0} *, L_{500} \\
L_{750}\end{array}$ & $\begin{array}{l}L_{K}> \\
L_{K}=\end{array}$ & $\begin{array}{l}L_{0} * \\
L_{1500}\end{array}$ \\
\hline & $L_{K}<$ & $L_{250}, L_{1250}$ & $L_{K}<$ & $L_{100}, L_{250}, L_{1000}, L_{1250}, L_{1500}, L_{\infty}$ & $L_{K}<$ & $L_{100}, L_{250}, L_{500}, L_{750}, L_{1000}, L_{1250}, L_{\infty}$ \\
\hline & $L_{K}>$ & $\begin{array}{l}L_{0} *, L_{100}, L_{250}, L_{500}, L_{750}, L_{1000}, L_{1250}, \\
L_{1500}, L_{\infty}\end{array}$ & $L_{K}>$ & $L_{0} *, L_{100}, L_{500}$ & $L_{K}>$ & $L_{0} *, L_{250}, L_{750}, L_{1000}$ \\
\hline $\mathbf{D}=\mathbf{5 0}$ & $\begin{array}{l}L_{K}= \\
L_{K}<\end{array}$ & & $\begin{array}{l}L_{K}= \\
L_{K}<\end{array}$ & $\begin{array}{l}L_{1250} \\
L_{250}, L_{750}, L_{1000}, L_{1500}, L_{\infty}\end{array}$ & $\begin{array}{l}L_{K}= \\
L_{K}<\end{array}$ & $L_{100}, L_{500}, L_{1250}, L_{1500}, L_{\infty}$ \\
\hline
\end{tabular}

\section{Table 46}

$f_{4}$, horizontal approach, $10^{-6}$, CRS4EAs

\begin{tabular}{|c|c|c|c|c|c|c|}
\hline & & $\mathrm{SN}=24$ & & $\mathrm{SN}=\mathbf{5 0}$ & & $\mathrm{SN}=100$ \\
\hline \multirow[t]{2}{*}{$\mathbf{D}=2$} & $\begin{array}{l}L_{K}> \\
L_{K}=\end{array}$ & $L_{0} *$ & $\begin{array}{l}L_{K}> \\
L_{K}=\end{array}$ & $L_{0} *, L_{1000}$ & $\begin{array}{l}L_{K}> \\
L_{K}=\end{array}$ & $\begin{array}{l}L_{0} *, L_{250}, L_{750}, L_{1250}, L_{1500}, L_{\infty} \\
L_{100}\end{array}$ \\
\hline & $L_{K}<$ & $\begin{array}{l}L_{100}, L_{250}, L_{500}, L_{750}, L_{1000}, L_{1250}, \\
L_{1500}, L_{\infty} *\end{array}$ & $L_{K}^{\Lambda}<$ & $L_{100}, L_{250}, L_{500}, L_{750}, L_{1250}, L_{1500}, L_{\infty}$ & $L_{K}^{\Lambda}<$ & $L_{500}, L_{1000}$ \\
\hline \multirow[t]{2}{*}{$\mathbf{D}=\mathbf{5}$} & $\begin{array}{l}L_{K}> \\
L_{K}=\end{array}$ & $L_{0} *, L_{100}, L_{250}, L_{500}, L_{1500}, L_{\infty}$ & $\begin{array}{l}L_{K}> \\
L_{K}=\end{array}$ & $L_{0} *, L_{100}, L_{250}, L_{1000}, L_{1250}, L_{1500}, L_{\infty}$ & $\begin{array}{l}L_{K}> \\
L_{K}=\end{array}$ & $\begin{array}{l}L_{0} *, L_{100}, L_{500}, L_{750}, L_{1000}, L_{1250} \\
L_{250}\end{array}$ \\
\hline & $L_{K}^{n}<$ & $L_{750}, L_{1000}, L_{1250}$ & $L_{K}<$ & $L_{500}, L_{750}$, & $L_{K}<$ & $L_{1500}, L_{\infty}$ \\
\hline$D=10$ & $\begin{array}{l}L_{K}> \\
L_{K}= \\
L_{K}<\end{array}$ & $\begin{array}{l}L_{0} *, L_{100}, L_{250}, L_{1000}, L_{1500}, L_{\infty} \\
L_{500}, L_{750}, L_{1250}\end{array}$ & $\begin{array}{l}L_{K}> \\
L_{K}= \\
L_{K}<\end{array}$ & $\begin{array}{l}L_{0} *, L_{1000}, L_{1250}, L_{\infty} \\
L_{250}, L_{1500} \\
L_{100}, L_{500}, L_{750}\end{array}$ & $\begin{array}{l}L_{K}> \\
L_{K}= \\
L_{K}<\end{array}$ & $\begin{array}{l}L_{0} *, L_{100}, L_{250}, L_{750}, L_{1000}, L_{1500}, L_{\infty} \\
L_{500} \\
L_{1250}\end{array}$ \\
\hline $\mathbf{D}=\mathbf{3 0}$ & $\begin{array}{l}L_{K}> \\
L_{K}= \\
L_{K}<\end{array}$ & $\begin{array}{l}L_{0} *, L_{750}, L_{\infty} \\
L_{100}, L_{250}, L_{500}, L_{1000}, L_{1250}, L_{1500}\end{array}$ & $\begin{array}{l}L_{K}> \\
L_{K}= \\
L_{K}<\end{array}$ & $\begin{array}{l}L_{0} *, L_{250}, L_{500}, L_{1000}, L_{1500} \\
L_{750} \\
L_{100}, L_{1250}, L_{\infty}\end{array}$ & $\begin{array}{l}L_{K}> \\
L_{K}= \\
L_{K}<\end{array}$ & $\begin{array}{l}L_{0} *, L_{100}, L_{250}, L_{750}, L_{1000}, L_{\infty} \\
L_{500}, L_{1500} \\
L_{1250}\end{array}$ \\
\hline \multirow{3}{*}{$\mathbf{D}=\mathbf{5 0}$} & $L_{K}>$ & $\begin{array}{l}L_{0} *, L_{100}, L_{250}, L_{500}, L_{750}, L_{1000}, L_{1250}, \\
L_{\infty}\end{array}$ & $L_{K}>$ & $L_{0} *, L_{1500}, L_{\infty}$ & $L_{K}>$ & $L_{0} *, L_{100}, L_{250}, L_{500}, L_{1250}, L_{1500}$ \\
\hline & $L_{K}=$ & & $L_{K}=$ & & $L_{K}=$ & $L_{1000}$ \\
\hline & $L_{K}<$ & $L_{1500}$ & $L_{K}<$ & $L_{100}, L_{250}, L_{500}, L_{750}, L_{1000}, L_{1250}$ & $L_{K}<$ & $L_{750}, L_{\infty}$ \\
\hline
\end{tabular}

\section{Table 47}

$f_{5}$, horizontal approach, $10^{-6}$, CRS4EAs

\begin{tabular}{|c|c|c|c|c|c|c|}
\hline & & $\mathrm{SN}=24$ & & $\mathrm{SN}=50$ & & $\mathrm{SN}=100$ \\
\hline \multirow[t]{2}{*}{$D=2$} & $\begin{array}{l}L_{K}> \\
L_{K}=\end{array}$ & $\begin{array}{l}L_{0}, L_{100}, L_{250}, L_{500}, L_{750}, L_{1000}, L_{1250} \text {, } \\
L_{1500}\end{array}$ & $\begin{array}{l}L_{K}> \\
L_{K}=\end{array}$ & $L_{0}, L_{100}, L_{500}, L_{1250}, L_{1500}$ & $\begin{array}{l}L_{K}> \\
L_{K}=\end{array}$ & $L_{100}, L_{250}, L_{500}, L_{750}, L_{1250}, L_{1500}, L_{\infty}$ \\
\hline & $L_{K}<$ & $L_{\infty}$ & $L_{K}<$ & $L_{250}, L_{750}, L_{1000}, L_{\infty}$ & $L_{K}<$ & $L_{0}, L_{1000}$ \\
\hline$D=5$ & $\begin{array}{l}L_{K}> \\
L_{K}= \\
L_{K}<\end{array}$ & $\begin{array}{l}L_{0}, L_{100}, L_{250}, L_{500}, L_{750}, L_{1000}, L_{1250}, \\
L_{1500}, L_{\infty}\end{array}$ & $\begin{array}{l}L_{K}> \\
L_{K}= \\
L_{K}<\end{array}$ & $\begin{array}{l}L_{0}, L_{100}, L_{250}, L_{500}, L_{750}, L_{1000}, L_{1250}, \\
L_{1500}, L_{\infty}\end{array}$ & $\begin{array}{l}L_{K}> \\
L_{K}= \\
L_{K}<\end{array}$ & $\begin{array}{l}L_{0}, L_{100}, L_{250}, L_{500}, L_{750}, L_{1000}, L_{1250} \text {, } \\
L_{1500}, L_{\infty}\end{array}$ \\
\hline$D=10$ & $\begin{array}{l}L_{K}> \\
L_{K}= \\
L_{K}<\end{array}$ & $\begin{array}{l}L_{0}, L_{100}, L_{250}, L_{500}, L_{750}, L_{1000}, L_{1250}, \\
L_{1500}, L_{\infty}\end{array}$ & $\begin{array}{l}L_{K}> \\
L_{K}= \\
L_{K}<\end{array}$ & $\begin{array}{l}L_{0}, L_{100}, L_{250}, L_{500}, L_{750}, L_{1000}, L_{1250}, \\
L_{1500}, L_{\infty}\end{array}$ & $\begin{array}{l}L_{K}> \\
L_{K}= \\
L_{K}<\end{array}$ & $\begin{array}{l}L_{0}, L_{100}, L_{250}, L_{500}, L_{750}, L_{1000}, L_{1250}, \\
L_{1500}, L_{\infty}\end{array}$ \\
\hline $\mathrm{D}=\mathbf{3 0}$ & $\begin{array}{l}L_{K}> \\
L_{K}= \\
L_{K}<\end{array}$ & $\begin{array}{l}L_{0}, L_{100}, L_{250}, L_{500}, L_{750}, L_{1000}, L_{1250} \text {, } \\
L_{1500}, L_{\infty}\end{array}$ & $\begin{array}{l}L_{K}> \\
L_{K}= \\
L_{K}<\end{array}$ & $\begin{array}{l}L_{0}, L_{100}, L_{250}, L_{500}, L_{750}, L_{1000}, L_{1250} \\
L_{1500}, L_{\infty}\end{array}$ & $\begin{array}{l}L_{K}> \\
L_{K}= \\
L_{K}<\end{array}$ & $\begin{array}{l}L_{0}, L_{100}, L_{250}, L_{500}, L_{750}, L_{1000}, L_{1250}, \\
L_{1500}, L_{\infty}\end{array}$ \\
\hline $\mathrm{D}=\mathbf{5 0}$ & $\begin{array}{l}L_{K}> \\
L_{K}= \\
L_{K}<\end{array}$ & $\begin{array}{l}L_{0}, L_{100}, L_{250}, L_{500}, L_{750}, L_{1000}, L_{1250}, \\
L_{1500}, L_{\infty}\end{array}$ & $\begin{array}{l}L_{K}> \\
L_{K}= \\
L_{K}<\end{array}$ & $\begin{array}{l}L_{0}, L_{100}, L_{250}, L_{500}, L_{750}, L_{1000}, L_{1250}, \\
L_{1500}, L_{\infty}\end{array}$ & $\begin{array}{l}L_{K}> \\
L_{K}= \\
L_{K}<\end{array}$ & $\begin{array}{l}L_{0}, L_{100}, L_{250}, L_{500}, L_{750}, L_{1000}, L_{1250}, \\
L_{1500}, L_{\infty}\end{array}$ \\
\hline
\end{tabular}


Table 48

Horizontal approach, $10^{-12}$

\begin{tabular}{lcccccccccc}
\hline & $L_{K}$ & $L_{0}$ & $L_{100}$ & $L_{250}$ & $L_{500}$ & $L_{750}$ & $L_{1000}$ & $L_{1250}$ & $L_{1500}$ & $L_{\infty}$ \\
\hline $\mathrm{SN}=24 \mathrm{D}=2$ & 1514 & 1203 & 1557 & 1517 & 1517 & 1560 & 1558 & 1458 & 1565 & 1551 \\
$\mathrm{SN}=24 \mathrm{D}=5$ & 1312 & 981 & 1715 & 1591 & 1525 & 1585 & 1551 & 1588 & 1565 & 1588 \\
$\mathrm{SN}=24 \mathrm{D}=10$ & 1367 & 995 & 1100 & 1770 & 1636 & 1630 & 1672 & 1619 & 1570 & 1641 \\
$\mathrm{SN}=24 \mathrm{D}=30$ & 1434 & 1097 & 1097 & 1097 & 1678 & 1787 & 1764 & 1719 & 1695 & 1633 \\
$\mathrm{SN}=24 \mathrm{D}=50$ & 1507 & 1152 & 1152 & 1152 & 1207 & 1704 & 1821 & 1840 & 1785 & 1681 \\
$\mathrm{SN}=50 \mathrm{D}=2$ & 1613 & 1193 & 1555 & 1494 & 1507 & 1480 & 1477 & 1533 & 1636 & 1511 \\
$\mathrm{SN}=50 \mathrm{D}=5$ & 1663 & 981 & 1673 & 1532 & 1497 & 1529 & 1544 & 1528 & 1515 & 1539 \\
$\mathrm{SN}=50 \mathrm{D}=10$ & 1691 & 996 & 1126 & 1691 & 1626 & 1598 & 1619 & 1674 & 1610 & 1558 \\
$\mathrm{SN}=50 \mathrm{D}=30$ & 1748 & 1111 & 1111 & 1111 & 1708 & 1748 & 1726 & 1695 & 1666 & 1626 \\
$\mathrm{SN}=50 \mathrm{D}=50$ & 1782 & 1165 & 1165 & 1165 & 1236 & 1706 & 1782 & 1782 & 1797 & 1701 \\
$\mathrm{SN}=100 \mathrm{D}=2$ & 1603 & 1191 & 1603 & 1475 & 1486 & 1570 & 1544 & 1540 & 1566 & 1525 \\
$\mathrm{SN}=100 \mathrm{D}=5$ & 1588 & 981 & 1675 & 1588 & 1574 & 1542 & 1513 & 1508 & 1565 & 1555 \\
$\mathrm{SN}=100 \mathrm{D}=10$ & 1677 & 1000 & 1100 & 1703 & 1677 & 1609 & 1551 & 1616 & 1599 & 1648 \\
$\mathrm{SN}=100 \mathrm{D}=30$ & 1679 & 1111 & 1111 & 1111 & 1678 & 1743 & 1714 & 1723 & 1679 & 1631 \\
$\mathrm{SN}=100 \mathrm{D}=50$ & 1719 & 1144 & 1144 & 1144 & 1195 & 1700 & 1761 & 1740 & 1758 & 1695 \\
\hline \multicolumn{7}{c}{ (a) Horizontal } & & & &
\end{tabular}

(a) Horizontal approach, $f_{1}, 10^{-12}$

$\begin{array}{llllllllllll}L_{K} & L_{0} & L_{100} & L_{250} & L_{500} & L_{750} & L_{1000} & L_{1250} & L_{1500} & L_{\infty}\end{array}$ $\begin{array}{llllllllllll} & & L_{K} & L_{0} & L_{100} & L_{2} & & & & & & \end{array}$

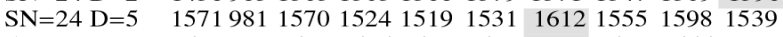

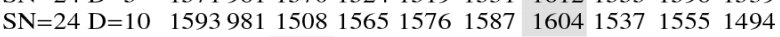

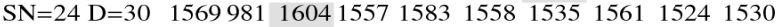

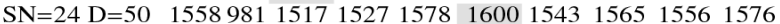

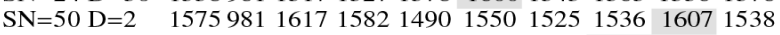

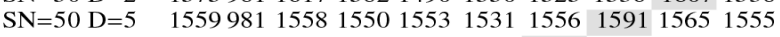

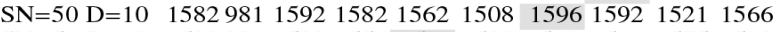

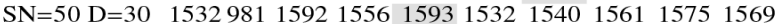

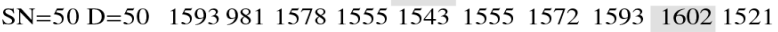

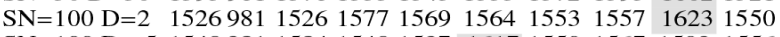

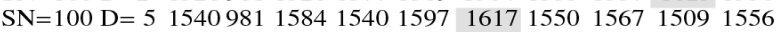
$\mathrm{SN}=100 \mathrm{D}=101571981160015001571 \quad 15331538 \quad 1573 \quad 16181586$

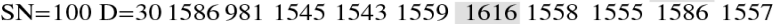
\begin{tabular}{l}
$\mathrm{SN}=100 \mathrm{D}=501576981$ \\
\hline
\end{tabular} (b) Horizontal approach, $f_{2}, 10^{-12}$

\begin{tabular}{lcccccccccc}
\hline & $L_{K}$ & $L_{0}$ & $L_{100}$ & $L_{250}$ & $L_{500}$ & $L_{750}$ & $L_{1000}$ & $L_{1250}$ & $L_{1500}$ & $L_{\infty}$ \\
\hline $\mathrm{SN}=24 \mathrm{D}=2$ & 1541 & 990 & 1562 & 1583 & 1592 & 1560 & 1547 & 1599 & 1529 & 1498 \\
$\mathrm{SN}=24 \mathrm{D}=5$ & 1576 & 981 & 1585 & 1543 & 1523 & 1585 & 1565 & 1566 & 1566 & 1510 \\
$\mathrm{SN}=24 \mathrm{D}=10$ & 1569 & 981 & 1530 & 1588 & 1610 & 1555 & 1557 & 1521 & 1498 & 1591 \\
$\mathrm{SN}=24 \mathrm{D}=30$ & 1528 & 981 & 1582 & 1527 & 1527 & 1569 & 1542 & 1561 & 1583 & 1602 \\
$\mathrm{SN}=24 \mathrm{D}=50$ & 1543 & 981 & 1580 & 1566 & 1568 & 1541 & 1554 & 1595 & 1582 & 1491 \\
$\mathrm{SN}=50 \mathrm{D}=2$ & 1596 & 991 & 1565 & 1591 & 1531 & 1514 & 1532 & 1546 & 1498 & 1636 \\
$\mathrm{SN}=50 \mathrm{D}=5$ & 1603981 & 1578 & 1548 & 1576 & 1515 & 1521 & 1536 & 1566 & 1576 \\
$\mathrm{SN}=50 \mathrm{D}=10$ & 1558 & 981 & 1591 & 1558 & 1529 & 1547 & 1573 & 1548 & 1565 & 1609 \\
$\mathrm{SN}=50 \mathrm{D}=30$ & 1544 & 981 & 1601 & 1539 & 1601 & 1544 & 1570 & 1540 & 1586 & 1538 \\
$\mathrm{SN}=50 \mathrm{D}=50$ & 1548 & 981 & 1556 & 1580 & 1554 & 1529 & 1571 & 1548 & 1587 & 1595 \\
$\mathrm{SN}=100 \mathrm{D}=2$ & 1568 & 985 & 1568 & 1563 & 1556 & 1492 & 1566 & 1573 & 1608 & 1590 \\
$\mathrm{SN}=100 \mathrm{D}=5$ & 1556 & 981 & 1542 & 1556 & 1602 & 1573 & 1582 & 1557 & 1575 & 1533 \\
$\mathrm{SN}=100 \mathrm{D}=10$ & 1552 & 981 & 1539 & 1551 & 1552 & 1601 & 1575 & 1531 & 1637 & 1534 \\
$\mathrm{SN}=100 \mathrm{D}=30$ & 1560 & 981 & 1613 & 1549 & 1556 & 1561 & 1591 & 1504 & 1560 & 1586 \\
$\mathrm{SN}=100 \mathrm{D}=50$ & 1582 & 981 & 1566 & 1595 & 1599 & 1575 & 1528 & 1515 & 1552 & 1508 \\
\hline
\end{tabular}
(c) Horizontal approach, $f_{3}, 10^{-12}$ \begin{tabular}{lcccc|cccccc}
\hline & $L_{K}$ & $L_{0}$ & $L_{100}$ & $L_{250}$ & $L_{500}$ & $L_{750}$ & $L_{1000}$ & $L_{1250}$ & $L_{1500}$ & $L_{\infty}$ \\
\hline $\mathrm{SN}=24 \mathrm{D}=2$ & 1370 & 981 & 1632 & 1584 & 1561 & 1565 & 1599 & 1570 & 1538 & 1600 \\
$\mathrm{SN}=24 \mathrm{D}=5$ & 1583 & 981 & 1517 & 1551 & 1542 & 1543 & 1544 & 1544 & 1625 & 1570 \\
$\mathrm{SN}=24 \mathrm{D}=10$ & 1562 & 981 & 1535 & 1576 & 1521 & 1562 & 1581 & 1602 & 1519 & 1561 \\
$\mathrm{SN}=24 \mathrm{D}=30$ & 1617981 & 1596 & 1544 & 1516 & 1580 & 1561 & 1497 & 1596 & 1513 \\
$\mathrm{SN}=24 \mathrm{D}=50$ & 1543 & 981 & 1521 & 1566 & 1552 & 1595 & 1550 & 1629 & 1493 & 1572 \\
$\mathrm{SN}=50 \mathrm{D}=2$ & 1553 & 981 & 1572 & 1561 & 1558 & 1543 & 1587 & 1566 & 1524 & 1557 \\
$\mathrm{SN}=50 \mathrm{D}=5$ & 1560 & 981 & 1538 & 1588 & 1562 & 1563 & 1531 & 1588 & 1541 & 1548 \\
$\mathrm{SN}=50 \mathrm{D}=10$ & 1530 & 981 & 1551 & 1530 & 1558 & 1636 & 1577 & 1545 & 1580 & 1542 \\
$\mathrm{SN}=50 \mathrm{D}=30$ & 1587 & 981 & 1528 & 1565 & 1578 & 1587 & 1593 & 1574 & 1502 & 1592 \\
$\mathrm{SN}=50 \mathrm{D}=50$ & 1582 & 981 & 1523 & 1568 & 1540 & 1613 & 1565 & 1582 & 1577 & 1552 \\
$\mathrm{SN}=100 \mathrm{D}=2$ & 1587 & 981 & 1587 & 1527 & 1552 & 1579 & 1538 & 1603 & 1571 & 1562 \\
$\mathrm{SN}=100 \mathrm{D}=5$ & 1512 & 981 & 1590 & 1512 & 1539 & 1555 & 1584 & 1586 & 1603 & 1551 \\
$\mathrm{SN}=100 \mathrm{D}=10$ & 1571981 & 9514 & 1574 & 1571 & 1544 & 1612 & 1522 & 1583 & 1599 \\
$\mathrm{SN}=100 \mathrm{D}=30$ & 1618981 & 1608 & 1578 & 1539 & 1548 & 1538 & 1571 & 1618 & 1519 \\
$\mathrm{SN}=100 \mathrm{D}=50$ & 1490 & 981 & 1501 & 1533 & 1591 & 1555 & 1553 & 1577 & 1605 & 1613 \\
\hline
\end{tabular}

(d) Horizontal approach, $f_{4}, 10^{-12}$

\begin{tabular}{lcccccccccc}
\hline & $L_{K}$ & $L_{0}$ & $L_{100}$ & $L_{250}$ & $L_{500}$ & $L_{750}$ & $L_{1000}$ & $L_{1250}$ & $L_{1500}$ & $L_{\infty}$ \\
\hline $\mathrm{SN}=24 \mathrm{D}=2$ & 1500 & 1500 & 1500 & 1500 & 1500 & 1500 & 1500 & 1500 & 1500 & 1500 \\
$\mathrm{SN}=24 \mathrm{D}=5$ & 1500 & 1500 & 1500 & 1500 & 1500 & 1500 & 1500 & 1500 & 1500 & 1500 \\
$\mathrm{SN}=24 \mathrm{D}=10$ & 1500 & 1500 & 1500 & 1500 & 1500 & 1500 & 1500 & 1500 & 1500 & 1500 \\
$\mathrm{SN}=24 \mathrm{D}=30$ & 1500 & 1500 & 1500 & 1500 & 1500 & 1500 & 1500 & 1500 & 1500 & 1500 \\
$\mathrm{SN}=24 \mathrm{D}=50$ & 1500 & 1500 & 1500 & 1500 & 1500 & 1500 & 1500 & 1500 & 1500 & 1500 \\
$\mathrm{SN}=50 \mathrm{D}=2$ & 1500 & 1500 & 1500 & 1500 & 1500 & 1500 & 1500 & 1500 & 1500 & 1500 \\
$\mathrm{SN}=50 \mathrm{D}=5$ & 1500 & 1500 & 1500 & 1500 & 1500 & 1500 & 1500 & 1500 & 1500 & 1500 \\
$\mathrm{SN}=50 \mathrm{D}=10$ & 1500 & 1500 & 1500 & 1500 & 1500 & 1500 & 1500 & 1500 & 1500 & 1500 \\
$\mathrm{SN}=50 \mathrm{D}=30$ & 1500 & 1500 & 1500 & 1500 & 1500 & 1500 & 1500 & 1500 & 1500 & 1500 \\
$\mathrm{SN}=50 \mathrm{D}=50$ & 1500 & 1500 & 1500 & 1500 & 1500 & 1500 & 1500 & 1500 & 1500 & 1500 \\
$\mathrm{SN}=100 \mathrm{D}=2$ & 1500 & 1500 & 1500 & 1500 & 1500 & 1500 & 1500 & 1500 & 1500 & 1500 \\
$\mathrm{SN}=100 \mathrm{D}=5$ & 1500 & 1500 & 1500 & 1500 & 1500 & 1500 & 1500 & 1500 & 1500 & 1500 \\
$\mathrm{SN}=100 \mathrm{D}=10$ & 1500 & 1500 & 1500 & 1500 & 1500 & 1500 & 1500 & 1500 & 1500 & 1500 \\
$\mathrm{SN}=100 \mathrm{D}=30$ & 1500 & 1500 & 1500 & 1500 & 1500 & 1500 & 1500 & 1500 & 1500 & 1500 \\
$\mathrm{SN}=100 \mathrm{D}=50$ & 1500 & 1500 & 1500 & 1500 & 1500 & 1500 & 1500 & 1500 & 1500 & 1500 \\
\hline
\end{tabular}

(e) Horizontal approach, $f_{5}, 10^{-12}$

\subsubsection{Discussion}

The results between CRS4EAs and NHST were comparable. When smaller numbers of fitness evaluations were available, $L_{k}$ was an appropriate choice only for small dimensions; and when sufficiently large enough numbers of fitness evaluations were available, $L_{k}$ was a significantly better choice than the presented fixed 'limit' values. When it was of interest to find a (sub-) optimal solution and large numbers of fitness evaluations were available, better alternatives than $L_{k}$ were available several times. The main difference between NHST and CRS4EAs comparison was that CRS4EAs was more conservative and detected less significant differences than NHST, however, the conservativity/liberality can be easily controlled through rating deviation $R D$ [48]. Otherwise, the methods showed the same trends when and for which population size and dimension $L_{k}$ was an unsuitable choice and when it was a suitable choice. Hence, the main conclusion as presented in Section 2.2.5 is that using NHST was the same as using CRS4EAs. The main differences between both methods showed in the abilities to detect differences amongst fixed 'limit' values. Whilst for NHST only the differences between $L_{k}$ and other fixed 'limit' values were calculated and detected, CRS4EAs allowed direct comparisons between fixed 'limit' values. In order to find the differences between the fixed 'limit' values in NHST, additional tests would be needed, which would be both time consuming and require special care to avoid Type-I-Error.

\section{ABC Parameter Tuning}

In the previous section, it was shown that $A B C$ does not always perform best when under the setting 'limit' 
$=n_{e}{ }^{*} D$. Hence, the 'limit' control parameter should be tuned or controlled. Therefore, this section displays the results of $A B C$ tuning in contrast to the suggested 'limit' setting and to the statistical analysis in Section 2.

Tuning is a process of finding those parameter values for which the meta-heuristic algorithm performs the best for selected sets of problems $F$. A combination of different parameter values is called configuration. One of the more common and easy-to-apply tuning methods is F-Race [4], which empirically evaluates a set of parameter values and discards the bad ones as soon as statistically sufficient evidence - supported by the Friedman test [13], [14] - is gathered against them.

Table 49

$f_{1}$, horizontal approach, $10^{-12}$, CRS4EAs

\begin{tabular}{|c|c|c|c|c|c|c|}
\hline & & $\mathrm{SN}=24$ & & $\mathrm{SN}=50$ & & $\mathrm{SN}=100$ \\
\hline \multirow[b]{2}{*}{$D=2$} & $L_{K}>$ & $L_{0} *, L_{1250}$ & $L_{K}>$ & $\begin{array}{l}L_{0} *, L_{100}, L_{250}, L_{500}, L_{750}, L_{1000}, L_{1250}, \\
L_{\infty},\end{array}$ & $L_{K}>$ & $\begin{array}{l}L_{0 *}, L_{250}, L_{500}, L_{750}, L_{1000}, L_{1250}, \\
L_{1500}, L_{\infty}\end{array}$ \\
\hline & $\begin{array}{l}L_{K}= \\
L_{K}<\end{array}$ & $L_{100}, L_{250}, L_{500}, L_{750}, L_{1000}, L_{1500}, L_{\infty}$ & $\begin{array}{l}L_{K}= \\
L_{K}<\end{array}$ & $L_{1500}$ & $\begin{array}{l}L_{K}= \\
L_{K}<\end{array}$ & \\
\hline \multirow[b]{2}{*}{$D=5$} & $L_{K}>$ & $L_{0 *}$ & $L_{K}>$ & $\begin{array}{l}L_{0} *, \quad L_{250}, L_{500}, L_{750}, L_{1000}, L_{1250}, \\
L_{1500}, L_{\infty}\end{array}$ & $L_{K}>$ & $L_{0} *, L_{500}, L_{750}, L_{1000}, L_{1250}, L_{1500}, L_{\infty}$ \\
\hline & $\begin{array}{l}L_{K}= \\
L_{K}<\end{array}$ & $\begin{array}{l}L_{100 *}, \quad L_{250 *}, \quad L_{500 *}, \quad L_{750 *}, L_{1000 *}, \\
L_{1250 *,} L_{1500^{*},} L_{\infty} *\end{array}$ & $\begin{array}{l}L_{K}= \\
L_{K}<\end{array}$ & 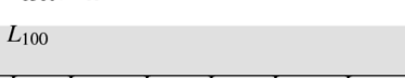 & $\begin{array}{l}L_{K}= \\
L_{K}<\end{array}$ & $\begin{array}{l}L_{250} \\
L_{100}\end{array}$ \\
\hline \multirow[b]{2}{*}{$\mathrm{D}=\mathbf{1 0}$} & $L_{K}>$ & $L_{0} *, L_{100 *}$ & $L_{K}>$ & $\begin{array}{l}L_{0} *, L_{100} *, L_{500}, L_{750}, L_{1000}, L_{1250}, \\
L_{1500}, L_{\infty}\end{array}$ & $L_{K}>$ & $L_{0} *, L_{100} *, L_{750}, L_{1000}, L_{1250}, L_{1500}, L_{\infty}$ \\
\hline & $\begin{array}{l}L_{K}= \\
L_{K}<\end{array}$ & $\begin{array}{l}L_{250 *}, L_{500 *}, L_{750} *, L_{1000 *,} L_{1250 *} \\
L_{1500^{*}, L_{\infty} *}\end{array}$ & $\begin{array}{l}L_{K}= \\
L_{K}<\end{array}$ & $L_{250}$ & $\begin{array}{l}L_{K}= \\
L_{K}<\end{array}$ & $\begin{array}{l}L_{500} \\
L_{250}\end{array}$ \\
\hline $\mathbf{D}=\mathbf{3 0}$ & $\begin{array}{l}L_{K}> \\
L_{K}= \\
L_{K}<\end{array}$ & $L_{500} *, L_{750} *, L_{1000} *, L_{1250} *, L_{1500} *, L_{\infty}$ & $\begin{array}{l}L_{K}> \\
L_{K}= \\
L_{K}<\end{array}$ & $\begin{array}{l}L_{0} *, L_{100} *, L_{250} *, L_{500}, L_{1000}, L_{1250}, \\
L_{1500}, L_{\infty} \\
L_{750}\end{array}$ & $\begin{array}{l}L_{K}> \\
L_{K}= \\
L_{K}<\end{array}$ & $\begin{array}{l}L_{0} *, L_{100} *, L_{250} *, L_{500}, L_{\infty} \\
L_{1500} \\
L_{750}, L_{1000}, L_{1250}\end{array}$ \\
\hline $\mathrm{D}=\mathbf{5 0}$ & $\begin{array}{l}L_{K}> \\
L_{K}= \\
L_{K}<\end{array}$ & $\begin{array}{l}L_{0} *, L_{100} *, L_{250} *, L_{500} * \\
L_{750}, L_{1000} * L_{1250} *, L_{1500 *, L_{\infty}}\end{array}$ & $\begin{array}{l}L_{K}> \\
L_{K}= \\
L_{K}<\end{array}$ & $\begin{array}{l}L_{0} *, L_{100} *, L_{250} *, L_{500} *, L_{750}, L_{\infty} \\
L_{1000}, L_{1250} \\
L_{1500}\end{array}$ & $\begin{array}{l}L_{K}> \\
L_{K}= \\
L_{K}<\end{array}$ & $\begin{array}{l}L_{0} *, L_{100} *, L_{250} *, L_{500} *, L_{750}, L_{\infty} \\
L_{1000}, L_{1250}, L_{1500}\end{array}$ \\
\hline
\end{tabular}

Table 50

$f_{2}$, horizontal approach, $10^{-12}$, CRS4EAs

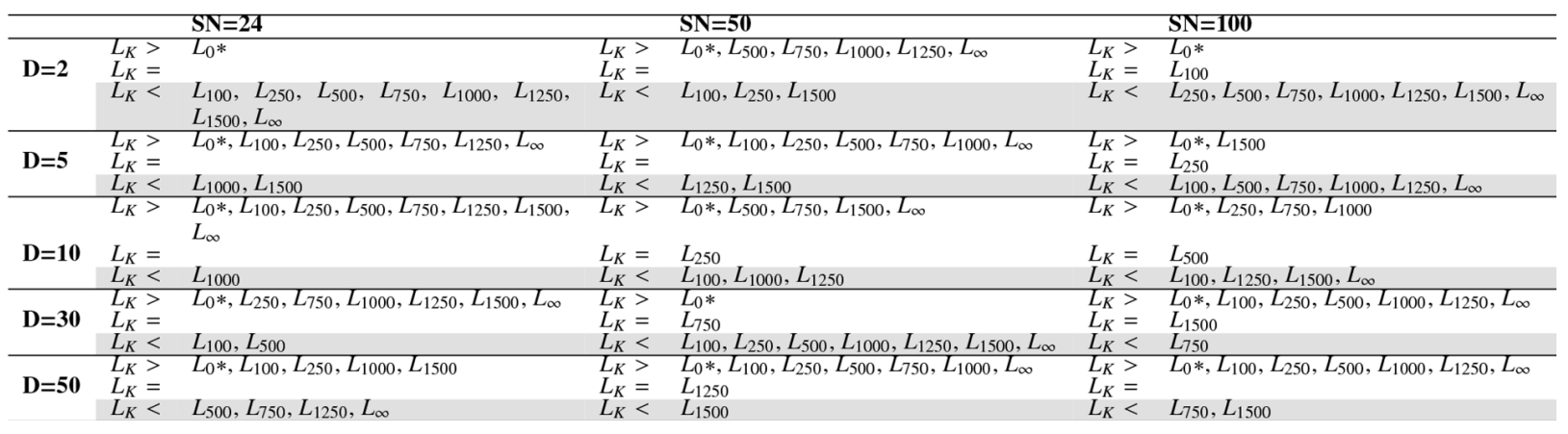

Table 51

$f_{3}$, horizontal approach, $10^{-12}$, CRS4EAs

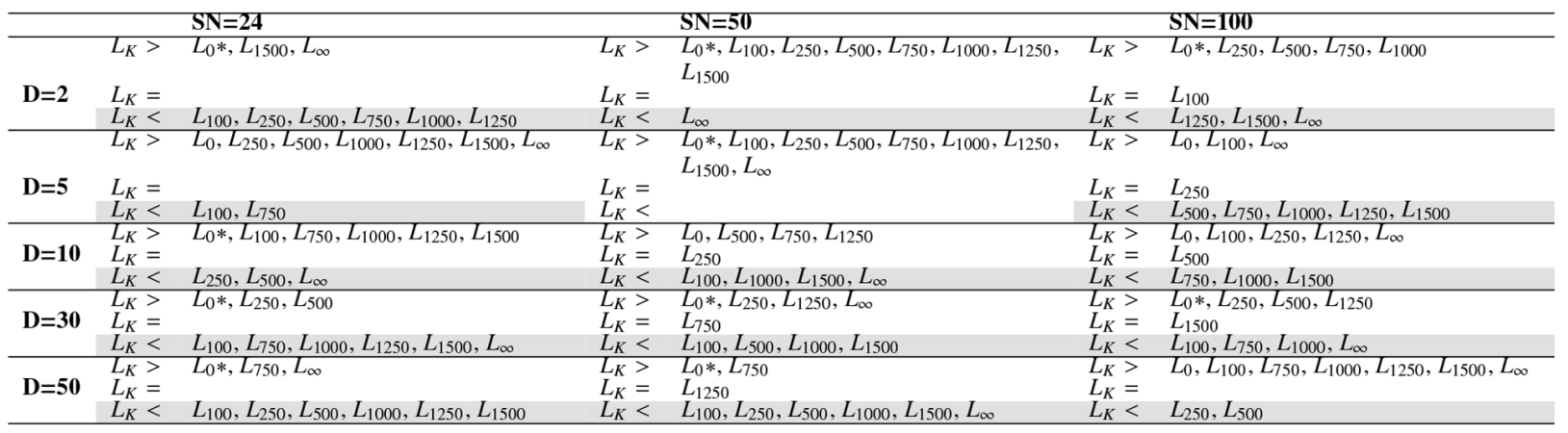




\section{Table 52}

$f_{4}$, horizontal approach, $10^{-12}$, CRS4EAs

\begin{tabular}{|c|c|c|c|c|c|c|}
\hline & & $\mathrm{SN}=24$ & & $\mathrm{SN}=50$ & & $\mathrm{SN}=100$ \\
\hline \multirow[t]{2}{*}{$\mathrm{D}=2$} & $\begin{array}{l}L_{K}> \\
L_{K}=\end{array}$ & $L_{0} *$ & $\begin{array}{l}L_{K}> \\
L_{K}=\end{array}$ & $L_{0} *, L_{750}, L_{1500}$ & $\begin{array}{l}L_{K}> \\
L_{K}=\end{array}$ & $\begin{array}{l}L_{0} *, L_{250}, L_{500}, L_{750}, L_{1000}, L_{1500}, L_{\infty} \\
L_{100}\end{array}$ \\
\hline & $L_{K}<$ & $\begin{array}{l}L_{100} *, L_{250} *, L_{500}, L_{750}, L_{1000} *, L_{1250}, \\
L_{1500}, L_{\infty} *\end{array}$ & $L_{K}<$ & $L_{100}, L_{250}, L_{500}, L_{1000}, L_{1250}, L_{\infty}$ & $L_{K}^{n}<$ & $L_{1250}$ \\
\hline \multirow{3}{*}{$D=5$} & $L_{K}>$ & $\begin{array}{l}L_{0} *, L_{100}, L_{250}, L_{500}, L_{750}, L_{1000}, L_{1250}, \\
L_{\infty}\end{array}$ & $L_{K}>$ & $L_{0} *, L_{100}, L_{1000}, L_{1500}, L_{\infty}$ & $L_{K}>$ & $L_{0}$ \\
\hline & $L_{K}=$ & & $L_{K}=$ & & $L_{K}=$ & $L_{250}$ \\
\hline & $L_{K}<$ & $L_{1500}$ & $L_{K}<$ & $L_{250}, L_{500}, L_{750}, L_{1250}$ & $L_{K}<$ & $L_{100}, L_{500}, L_{750}, L_{1000}, L_{1250}, L_{1500}, L_{\infty}$ \\
\hline \multirow[t]{2}{*}{$\mathrm{D}=10$} & $\begin{array}{l}L_{K}> \\
L_{K}=\end{array}$ & $L_{0} *, L_{100}, L_{500}, L_{750}, L_{1500}, L_{\infty}$ & $\begin{array}{l}L_{K}> \\
L_{K}=\end{array}$ & $\begin{array}{l}L_{0} * \\
L_{250}\end{array}$ & $\begin{array}{l}L_{K}> \\
L_{K}=\end{array}$ & $\begin{array}{l}L_{0} *, L_{100}, L_{750}, L_{1250} \\
L_{500}\end{array}$ \\
\hline & $L_{K}<$ & $L_{250}, L_{1000}, L_{1250}$ & $L_{K}<$ & $L_{100}, L_{500}, L_{750}, L_{1000}, L_{1250}, L_{1500}, L_{\infty}$ & $L_{K}<$ & $L_{250}, L_{1000}, L_{1500}, L_{\infty}$ \\
\hline \multirow[b]{2}{*}{$D=30$} & $L_{K}>$ & $\begin{array}{l}L_{0} *, L_{100}, L_{250}, L_{500}, L_{750}, L_{1000}, L_{1250}, \\
L_{1500}, L_{\infty}\end{array}$ & $L_{K}>$ & $L_{0} *, L_{100}, L_{250}, L_{500}, L_{1250}, L_{1500}$ & $L_{K}>$ & $\begin{array}{l}L_{0} *, L_{100}, L_{250}, L_{500}, L_{750}, L_{1000}, L_{1250} \\
L_{\infty}\end{array}$ \\
\hline & $\begin{array}{l}L_{K}= \\
L_{K}<\end{array}$ & & $\begin{array}{l}L_{K}= \\
L_{K}<\end{array}$ & $\begin{array}{l}L_{750} \\
L_{1000}, L_{\infty}\end{array}$ & $\begin{array}{l}L_{K}= \\
L_{K}<\end{array}$ & $L_{1500}^{\infty}$ \\
\hline \multirow[t]{2}{*}{$\mathrm{D}=\mathbf{5 0}$} & $\begin{array}{l}L_{K}> \\
L_{K}=\end{array}$ & $L_{0} *, L_{100}, L_{1500}$ & $\begin{array}{l}L_{K}> \\
L_{K}=\end{array}$ & $\begin{array}{l}L_{0} *, L_{100}, L_{250}, L_{500}, L_{1000}, L_{1500}, L_{\infty} \\
L_{1250}\end{array}$ & $\begin{array}{l}L_{K}> \\
L_{K}=\end{array}$ & $L_{0} *$ \\
\hline & $L_{K}<$ & $L_{250}, L_{500}, L_{750}, L_{1000}, L_{1250}, L_{\infty}$ & $L_{K}<$ & $L_{750}$ & $L_{K}<$ & $\begin{array}{l}L_{100}, L_{250}, L_{500}, L_{750}, L_{1000}, L_{1250} \\
L_{1500}, L_{\infty}\end{array}$ \\
\hline
\end{tabular}

\section{Table 53}

$f_{5}$, horizontal approach, $10^{-12}$, CRS4EAs

\begin{tabular}{|c|c|c|c|c|c|c|}
\hline & & $\mathrm{SN}=24$ & & $\mathrm{SN}=\mathbf{5 0}$ & & $\mathrm{SN}=100$ \\
\hline $\mathrm{D}=2$ & $\begin{array}{l}L_{K}> \\
L_{K}= \\
L_{K}<\end{array}$ & $\begin{array}{l}L_{0}, L_{100}, L_{250}, L_{500}, L_{750}, L_{1000}, L_{1250} \\
L_{1500}, L_{\infty}\end{array}$ & $\begin{array}{l}L_{K}> \\
L_{K}= \\
L_{K}<\end{array}$ & $\begin{array}{l}L_{0}, L_{100}, L_{250}, L_{500}, L_{750}, L_{1000}, L_{1250} \\
L_{1500}, L_{\infty}\end{array}$ & $\begin{array}{l}L_{K}> \\
L_{K}= \\
L_{K}<\end{array}$ & $\begin{array}{l}L_{0}, L_{100}, L_{250}, L_{500}, L_{750}, L_{1000}, L_{1250}, \\
L_{1500}, L_{\infty}\end{array}$ \\
\hline $\mathrm{D}=5$ & $\begin{array}{l}L_{K}> \\
L_{K}= \\
L_{K}<\end{array}$ & $\begin{array}{l}L_{0}, L_{100}, L_{250}, L_{500}, L_{750}, L_{1000}, L_{1250} \\
L_{1500}, L_{\infty}\end{array}$ & $\begin{array}{l}L_{K}> \\
L_{K}= \\
L_{K}<\end{array}$ & $\begin{array}{l}L_{0}, L_{100}, L_{250}, L_{500}, L_{750}, L_{1000}, L_{1250} \\
L_{1500}, L_{\infty}\end{array}$ & $\begin{array}{l}L_{K}> \\
L_{K}= \\
L_{K}<\end{array}$ & $\begin{array}{l}L_{0}, L_{100}, L_{250}, L_{500}, L_{750}, L_{1000}, L_{1250}, \\
L_{1500}, L_{\infty}\end{array}$ \\
\hline$D=10$ & $\begin{array}{l}L_{K}> \\
L_{K}= \\
L_{K}<\end{array}$ & $\begin{array}{l}L_{0}, L_{100}, L_{250}, L_{500}, L_{750}, L_{1000}, L_{1250} \\
L_{1500}, L_{\infty}\end{array}$ & $\begin{array}{l}L_{K}> \\
L_{K}= \\
L_{K}<\end{array}$ & $\begin{array}{l}L_{0}, L_{100}, L_{250}, L_{500}, L_{750}, L_{1000}, L_{1250} \\
L_{1500}, L_{\infty}\end{array}$ & $\begin{array}{l}L_{K}> \\
L_{K}= \\
L_{K}<\end{array}$ & $\begin{array}{l}L_{0}, L_{100}, L_{250}, L_{500}, L_{750}, L_{1000}, L_{1250}, \\
L_{1500}, L_{\infty}\end{array}$ \\
\hline$D=30$ & $\begin{array}{l}L_{K}> \\
L_{K}= \\
L_{K}<\end{array}$ & $\begin{array}{l}L_{0}, L_{100}, L_{250}, L_{500}, L_{750}, L_{1000}, L_{1250} \\
L_{1500}, L_{\infty}\end{array}$ & $\begin{array}{l}L_{K}> \\
L_{K}= \\
L_{K}<\end{array}$ & $\begin{array}{l}L_{0}, L_{100}, L_{250}, L_{500}, L_{750}, L_{1000}, L_{1250} \\
L_{1500}, L_{\infty}\end{array}$ & $\begin{array}{l}L_{K}> \\
L_{K}= \\
L_{K}<\end{array}$ & $\begin{array}{l}L_{0}, L_{100}, L_{250}, L_{500}, L_{750}, L_{1000}, L_{1250}, \\
L_{1500}, L_{\infty}\end{array}$ \\
\hline$D=50$ & $\begin{array}{l}L_{K}> \\
L_{K}= \\
L_{K}<\end{array}$ & $\begin{array}{l}L_{0}, L_{100}, L_{250}, L_{500}, L_{750}, L_{1000}, L_{1250} \\
L_{1500}, L_{\infty}\end{array}$ & $\begin{array}{l}L_{K}> \\
L_{K}= \\
L_{K}<\end{array}$ & $\begin{array}{l}L_{0}, L_{100}, L_{250}, L_{500}, L_{750}, L_{1000}, L_{1250} \\
L_{1500}, L_{\infty}\end{array}$ & $\begin{array}{l}L_{K}> \\
L_{K}= \\
L_{K}<\end{array}$ & $\begin{array}{l}L_{0}, L_{100}, L_{250}, L_{500}, L_{750}, L_{1000}, L_{1250}, \\
L_{1500}, L_{\infty}\end{array}$ \\
\hline
\end{tabular}

Before the tuning procedure starts, the user has to define the initial set $P$ of all configurations that will be tested, number of initial races $r$, significance level $\alpha$ under which the statistical tests will be applied, and maximum number of executions. In each iteration, all configurations from $P$ will be executed on one random problem from the set $F$ over $n_{f}$ independent runs. After that, if the number of iterations is greater than $r$, a Friedman test will be applied to see if there are significant differences amongst all configurations in $P$. If the Friedman test shows that there are significant differences, a post-hoc test, such as Holm test [19] is applied between the best performing configuration (the one with the smallest Friedman rank) and other configurations. Those configurations that are significantly worse than the best performing configuration under significance level $\alpha$ are removed from set $P$. This procedure is repeated until the maximum number of executions is reached or only one configuration remains in $P$ (Algorithm 2). As already described, one execution is treated as the execution of one configuration on one problem from $F$ over $n_{f}$ independent runs.

To test the suggested formula for parameter 'limit' further, we tuned parameters $S N$ and 'limit' for different dimensions $D$ on problem $f_{1}$ with vertical approach and maximum number of fitness evaluations 100,000. The number of independent runs $n_{f}$ $=25$, the number of initial races $r=5$, significance level $\alpha=0.05$, and maximum number of executions 
equaled 15,000. A goal of this experiment was to find the parameter values $S N$ and 'limit' for which $\mathrm{ABC}$ will perform the best on $f_{1}$ for different dimensions. Hence, some boundaries and precisions of these two parameters needed to be set. The values parameter $S N$ could take were $\{10,20,30, \ldots, 100\}$, and the values parameter 'limit' could take were $\{0,50,100,150, \ldots$, $1450,1500, \infty\}$. These values are different from those used in the experiment in Section 2, as the goal of this experiment is different as well. In this experiment, we wanted to tune the parameters of $A B C$ and in the experiment from Section 2 the goal was to make a pairwise comparison of pre-selected values. In other words, the values of $S N$ and $D$ were fixed in Section 2 and the performances of different 'limit' settings compared to Karaboga's 'limit' setting. In this section, on the other hand, only the allowed values of $S N$ and 'limit' were defined, and the best settings of $S N$ and 'limit' for each fixed value of dimension $D$ were selected with a tuning process. The size of the initial population $P$ equaled 320 (10*32 combinations, 10 for $S N$ and 32 for 'limit'). The conclusions of the tuning process are summarised as follows.

- When the dimensionality of a problem was set to $D=$ 2,62 configurations remained from the initial set $P$. The values of parameter $S N$ were from 20 to 100 , and the values of parameter 'limit' were from 100 to 500 . The best performing configurations (those with the lowest Friedman ranks) were $\{S N=60$, 'limit' $=200\},\{S N=40$, 'limit' $=200\}$, and $\{S N=80$, 'limit' $=200\}$. Following the Karaboga's formula, the ratio between 'limit' and $S N$ when $D=2$ should be 1:1, meaning that 'limit' should have the same value as $S N$. None of the configurations found by the tuning process corresponded to this formula.

- When the dimensionality of a problem was set to $D=5,19$ configurations remained from the initial set $P$. The values of parameter $S N$ were from 30 to 50 , and the values of parameter 'limit' were from 300 to 700 . The best performing configurations were $\{S N=30$, 'limit' $=300\},\{S N=40$, 'limit' = $400\}$, and $\{S N=40$, 'limit' $=550\}$. Following the Karaboga's formula, the ratio between 'limit' and $S N$ when $D=5$ should be 2.5:1, meaning that 'limit' should be 2.5-times greater than $S N$. None of the configurations found by the tuning process corresponded to this formula.

\section{Table 54}

Horizontal approach, $10^{-6}$, large dimension

$L_{K} \quad L_{0} L_{1000} L_{2000} L_{3000} L_{4000} L_{5000} L_{6000} L_{7000} L_{8000} L_{9000} L_{10000} L_{11000} L_{12000} L_{13000} L_{14000} L_{15000} L_{\text {inf }}$ SN=24 D=100 14279831277157715761537153115661629148216091587161715241526151515321504 SN=24D=200 145010311031125415031543161216041603161115651590152916521606163415841597 $S N=24 D=300 \quad 141811651165116513371467154715911645162016131597167616111580163916121552$ SN=50 D=100 14879831236156815271642156915561556152515481539149515421557155715851529 SN=50D=200 163410331033116815181547163416091605155915781582159716191503161016091564 SN=50D=300 162211431143115613051511156716311670160116241574154215321536160716331605 SN=100 D=100 15279841278147215561585152715351574161115611520159215721514156315291501 SN=100 D=200158810291029121715331605159115831604161516271588 166715351573152915601527 SN=100D=300162011461146114613281543156115501579160015971630153616191659153916201579

$$
\text { (a) Horizontal approach, } f_{1}, 10^{-6}
$$

$L_{K} \quad L_{0} L_{1000} L_{2000} L_{3000} L_{4000} L_{5000} L_{6000} L_{7000} L_{8000} L_{9000} L_{10000} L_{11000} L_{12000} L_{13000} L_{44000} L_{15000} L_{i n f}$ SN=24D=100 15439811535157115291504147615461499145114971576160415401474156716001507 SN=24D=200 16219811498142315831526148015131588153315041565155715241608146714691561 SN=24 D=300 15339811518152114541494151215491557151014521632154815821535152315261572 SN=50D=100 15439811490151314901520154214991641149015541540149815581542151515531532 SN=50D=200 15219811518157315071540152115671508158314911578152315191538147915401515 SN=50D=300 15679811511150515501612156415901496148615271496149515571481157914821520 SN=100D=100 15319811597145414691491153114981548159415681527150515541526155614981571 SN=100D=200154298115681544157914861546155814801481 14621542162315101489153114741604 SN=100D=300 15879811548146815451582148315021518146715781551154615421522150715871487

$$
\text { (b) Horizontal approach, } f_{2}, 10^{-6}
$$

$L_{K} L_{0} L_{1000} L_{2000} L_{3000} L_{4000} L_{5000} L_{60100} L_{7000} L_{8000} L_{9000} L_{10000} L_{11000} L_{12000} L_{13000} L_{14000} L_{15000} L_{\text {inf }}$ $\overline{\mathrm{SN}=24 \mathrm{D}=100 \quad 14909811594154314771535151715231543147514901531152615891590150915131575}$ SN=24D=200 14859811503157315841526156215711505158614011556153815621502148715671512 SN=24D=300 15449811570155314961544149615461539151515271565149714981529148815701541 SN=50D=100 14789811580145914741604150515481561149215601451156815841538153415381544 SN=50D=200 14749811565148415401547147414971498157516161541150715231556158915111523 SN=50D=300 15079811531155315031553154015531528152014671513148815841559153115811508 SN=100D=100 15239811484155915221512152315401543151115311502149615351545154815701574 SN=100 D=2001540981 1541156314981530154515101511155515931540146916001479153915251482 SN=100D=300 15359811513148914741570150914821551158215651612153114891608148015351491 (c) Horizontal approach, $f_{3}, 10^{-6}$

$L_{K} \quad L_{0} L_{1000} L_{2000} L_{3000} L_{4000} L_{5000} L_{6000} L_{7000} L_{8000} L_{9000} L_{10000} L_{1100} L_{22000} L_{13000} L_{14000} L_{15000} L_{\text {inf }}$ $\overline{\mathrm{SN}=24 \mathrm{D}=10015389811543146215251533149915851573151215531518150215161545150015971520}$ SN=24D=200 146410831573153414371565149214801547149415401563156215271521155214191645 SN=24D=300 150015001500150015001500150015001500150015001500150015001500150015051500 SN=50D=100 14549811509152415071545150915021620143615341643148115321571160015131540 $S N=50 D=200153110931506151515721487153115961526147115441528154714631503146515071616$ SN=50D=300 150015001500150015001500150015001500150015001500150015001500150015051500 SN=100 D=100 15589811525152013661517155815801513155415971571148415821493152716411433 SN=100 D= 200147510821518157815561445160315921501156015301475152515511389156014921568 SN=100 D=300 150015001500150015001500150015001500150015001500150015001500150015051500 (d) Horizontal approach, $f_{4}, 10^{-6}$

$L_{K} \quad L_{0} \quad L_{1000} L_{2000} L_{3000} L_{4000} L_{5000} L_{60000} L_{7000} L_{80000} L_{9000} L_{10000} L_{11000} L_{12000} L_{13000} L_{14000} L_{15000} L_{\text {inf }}$ SN=24 D=100 150015001500150015001500150015001500150015001500150015001500150015051500 $\mathrm{SN}=24 \mathrm{D}=200 \quad 150015001500150015001500150015001500150015001500150015001500150015051500$ SN=24D=300 150015001500150015001500150015001500150015001500150015001500150015051500 SN=50D=100 150015001500150015001500150015001500150015001500150015001500150015051500 $\mathrm{SN}=50 \mathrm{D}=200 \quad 150015001500150015001500150015001500150015001500150015001500150015051500$ $\mathrm{SN}=50 \mathrm{D}=300 \quad 150015001500150015001500150015001500150015001500150015001500150015051500$ SN=100 D=100 150015001500150015001500150015001500150015001500150015001500150015051500 SN=100 D=200150015001500150015001500150015001500150015001500 1500 15001500150015051500 $\mathrm{SN}=100 \mathrm{D}=300150015001500150015001500150015001500150015001500150015001500150015051500$ (e) Horizontal approach, $f_{5}, 10^{-6}$ 


\section{Table 55}

$f_{1}$, horizontal approach, $10^{-6}$, large dimension, CRS4EAs

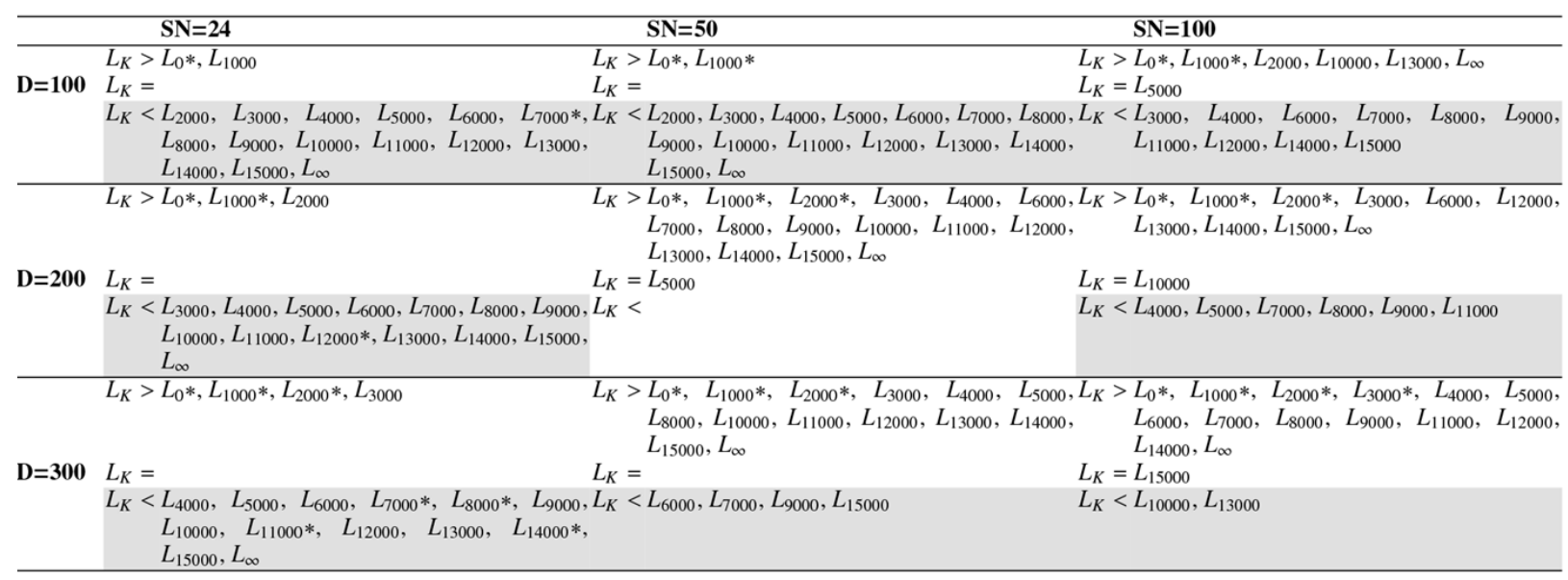

\section{Table 56}

$f_{2}$, horizontal approach, $10^{-6}$, large dimension, CRS4EAs

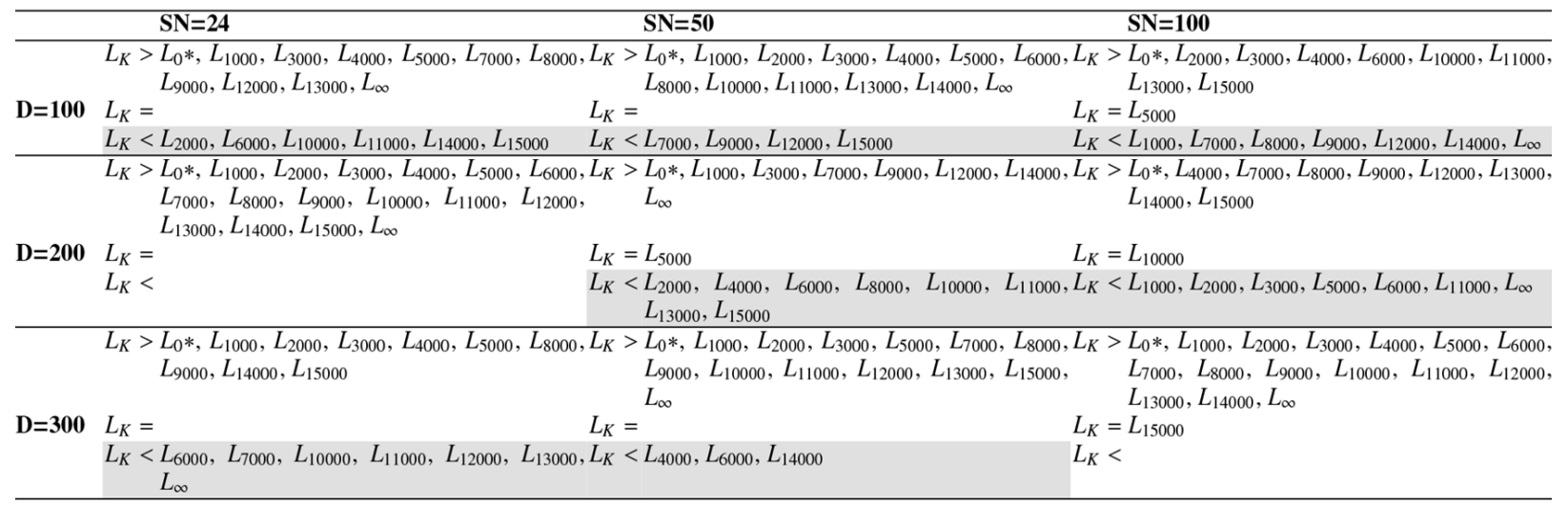

\section{Table 57}

$f_{3}$, horizontal approach, $10^{-6}$, large dimension, CRS4EAs

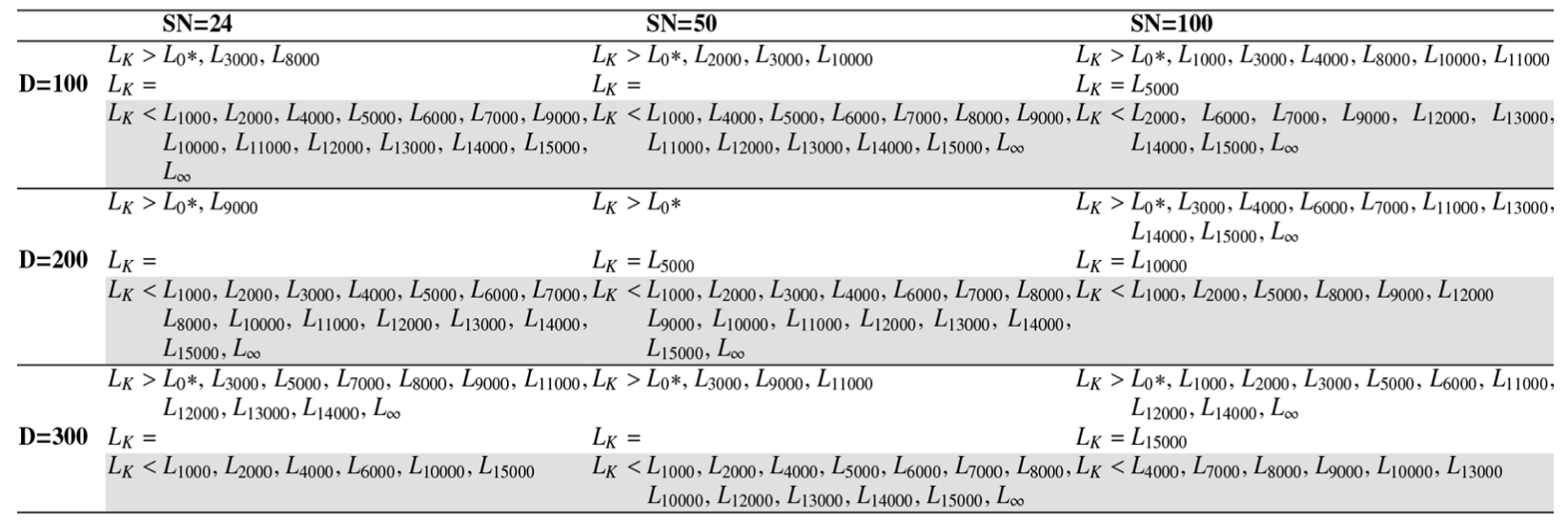


Table 58

$f_{4}$, horizontal approach, $10^{-6}$, large dimension, CRS4EAs

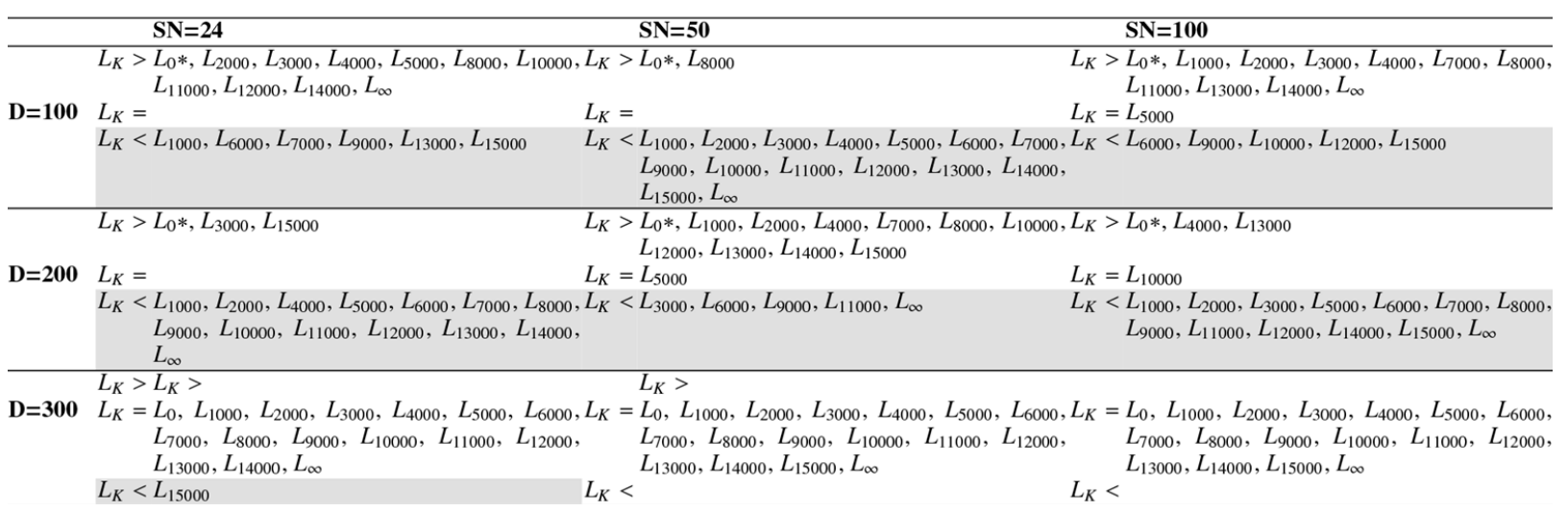

Table 59

$f_{5}$, horizontal approach, $10^{-6}$, large dimension, CRS4EAs

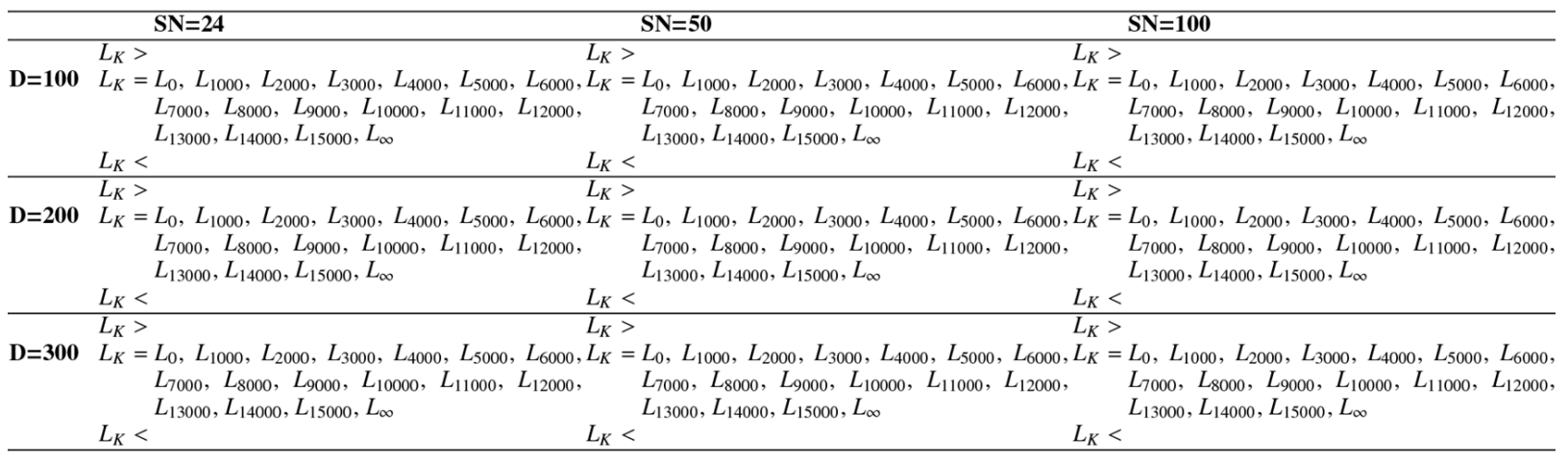

When the dimensionality of a problem was set to $D=10$, four configurations remained from the initial set $P$. The values of parameter $S N$ were 30 , and the values of parameter 'limit' were from 800 to 1000. Those four configurations were $\{S N=30$, 'limit' $=800\},\{S N=30$, 'limit' $=1000\},\{S N=30$, 'limit' $=950\}$, and $\{S N=30$, 'limit' $=850\}$. Following the Karaboga's formula, the ratio between 'limit' and $S N$ when $D=10$ should be 5:1, meaning that 'limit' should be 5 -times greater than $S N$. None of the configurations found by the tuning process corresponded to this formula.

When the dimensionality of a problem was set to $D=30,70$ configurations remained from the initial set $P$. The values of parameter $S N$ were from 10 to 70 , and the values of parameter 'limit' were from 700 to $\infty$. The best performing configurations were $\{S N=20$, 'limit' $=1450\},\{S N=20$, 'limit'
$=1250\}$, and $\{S N=20$, 'limit' $=1500\}$. Following the Karaboga's formula, the ratio between 'limit' and $S N$ when $D=30$ should be $15: 1$, meaning that 'limit' should be 15-times greater than $S N$. None of the configurations found by the tuning process corresponded to this formula.

- When the dimensionality of a problem was set to $D=50,13$ configurations remained from the initial set $P$. The values of parameter $S N$ were from 20 to 40 , and the values of parameter 'limit' were from 1100 to $\infty$. The best performing configurations were $\{S N=20$, 'limit' $=\infty\},\{S N=30$, 'limit' $=\infty\}$, and $\{S N=40$, 'limit' $=\infty\}$. Following the Karaboga's formula, the ratio between 'limit' and $S N$ when $D=50$ should be 25:1, meaning that 'limit' should be 25-times greater than $S N$. None of the configurations found by the tuning process corresponded to this formula. 
One would expect that these results can be compared to those in Tables 5 and 31, however as Tables 5 and 31 display the answers to different questions (as already explained above), the results and conclusions of these experiments cannot be compared directly. There are, however, some similarities between the conclusions of both sections. For example, when $D=10$ it can be noticed that F-Race found the following best configurations: $S N=30$ and 'limit' $=\{800,950,1000\}$. Whilst, from Tables 5 and 31 it can be noticed that configurations with $S N=24$ and 'limit' $=\{750,1000,1250\}$ are significantly better statistically than $L_{k}$ under NHST and CRS4EAs. Or, when $D=30$ it can be noticed that F-Race recommended the following best configurations: $S N=20$ and 'limit' $=\{1250,1450,1500\}$. Whilst, from Tables 5 and 31 it can be noticed that configurations with $S N=24$ and 'limit' $=\{1000,1250,1500\}$ are significantly better statistically than $L_{k}$ under NHST and only better, but not statistically significant, under CRS4EAs as CRS4EAs is more conservative than NHST in this experiment.

Overall, the results of ABC parameter tuning showed that the best performing configurations did not correspond to the Karaboga's formula for $f_{1}$. Similar conclusion can be derived from recent study [49] where for $\mathrm{ABC}$ parameter tuning F-Race, Revac, and CRS-Tuning have been used. From the best performing configurations found by F-Race, Revac, and CRS-Tuning none conform to the Karaboga formula.

\section{Related Work}

To date there have been no deep investigations about setting $A B C$ control parameter 'limit'. The formula 'limit' $=n_{e}{ }^{*} D$ was first proposed in the ABC introductory paper [29] and since then used in many papers (e.g., [6], [21], [24], [27], [28], [31], [54]). The effect of 'limit', as investigated by ABC inventors [29], has been studied on the same benchmark suite $f_{1}, \ldots, f_{5}$ as presented in Section 2 (actually we used the same benchmark suite as in [29]) using the following factors and their values: $S N=\{20,40,100\}, D=\{2,5,50\}$, and 'limit' $=\left\{0.1^{*} n_{e}{ }^{*} D, 0.5^{*} n_{e}{ }^{*} D, n_{e}{ }^{*} D, \infty\right\}$. However, full factorial design has not been used since $D=2$ was used only for $f_{1}, D=5$ for $f_{2}$, and $D=50$ for $f_{3}, \ldots, f_{5}$. Furthermore, the only vertical approaches applied in [26] used 20,000 fitness evaluations for $f_{1}, f_{2}$, and 100,000 fitness evaluations for $f_{3}, \ldots, f_{5}$. In our work, Karaboga's experiment [8] has been extended by performing a full factorial design on this benchmark suite using the following factors and their values: $S N=\{24,50$, $100\}, D=\{2,5,10,30,50\}$, and 'limit' $=\{0,100,250$, $500,750,1000,1250,1500, \infty\}$, whilst using two different horizontal and vertical approaches [18]. The other difference between these two studies is that 30 independent runs were used in [8], whilst 100 in our study in order to enhance its reliability.

The effect of 'limit' on ABC was briefly studied in [1] on functions $f_{1} \ldots f_{5}$, Ackley and Weierstrass with a vertical approach (30,000 fitness evaluations, 30 independent runs) using the following factors and their values: $S N=\{10\}, D=\{10\}$, and 'limit' $=\{10,200,500$, $1000,3000,5000\}$. It was found that 'limit' $=200$ was more appropriate than other values used in this study. Again, our study can be seen as an extension of [1].

A similar study as in [1] on the effect of 'limit' has been recently performed in [30] using a variant of $A B C$ called the quick artificial bee colony (qABC) algorithm. The vertical approach has been applied with 500,000 function evaluations and 30 independent runs on a benchmark suite containing optimisation functions $f_{2}, \ldots, f_{5}$. The following factors and their values have been used: $S N=\{50\}, D=\{30\}$, and 'limit' $=\{10,50,187,375,750,1500\}$. It was found that the 'limit' $=750$ is the more suitable value, which is equal to the value calculated from the formula 'limit' $=n_{e}{ }^{*} D$.

The Enhancing artificial bee colony (EABC) algorithm has been proposed in [15] and tested on 48 benchmark functions. The effect of 'limit' on EABC was investigated with vertical approach $(150,000$ function evaluations, 30 independent runs) on seven functions out of 48 . The following factors and their values were used: $S N=\{100\}, D=\{30\}$, and 'limit' = $\{50,100,200,400, \infty\}$. It was reported that 'limit' = 200 was the more appropriate than other values used in that study.

All the aforementioned works exhibit partial experimentation and non-full factorial design on investigating the effect of 'limit' on ABC. However, such partial investigations were still better, in our opinion, than using a fixed setting from a study using different optimisation problem. The results from our study show that the tuning or controlling of the control parameter 
'limit' is indeed needed. An example of a study where tuning on 'limit' was applied is presented in [35].

It is worth mentioning that in all of the above mentioned experiments, the better settings for 'limit' were chosen by visual inspection of the results and without any statistical testing. Our study was quite different to the aforementioned works due to the applications of NHST and CRS4EAs. In this respect, our work was similar to [42], where full factorial design and ANOVA statistical analysis were used to investigate the sensitivity of reactive tabu search (RTS) to its meta-parameters.

\section{Conclusions}

As the horse racing approach is still omnipresent, researchers have often compared their algorithms, which are well tuned for (a) particular problem(s), with some standard versions of meta-heuristic algorithms using recommended control parameter settings, which might not be appropriate for some problems used in an experiment. This situation should be avoided. In the recently published guidelines for replication and comparison of experiments in EC [9], we promoted fair comparisons amongst algorithms where all the algorithms used in the comparisons, not only the researchers' preferred, should be using the best control parameter settings. Hence, performing extensive parameter tuning or control [11] for all algorithms involved in an experiment is a prerequisite for a fairer comparison.

This paper has shown that amongst ABC control parameters 'limit' is very sensitive, whilst population size $(S N)$ is quite robust (at least for the benchmark suite used in this study). Hence, properly setting control parameter 'limit' should be of particular interest to every ABC user. Furthermore, it was shown in this study that $A B C$ is not always the best performing when 'limit' $=(S N / 2)^{*} D$, although it is a very competitive setting. This formula was the best for the vertical approach using 250,000 fitness evaluations for the benchmark suite used in this study. Better settings for 'limit' exist, occasionally statistically significant, for the vertical approach using 100,000 fitness evaluations, as well as for the both horizontal approaches (reaching (sub-)optimal solution at $10^{-6}$ and $10^{-12}$ ) for benchmark suite used in this study. When 100,000 fitness evaluations were available, $L_{k}$ was the appropriate choice only for small dimensions $(D=2$, rarely for $D=5$ or $D=10$ ) amongst all the five presented problems. When the dimension becomes bigger, more appropriate alternatives could be chosen. Hence, proper setting of 'limit' also depends on the available maximum number of fitness evaluations, indicating that $\mathrm{ABC}$ convergence with $L_{k}$ is not amongst the fastest. Furthermore, as results from the horizontal approach indicate a better $\mathrm{ABC}$ convergence whilst obtaining the same accuracy can be achieved with 'limit' settings other than using the recommended formulae. Moreover, setting 'limit' $=(S N / 2){ }^{*} D$ has no theoretical explanation in [26] and is based only on partial experimentation on limited number of numerical optimization. Hence, it is too risky to expect that the suggested formula in [26] would be good for other problems. Our recommendation is to perform tuning or control on $\mathrm{ABC}$ parameter 'limit'. These findings are valid for $\mathrm{ABC}$ only, and no generalisations regarding other meta-heuristic algorithms can be applied. As extensive parameter tuning using full factorial design [33] is often too expensive, researchers should use various already-available tuning approaches (e.g., F-Race [4], Revac [40], SPO [3], CRS-Tuning [49]) for setting control parameter 'limit' or investigate some parameter control approaches (e.g., driven by diversity [46], entropy [36], exploration and exploitation measures [37]), which will be part of our future work. Last but not least, it is shown that CRS4EAs is comparable to NHST, in particular to the multiple pairwise Wilcoxon's test. Both methods pairwisely compare the results of an optimisation problem over all $n$ runs. However, in one tournament, the CRS4EAs compared the results obtained by all participants (more absolute approach), whilst the Wilcoxon's test compared only results of the participants that are of the main interest (more relative approach). Thus, several Wilcoxon's tests were applied separately for each and every comparison. Additionally, for a set of Wilcoxon's tests made on the same data, a post-hoc analysis is needed to avoid inflating Type-I-Error. Nevertheless, the results of CRS4EAs can be compared amongst all participants, whilst in NHST even more additional tests would be needed in this respect. Deeper comparison among CRS4EAs and NHST is presented in our recent work [50]. 


\section{References}

1. Akay, B., Karaboga, D. A Modified Artificial Bee Colony Algorithm for Real-Parameter Optimization. Information Sciences, 2012, 192, 120-142. https://doi. org/10.1016/j.ins.2010.07.015

2. Amarjeet, J., Chhabra, K. Harmony Search Based Remodularization for Object-Oriented Software Systems. Computer Languages, Systems \& Structures, 2017, 47, 153-169. https://doi.org/10.1016/j.cl.2016.09.003

3. Bartz-Beielstein, T., Lasarczyk, C., Preuss, M. Sequential Parameter Optimization. The 2005 IEEE Congress on Evolutionary Computation, 2015, 1, 773-780. https:// doi.org/10.1109/CEC.2005.1554761

4. Birattari, M., Stützle, T., Paquete, L., Varrentrapp, K. A Racing Algorithm for Configuring Metaheuristics. Proceedings of the Genetic and Evolutionary Computation Conference, 2002, 11-18.

5. Blum, C., Roli, A. Metaheuristics in Combinatorial Optimization: Overview and Conceptual Comparison. ACM Computing Surveys, 2003, 35(3), 268-308. https:// doi.org/10.1145/937503.937505

6. Civicioglu, P. Transforming Geocentric Cartesian Coordinates to Geodetic Coordinates by Using Differential Search Algorithm. Computers \& Geosciences, 2012, 46, 229-247. https://doi.org/10.1016/j.cageo.2011.12.011

7. Črepinšek, M., Liu, S.-H., Mernik, L. A Note on Teaching-Learning-Based Optimization Algorithm. Information Sciences, 2012, 212, 79-93. https://doi. org/10.1016/j.ins.2012.05.009

8. Črepinšek, M., Liu, S.-H., Mernik, M. Exploration and Exploitation in Evolutionary Algorithms: A Survey. ACM Computing Surveys, 2013, 45(3), 1-35. https://doi. org/10.1145/2480741.2480752

9. Črepinšek, M., Liu, S.-H., Mernik M. Replication and Comparison of Computational Experiments in Applied Evolutionary Computing: Common Pitfalls and Guidelines to Avoid them. Applied Soft Computing, 2014, 19, 161-170. https://doi.org/10.1016/j.asoc.2014.02.009

10. Derrac, J., García, S., Hui, S., Suganthan, P., Herrera, F. Analyzing Convergence Performance of Evolutionary Algorithms: A Statistical Approach. Information Sciences, 2014, 289, 41-58. https://doi.org/10.1016/j. ins.2014.06.009

11. Eiben, Á. E., Hinterding, R., Michalewicz, Z. Parameter Control in Evolutionary Algorithms. IEEE Transactions on Evolutionary Computation, 1999, 3(2), 124141. https://doi.org/10.1109/4235.7r71166
12. Eiben,Á. E., Jelasity, M. A Critical Note on Experimental Research Methodology in EC. Proceedings of the World on Congress on Computational Intelligence, 2002, 582587. https://doi.org/10.1109/CEC.2002.1006991

13. Friedman, M. The Use of Ranks to Avoid the Assumption of Normality Implicit in the Analysis of Variance. Journal of the American Statistical Association, 1937, 32(200), 675-701. https://doi.org/10.1080/01621459.19 37.10503522

14. Friedman, M. A Comparison of Alternative Tests of Significance for the Problem of M Rankings. The Annals of Mathematical Statistics, 1940, 11(1), 86-92. https://doi. org/10.1214/aoms/1177r731944

15. Gao, W. F., Liu, S.-Y., Huang, L.-L. Enhancing Artificial Bee Colony Algorithm Using More Information-Based Search Equations. Information Sciences, 2014, 270, 112-133. https://doi.org/10.1016/j.ins.2014.02.104

16. Glickman, M. Dynamic Paired Comparison Models with Stochastic Variances. Journal of Applied Statistics, 2001, 28(6), 673-689. https://doi. org/10.1080/02664760120059219

17. Glickman, M. Example of the Glicko-2 System. Boston University.

18. Hansen, N., Auger, A., Finck, S., Ros, R. Real-Parameter Black-Box Optimization Benchmarking 2010: Experimental Setup. Research Report RR-7215, INRIA, 2010.

19. Holm S. A Simple Sequentially Rejective Multiple Test Procedure. Scandinavian Journal of Statistics, 1979, 60-75.

20. Hooker, J. Testing Heuristics: We Have It All Wrong. Journal of Heuristics, 1995, 1(1), 33-42. https://doi. org/10.1007/BF02430364

21. Hsieh, T.-J., Hsiao, H.-F., Yeh, W.-C. Forecasting Stock Markets Using Wavelet Transforms and Recurrent Neural Networks: An Integrated System Based on Artificial Bee Colony Algorithm. Applied Soft Computing, 2011, 11(2), 2510-2525. https://doi.org/10.1016/j. asoc.2010.09.007

22. Hsu, C.-C., Chen, C.-H., Huang, K.-K., Huang, Y.-M. A Personalized Auxiliary Material Recommendation System Based on Learning Style on Facebook Applying an Artificial Bee Colony Algorithm. Computers \& Mathematics with Applications, 2012, 64(5), 1506-1513. https://doi.org/10.1016/j.camwa.2012.03.098

23. Johnson, D. A Theoretician's Guide to the Experimental Analysis of Algorithms. Data Structures, Near Neighbor Searches, and Methodology: Fifth and Sixth DIMACS Implementation Challenges, 2002, 59, 215-250. 
24. Kang, F., Li, J., Ma, Z. Rosenbrock Artificial Bee Colony Algorithm for Accurate Global Optimization of Numerical Functions. Information Sciences, 2011, 181(16), 3508-3531. https://doi.org/10.1016/j.ins.2011.04.024

25. Karaboga, D. An Idea Based on Honey Bee Swarm for Numerical Optimization. Erciyes University, 2005.

26. Karaboga, D., Basturk, B. On the Performance of Artificial Bee Colony (ABC) Algorithm. Applied Soft Computing, 2008, 8(1), 687-697. https://doi.org/10.1016/j. asoc.2007.05.007

27. Karaboga, D., Akay, B. A Comparative Study of Artificial Bee Colony Algorithm. Applied Mathematics and Computation, 2009, 214(1), 108-132. https://doi. org/10.1016/j.amc.2009.03.090

28. Karaboga, D., Akay, B. A Survey: Algorithms Simulating Bee Swarm Intelligence. Artificial Intelligence Review, 2009, 31(1-4), 61-85. https://doi.org/10.1007/s10462009-9127-4

29. Karaboga, D., Gorkemli, B., Ozturk, C., Karaboga, N. A Comprehensive Survey: Artificial Bee Colony (ABC) Algorithm and Applications. Artificial Intelligence Review, 2014, 42(1), 21-57. https://doi.org/10.1007/ s10462-012-9328-0

30. Karaboga, D., Gorkemli, B. A Quick Artificial Bee Colony (qABC) Algorithm and Its Performance on Optimization Problems. Applied Soft Computing, 2014, 23, 227238. https://doi.org/10.1016/j.asoc.2014.06.035

31. Kiran, M. S., Findik, O. A Directed Artificial Bee Colony Algorithm. Applied Soft Computing, 2015, 26, 454-462. https://doi.org/10.1016/j.asoc.2014.10.020

32. LaTorre, A., Muelas, S., Pena, J.-M. A Comprehensive Comparison of Large Scale Global Optimizers. Information Sciences, 2015, 316, 517-549. https://doi. org/10.1016/j.ins.2014.09.031

33. Lee, Y., Filliben, J., Micheals, R., Phillips, P. J. Sensitivity Analysis for Biometric Systems: A Methodology Based on Orthogonal Experiment Designs. Computer Vision and Image Understanding, 2013, 117(5), 532550. https://doi.org/10.1016/j.cviu.2013.01.003

34. Li, G., Niu, P., Xiao, X. Development and Investigation of Efficient Artificial Bee Colony Algorithm for Numerical Function Optimization. Applied Soft Computing, 2012, 12(1), 320-332. https://doi.org/10.1016/j. asoc.2011.08.040

35. Liao, T., Aydin, D., Stützle, T. Artificial Bee Colonies for Continuous Optimization: Experimental Analysis and Improvements. Swarm Intelligence, 2013, 7(4), 327356. https://doi.org/10.1007/s11721-013-0088-5
36. Liu, S.-H., Mernik, M., Bryant, B. R. To Explore or to Exploit: An Entropy-Driven Approach for Evolutionary Algorithms. International Journal of Knowledge-Based and Intelligent Engineering Systems, 2009, 13(3), 185206. https://doi.org/10.3233/KES-2009-0184

37. Liu, S.-H., Mernik, M., Hrnčič, D., Črepinšek, M. A Parameter Control Method of Evolutionary Algorithms Using Exploration and Exploitation Measures with a Practical Application for Fitting Sovova's Mass Transfer Model. Applied Soft Computing, 2013, 13(9), 37923805. https://doi.org/10.1016/j.asoc.2013.05.010

38. Ma, M., Liang, J., Guo, M., Fan, Y., Yin, Y. SAR Image Segmentation Based on Artificial Bee Colony Algorithm. Applied Soft Computing, 2011, 11(8), 5205-5214. https://doi.org/10.1016/j.asoc.2011.05.039

39. Mernik, M., Liu, S.-H., Karaboga, D., Črepinšek, M. On Clarifying Misconceptions When Comparing Variants of the Artificial Bee Colony Algorithm by Offering a New Implementation. Information Sciences, 2015, 291, 115-127. https://doi.org/10.1016/j.ins.2014.08.040

40. Nannen, V., Eiben, Á. E. Relevance Estimation and Value Calibration of Evolutionary Algorithm Parameters. IJCAI, 2007, 7, 975-980.https://doi.org/10.1109/ CEC.2007.4424460

41. Neyman, J., Pearson, E. On the Problem of the Most Efficient Tests of Statistical Hypotheses. Springer, 1992. https://doi.org/10.100r/978-1-4612-0919-5_6

42. Pellegrini, P., Mascia, F., Stutzle, T., Birattari, M., Mauro. On the Sensitivity of Reactive Tabu Search to Its Meta-Parameters. Soft Computing, 2011, 18(11), 21772190. https://doi.org/10.1007/s00500-013-1192-6

43. Piotrowski, A. Regarding the Rankings of Optimization Heuristics Based on Artificially-Constructed Benchmark Functions. Information Sciences, 2015, 297, 191201. https://doi.org/10.1016/j.ins.2014.11.023

44. Samanta, S., Chakraborty, S. Parametric Optimization of Some Non-Traditional Machining Processes Using Artificial Bee Colony Algorithm. Engineering Applications of Artificial Intelligence, 2011, 24(6), 946-95\%. https://doi.org/10.1016/j.engappai.2011.03.009

45. Smit, S. Parameter Tuning and Scientific Testing in Evolutionary Algorithms. Vrije Universiteit, 2012.

46. Ursem, R. K. Diversity-Guided Evolutionary Algorithms. Parallel Problem Solving from Nature PPSN VII, 2002, 462-471. https://doi.org/10.1007/3-54045712-r_45

47. Veček, N.,Liu, S.-H., Črepinšek, M., Mernik, M. Experimental Results. http://lpm.uni-mb.si/crepinsek/Upload.zip 
48. Veček, N., Mernik, M., Črepinšek, M. A Chess Rating System for Evolutionary Algorithms: A New Method for the Comparison and Ranking of Evolutionary Algorithms. Information Sciences, 2014, 277, 656-679. https://doi.org/10.1016/j.ins.2014.02.154

49. Veček, N., Mernik, M., Filipič, B., Črepinšek M. Parameter Tuning with Chess Rating System (CRS-Tuning) for Meta-Heuristic Algorithms. Information Sciences, 2016, 372, 446-469. https://doi.org/10.1016/j. ins.2016.08.066

50. Veček, N., Črepinšek, M., Mernik, M. On the Influence of the Number of Algorithms, Problems and Independent Runs in the Comparison of Evolutionary Algorithms. Applied Soft Computing, 2017, 54, 23-45. https://doi. org/10.1016/j.asoc.2017.01.011

51. Wilcoxon, F. Individual Comparisons by Ranking Methods. Biometrics Bulletin, 1945, 1, 80-83. https:// doi.org/10.2307/3001968

52. Xiang, W.-L., An, M.-Q. An Efficient and Robust Artificial Bee Colony Algorithm for Numerical Optimization. Computers \& Operations Research, 2013, 40(5), 12561265. https://doi.org/10.1016/j.cor.2012.12.006
53. Xu, C., Duan, H., Liu, F. Chaotic Artificial Bee Colony Approach to Uninhabited Combat Air Vehicle (UCAV) Path Planning. Aerospace Science and Technology, 2010, 14(8), 535-541. https://oi.org/10.1016/j. ast.2010.04.008

54. Yeh, W.-C., Hsieh, T.-J. Solving Reliability Redundancy Allocation Problems Using an Artificial Bee Colony Algorithm. Computers \& Operations Research, 2011, 38(11), 1465-1473. https://doi.org/10.1016/j. cor.2010.10.028

55. Zhang, C., Ouyang, D., Ning, J. An Artificial Bee Colony Approach for Clustering. Expert Systems with Applications, 2010, 37(7), 4761-4767. https://doi.org/10.1016/j. eswa.2009.11.003

56. Zhang, R., Song, S., Wu, C. A Hybrid Artificial Bee Colony Algorithm for the Job Shop Scheduling Problem. International Journal of Production Economics, 2013, 141(1), 167-178. https://doi.org/10.1016/j. ijpe.2012.03.035

57. Zhang, W., Wang, N., Yang, S. Hybrid Artificial Bee Colony Algorithm for Parameter Estimation of Proton Exchange Membrane Fuel Cell. International Journal of Hydrogen Energy, 2013, 38(14), 5796-5806. https://doi. org/10.1016/j.ijhydene.2013.01.058

\section{Summary / Santrauka}

Artificial Bee Colony (ABC) is a successful meta-heuristic algorithm that has been greatly utilised by researchers. Through our practical experience of $A B C$, we have noticed that the recommended formula 'limit' = ne * D may not be the best choice for different problems. In this work, a set of experiments using horizontal and vertical approaches has been designed and executed with the aim of observing the effect of 'limit' on ABC. The results have been statistical analysed using Null Hypothesis Significance Testing (NHST) as well as the Chess Rating System for Evolutionary Algorithms (CRS4EAs), which is a novel approach for comparing meta-heuristic algorithms. It is shown that the recommended formula is not the best setting for different problems and approaches. Hence, the control parameter 'limit' should be tuned or controlled. The other important result of this study is to show that CRS4EAs is comparable but also shows benefits over NHST.

Dirbtinė bičių kolonija (ABC) yra sėkmingas, mokslininkų plačiai naudojamas metaeuristinis algoritmas. Per savo praktinę $\mathrm{ABC}$ patirtį straipsnio autoriai pastebejo, kad rekomenduojama formulè 'limit' = ne * D ne visuomet yra geriausias pasirinkimas tam tikroms problemoms spręsti. Su tikslu įvertinti formulès elemento 'limit' poveikį $A B C$, straipsnio autoriai sukūrè ir atliko eksperimentus, paremtus horizontaliais ir vertikaliais metodais. Gauti rezultatai statistiškai analizuoti naudojant hipotezès reikšmingumo testavimą (NHST) bei Šachmatų reitingų sistemą Evoliucijos algoritmui (CRS4EAs). Tai yra naujas metodas metaeuristiniams algoritmams palyginti. Straipsnyje įrodoma, kad rekomenduojama formulè išties nẻra geriausias skirtingų problemų ir metodų nustatymas. Taigi, kontrolès parametras 'limit' turètų būti nustatytas arba kontroliuojamas. Kitas svarbus šio tyrimo rezultatas - parodoma, kad CRS4EAs yra palyginamas, tačiau, palyginus su NHST, yra pranašesnis. 